\title{
Homochiral 2D Porous Covalent Organic Frameworks for Heterogeneous Asymmetric Catalysis
}

Xiuren Wang, ${ }^{\dagger}$ Xing Han,${ }^{\dagger}$ Jie Zhang, ${ }^{\dagger}$ Xiaowei Wu, ${ }^{\dagger}$ Yan $\mathrm{Liu}^{*},{ }^{\dagger}$ and Yong Cui ${ }^{*}, \dagger^{\dagger}$,

'School of Chemistry and Chemical Engineering and State Key Laboratory of Metal Matrix Composites, Shanghai Jiao Tong University, Shanghai 200240, China.

${ }^{*}$ Collaborative Innovation Center of Chemical Science and Engineering, Tianjin 300072, China.

Email: 1iuy@sjtu.edu.cn, yongcui@sjitu.edu.cn

\section{Table of Content}

1. Materials and general procedures

2. Synthesis of CCOFs

3. General procedure for asymmetric catalysis

4. Tables S1 Comparison with different heterogeneous catalysts in the reaction

5. Figure S1 FT-IR spectra

6. Figure $\mathbf{S 2}$ NMR spectra of the CCOFs and monomers

7. Figure S3 ESI-MS spectra

8. Figure $\mathbf{S 4}$ The Solid-CD spectra

9. Figure $\mathbf{S 5}$ TGA curves

10. Figure S6 SEM and TEM

11. Figures S7\&S8 PXRD patterns

12. Figure S9 BET plots

13. Figures S10-S13 Structural modeling and PXRD analysis of COFs

14. Tables S2\&S3 Fractional atomic coordinates for the unit cell of COFs

15. Figure S14 Spectra data of the catalysis

16. Figure S15 Calculated space filling model of different Aromatic Aldehydes 


\section{Materials and general procedures}

General. All the chemicals are commercial available, and used without further purification. All solvents were dried and distilled according to conventional methods. The IR (KBr pellet) spectra were recorded $\left(400-4000 \mathrm{~cm}^{-1}\right.$ region) on a Nicolet Magna 750 FT-IR spectrometer. The CD spectra were recorded on a J-800 spectropolarimeter (Jasco, Japan). Powder X-ray diffraction data (PXRD) were collected on a DMAX2500 diffractometer using $\mathrm{Cu} \mathrm{K} \alpha$ radiation. Thermogravimetric analyses (TGA) were carried out in an air atmosphere with a heating rate of $20{ }^{\circ} \mathrm{C} / \mathrm{min}$ on a STA449C integration thermal analyzer. SEM was conducted on a JEOL JSM-7401F electron microscope. ${ }^{1} \mathrm{H}$ and ${ }^{13} \mathrm{CNMR}$ experiments were carried out on a MERCURY plus 400 spectrometer operating at resonance frequencies of $400 \mathrm{MHz}$. Analytical high performance liquid chromatography (HPLC) was performed on YL-9100 HPLC with UV detection at $220 \mathrm{~nm}$. Analytical CHIRALCEL OD-H and AD-H columns $(4.6 \mathrm{~mm} \times 25 \mathrm{~cm})$ from Daicel were used. Absolute stereochemistry was assigned by comparison of optical rotation with reported values. The porous properties of the covalent organic frameworks (COFs) were investigated by nitrogen adsorption and desorption at 77.3 K using ASAP 2020 V4.00 (V4.00 H) Serial \#: 1674 from USA. Pore size distributions and pore volumes were derived from the adsorption isotherms. Samples were pretreated by the supercritical carbon dioxide and degassed at $100{ }^{\circ} \mathrm{C}$ for $4 \mathrm{~h}$ under high vacuum before analysis. The theoretical surface area was calculated by Atom Volumes \&Surface (Materials Studio, version 7.0). 


\section{Synthesis of the CCOFs}

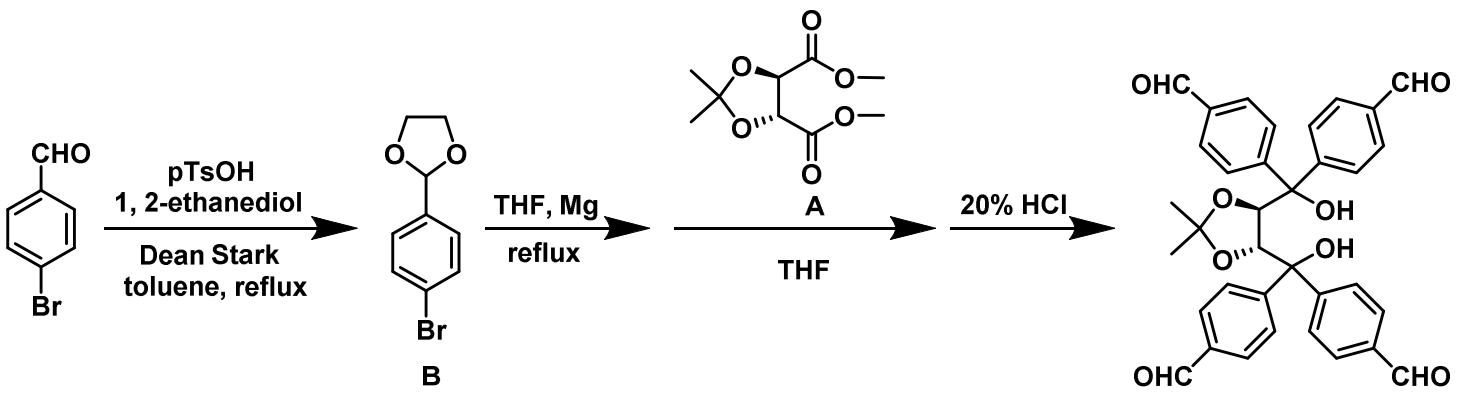

$(R, R)$-TTA

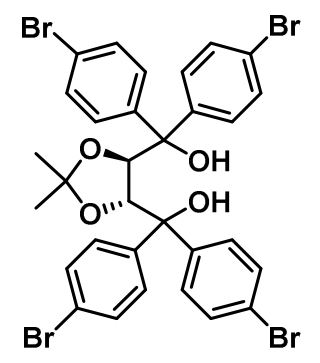

$(R, R)-\mathrm{C}$
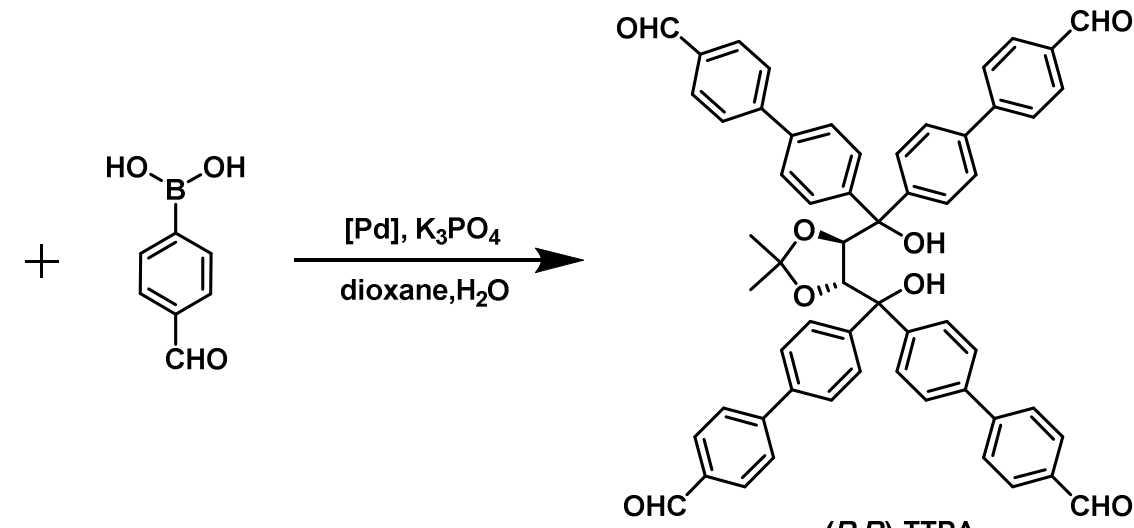

$(R, R)$-C was synthesized according to the published procedure (Wang, X.; Zhang, J.; Liu, Y and Cui, Y. Bull. Chem. Soc. Jpn. 2014, 87, 435).

2.1 Synthesis of $(\boldsymbol{R}, \boldsymbol{R})$-TTA: Magnesium $(1.08 \mathrm{~g}, 45 \mathrm{mmol})$, anhydrous THF (30 $\mathrm{mL}$ ), and a grain of iodine were added to a flame-dried three-necked flask. B (9.16 g, $40 \mathrm{mmol})$ in THF $(80 \mathrm{~mL})$ was then added dropwise to the solution at a rate sufficient to maintain a vigorous reflux. After that, the mixture was stirred and heated at $50{ }^{\circ} \mathrm{C}$ for another $2 \mathrm{~h}$. A (1.46 g, $6.7 \mathrm{mmol})$ in THF $(50 \mathrm{~mL})$ was added dropwise to the solution at $0{ }^{\circ} \mathrm{C}$ over $1 \mathrm{~h}$, and then the reaction mixture was kept for an additional $12 \mathrm{~h}$ at room temperature. The saturated $10 \% \mathrm{HCl}$ solution was added carefully to quench the reaction until the $\mathrm{pH}$ value is reached to 2 at $0{ }^{\circ} \mathrm{C}$. and then the reaction mixture was stirred for an additional $24 \mathrm{~h}$ at room temperature. The organic layer was separated, and the aqueous phase was extracted with diethyl ether $(3 \times 50 \mathrm{~mL})$. The combined organic phase was then dried over $\mathrm{Na}_{2} \mathrm{SO}_{4}$ and concentrated. The crude material was purified by flash chromatography on silica gel (hexane: ethyl acetate = $60: 40)$ to give product $\left(1.98 \mathrm{~g}, 51 \%\right.$ yield) as a yellow solid. ${ }^{1} \mathrm{H}$ NMR (400 MHz, $\left.\mathrm{CDCl}_{3}\right) \delta 9.91(\mathrm{~s}, 2 \mathrm{H}), 9.85(\mathrm{~s}, 2 \mathrm{H}), 7.83(\mathrm{~d}, J=8.5 \mathrm{~Hz}, 4 \mathrm{H}), 7.76-7.69(\mathrm{~m}, 8 \mathrm{H})$, 
$7.50(\mathrm{~d}, J=8.4 \mathrm{~Hz}, 4 \mathrm{H}), 5.53(\mathrm{~s}, 2 \mathrm{H}), 4.49(\mathrm{~s}, 2 \mathrm{H}), 1.13(\mathrm{~s}, 6 \mathrm{H}) .{ }^{13} \mathrm{C} \mathrm{NMR}(101 \mathrm{MHz}$, $\left.\mathrm{CDCl}_{3}\right) \delta 192.2,191.9,151.0,148.6,135.3,135.3,129.5,129.0,128.8,128.2,109.8$, 80.8, 77.7, 27.0. FTIR (KBr pellet, v/cm ${ }^{-1}$ ): 3329 (s), 2986 (w), 2922 (w), 1912 (w), 1702 (s), 1606 (s), 1575 (m), 1418 (w), 1383 (m), 1308 (m), 1240 (w), 1214 (s), 1174 (m), $1064(\mathrm{~m}), 1014(\mathrm{w}), 886(\mathrm{w}), 845(\mathrm{~m}), 817(\mathrm{~s}), 762(\mathrm{~m}), 668(\mathrm{w}), 517(\mathrm{w})$. ESI-MS m/z: 577.19 (Calcd m/z 577.19 for [M-H]').

2.2 Synthesis of $(\boldsymbol{R}, \boldsymbol{R})$-TTPA: $(R, R)-\mathrm{C}(1.56 \mathrm{~g}, 2.0 \mathrm{mmol})$, (4-formylphenyl)boronic acid $(1.50 \mathrm{~g}, 10.0 \mathrm{mmol}), \mathrm{K}_{3} \mathrm{PO}_{4}(6.4 \mathrm{~g}, 24 \mathrm{mmol})$ and $\mathrm{Pd}\left(\mathrm{P}(\mathrm{Ph})_{3}\right)_{4}(230 \mathrm{mg}, 0.2$ $\mathrm{mmol})$ in dioxane $/ \mathrm{H}_{2} \mathrm{O}(3 / 1 \mathrm{v} / \mathrm{v}, 24 \mathrm{~mL})$ were degassed for $10 \mathrm{~min}$. the suspension was stirred under $\mathrm{N}_{2}$ at $100{ }^{\circ} \mathrm{C}$ for $24 \mathrm{~h}$. After cooling to room temperature, the mixture was concentrated and extracted with EtOAc $/ \mathrm{H}_{2} \mathrm{O}$. The organic phase was dried over anhydrous $\mathrm{Na}_{2} \mathrm{SO}_{4}$ and then concentrated under reduced pressure to remove the solvent. The crude product was purified by silica gel column chromatography (hexanes/ethyl acetate $(4: 3 \mathrm{v} / \mathrm{v})$ to afford $(R, R)$-c. Yield: $(742.6 \mathrm{mg}$, 42\%). ${ }^{1} \mathrm{H}$ NMR (400 MHz, $\left.\mathrm{CDCl}_{3}\right) \delta 10.03$ (d, $\left.J=4.4 \mathrm{~Hz}, 2 \mathrm{H}\right), 10.00$ (d, $J=4.0 \mathrm{~Hz}$, 2H), $7.96(\mathrm{dd}, J=8.2,2.0 \mathrm{~Hz}, 4 \mathrm{H}), 7.89(\mathrm{dd}, J=8.2,2.4 \mathrm{~Hz}, 4 \mathrm{H}), 7.80(\mathrm{~d}, J=7.6 \mathrm{~Hz}$, 4H), 7.74 (d, $J=8.4 \mathrm{~Hz}, 4 \mathrm{H}), 7.67(\mathrm{~m}, 8 \mathrm{H}), 7.52$ (q, $J=8.8 \mathrm{~Hz}, 8 \mathrm{H}), 4.71$ (d, $J=2.4$ $\mathrm{Hz}, 2 \mathrm{H}), 4.55$ (s, 2H), 1.22 (s, 6H). $\left.{ }^{13} \mathrm{C} \mathrm{NMR} \mathrm{(101} \mathrm{MHz,} \mathrm{CDCl}_{3}\right) \delta$ 192.0, 191.9, $146.5,146.4,145.7,142.9,138.7,138.3,135.0,130.3,130.2,129.2,128.3,127.4$, 127.4, 127.0, 126.2, 109.5, 81.1, 77.7, 27.2. FTIR (KBr pellet, $\left.v / \mathrm{cm}^{-1}\right): 3348(\mathrm{~m})$, 3025 (w), 2985 (w), 2885 (w), 1911 (w), 1702 (s), 1605 (s), 1577 (w), 1495 (w), 1384 (w), $1310(\mathrm{w}), 1214$ (s) , $1171(\mathrm{~m}), 1061(\mathrm{~m}), 1006(\mathrm{w}), 842$ (m), 816 (s), $776(\mathrm{~m}), 664$ (w), $523(w)$. ESI-MS m/z: 881.31 (Calcd m/z 881.32 for [M-H]).

2.3 Synthesis of CCOF-1: $25 \mathrm{~mL}$ schlenk was charged with $(R, R)$-TTA (145 mg, $0.25 \mathrm{mmol}$ ) and 4,4'-diaminodiphenylmethane (4,4'-DADPM) (99 $\mathrm{mg}, 0.5 \mathrm{mmol}$ ), anhydrous 1, 4-dioxane $(6 \mathrm{~mL})$. The resulting mixture was sonicated for a few minutes until the solid was completely dissolved. The solution was then added $9 \mathrm{M}$ acetic acid $(1.5 \mathrm{~mL})$, and the Schlenk was flash frozen at $77 \mathrm{~K}$ using the liquid nitrogen bath, evacuated and sealed by Teflon valve. Upon warming to room temperature, the Schlenk was placed in an oven and heated at $100{ }^{\circ} \mathrm{C}$ for 3 days. After heating at $120{ }^{\circ} \mathrm{C}$ for $72 \mathrm{~h}$, gray solid at the bottom of the tube was isolated by hot filtration and washed with anhydrous 1, 4-dioxane. The powder collected was then solvent exchanged with THF for three times and acetone for one time and then dried 
at $100{ }^{\circ} \mathrm{C}$ under vacuum for $24 \mathrm{~h}$ to give a gray powder $(158 \mathrm{mg}, 70 \%$ yield $)$. FTIR (KBr pellet, v/cm-1): 3329 (s), 3027 (w), 2981 (w), 2296 (w), 1909 (w), 1699 (w), 1624 (s), 1602 (m), 1565 (w), 1506 (s), 1410 (w), 1377 (w), 1309 (w), 1199 (m), 1171 (m), $1057(\mathrm{~s}), 1016(\mathrm{~m}), 974(\mathrm{w}), 887(\mathrm{~m}), 832(\mathrm{~s}), 764(\mathrm{~m}), 714(\mathrm{w}), 589(\mathrm{w})$.

2.4 Synthesis of CCOF-2: This COF was synthesized following the similar method as described about CCOF-1 by using $(R, R)$-TTPA (132 $\mathrm{mg}, 0.15 \mathrm{mmol})$ and 4,4'-diaminodiphenylmethane (4,4'-DADPM) (59.5mg, $0.3 \mathrm{mmol})$. The powder collected was then solvent exchanged with THF for three times and acetone for one time and then dried at $100{ }^{\circ} \mathrm{C}$ under vacuum for $24 \mathrm{~h}$ to give a gray powder $(116 \mathrm{mg}$, 64\% yield). FTIR (KBr pellet, v/cm ${ }^{-1}$ ): $3346(\mathrm{~m}), 3026(\mathrm{w}), 2980(\mathrm{w}), 2886(\mathrm{w})$, $1913(\mathrm{w}), 1700$ (w), 1623 (m), 1600 (m), 1501 (s), 1373 (w), 1309 (w), 1245 (w), 1199 (w), 1172 (m), 1057 (w), 1006 (m), 915 (w), 887 (m), 823 (s), 777 (w), 731 (w), $549(\mathrm{w})$.

\subsection{Activation of the CCOF}

Standard activation: The powder collected was then solvent exchanged with THF for three times and anhydrous acetone for one time and then dried at $100{ }^{\circ} \mathrm{C}$ under vacuum for $24 \mathrm{~h}$.

Activation for BET measurement: The COF powder was immersed in anhydrous THF, and the solvent was exchanged with fresh THF for three times and anhydrous acetone for one time. The wet sample was then transfer to a super critical drier, in which the sample was washed with five times of liquid $\mathrm{CO}_{2}$, and exchanged with fresh $\mathrm{CO}_{2}$ for 5 times with the interval of half hour. The system was heat up to $45^{\circ} \mathrm{C}$ to bring about the supercritical state of the $\mathrm{CO}_{2}$, which was bleed after half hour in very slow flow rate to ambient pressure. The sample was then transferred to vacuum chamber and evacuated to $20 \mathrm{mTorr} 100^{\circ} \mathrm{C}$ for $4 \mathrm{~h}$, yielding white powder.

\section{General procedure for asymmetric catalysis}

3.1 The diethylzinc addition reactions catalyzed by CCOF/Ti: The catalytic process was similar, here, take the procedure of CCOF-1/Ti catalyzed reactions as an example. To a dry sample of CCOF-1 $(18.1 \mathrm{mg}, 0.02 \mathrm{mmol})$ in $1 \mathrm{~mL}$ of anhydrous toluene was added $\mathrm{Ti}\left(\mathrm{O}^{i} \mathrm{Pr}\right)_{4}(85 \mathrm{mg}, 0.3 \mathrm{mmol})$, and the resulting mixture was stirred for $1 \mathrm{~h}$. The solvent and excess $\mathrm{Ti}\left(\mathrm{O}^{i} \mathrm{Pr}\right)_{4}$ were evaporated off under high vacuum. The 
residue was degassed and benzaldehyde $(10.6 \mathrm{mg}, 0.1 \mathrm{mmol})$ in toluene $(1.0 \mathrm{~mL})$ was added. The reaction mixture was cooled to $-30{ }^{\circ} \mathrm{C}$ under $\mathrm{N}_{2}$ atmosphere, diethylzinc in hexane $(200 \mu \mathrm{L}, 1.0 \mathrm{M}, 0.2 \mathrm{mmol})$ were then added to the vigorous stirring suspension and allowed to react for $10 \mathrm{~h}$ at $-30{ }^{\circ} \mathrm{C}$. The effect of the stirring is order to react uniformly. The reaction mixture was quenched by the addition of $1 \mathrm{~mL}$ of saturated ammonium chloride and $1 \mathrm{~mL}$ of diethyl ether. The organic layer was analyzed by ${ }^{1} \mathrm{H}$ NMR and HPLC to give conversion and ee values.

Notably, the use of excess amount (3 quiv.) of $\mathrm{Ti}(\mathrm{OiPr})_{4}$ to modify the CCOFs is to make sure Taddol units in the framework react completely with Ti(IV) ions for achieving high activity. Decreasing the amount of $\mathrm{Ti}(\mathrm{OiPr})_{4}$ to, for example, 2.5 equivalent led to the conversion of benzylaldehyde decreasing from 99 to $90 \%$ under otherwise identical conditions.

3.2 The diethylzinc addition reactions catalyzed by TADDOL/Ti: The procedure was the same as the above catalytic experiments.

Notably, the asymmetric addition of dialkyl zinc to aldehydes have been well studied in homogeneous catalysis, See: (a) Cozzi, P. G.; Kotrusz, P. J. Am. Chem. Soc. 2006, 128, 4940. (b) García, C.; LaRochelle, L. K.; Walsh, P. J. J. Am. Chem. Soc. 2002, 124, 10970. (c) Wieland, L. C.; Deng, H.; Snapper, M. L.; Hoveyda, A. H. J. Am. Chem. Soc. 2005, 127, 15453. (d) Balsells, J.; Davis, T. J.; Carroll, P.; Walsh, P. J. J. Am. Chem. Soc. 2002, 124, 10336. (e) Qiu, J.; Guo, C.; Zhang, X. J. Org. Chem. 1997, 62, 2665.

3.3 Recycling Experiments: The catalytic process was the same as the above catalytic experiments. After the reaction, the solution was removed by centrifugation quikly, the polymer was rinsed quickly with anhydrous toluene and isolated by centrifugation, then $\mathrm{Ti}\left(\mathrm{O}^{i} \mathrm{Pr}\right)_{4}(80 \mathrm{mg}, 0.28 \mathrm{mmol})$ were added and stirred for $1.0 \mathrm{~h}$ under $\mathrm{N}_{2}$. Benzaldehyde $(10.6 \mathrm{mg}, 0.1 \mathrm{mmol})$ in toluene $(1.0 \mathrm{~mL})$ were added, followed by diethylzinc in hexane $(200 \mu \mathrm{L}, 1.0 \mathrm{M}, 0.2 \mathrm{mmol})$ at $-30{ }^{\circ} \mathrm{C}$ under $\mathrm{N}_{2}$. Then the reaction mixture was stirred at $-30{ }^{\circ} \mathrm{C}$ for a new run. 
4. Table S1. Comparison with different heterogeneous catalysts in the addition of $\mathrm{Et}_{2} \mathrm{Zn}$ to aromatic aldehydes.

\begin{tabular}{|c|c|c|c|c|}
\hline entry & Cat & $\mathrm{Ar}$ & conv $(\%)$ & ee $(\%)$ \\
\hline 1 & CCOF-1 & $\mathrm{C}_{6} \mathrm{H}_{5}$ & 99 & 90 \\
\hline 2 & CCOF-2 & & 99 & 85 \\
\hline 3 & Cd-Binol-MOFa & & 99 & 83 \\
\hline 4 & Cu-Binol-MOFb & & 99 & 83 \\
\hline 5 & TADDOL-POP-1 ${ }^{\mathrm{C}}$ & & 99 & 90 \\
\hline 6 & TADDOL-POP-2 ${ }^{\mathrm{d}}$ & & 99 & 91 \\
\hline 7 & CCOF-1 & $p-\mathrm{MeC}_{6} \mathrm{H}_{4}$ & 99 & 93 \\
\hline 8 & CCOF-2 & & 99 & 88 \\
\hline 9 & TADDOL-POP-1 ${ }^{\mathrm{C}}$ & & 99 & 90 \\
\hline 10 & TADDOL-POP-2 $^{\mathrm{d}}$ & & 99 & 90 \\
\hline 11 & CCOF-1 & $p-\mathrm{ClC}_{6} \mathrm{H}_{5}$ & 94 & 90 \\
\hline 12 & CCOF-2 & & 96 & 86 \\
\hline 13 & Cd-Binol-MOFa & & 99 & 80 \\
\hline 14 & Cu-Binol-MOF & & 99 & 80 \\
\hline 15 & TADDOL-POP-1 ${ }^{\mathrm{C}}$ & & 99 & 85 \\
\hline 16 & TADDOL-POP-2 $^{\mathrm{d}}$ & & 99 & 88 \\
\hline 17 & CCOF-1 & $\mathrm{C}_{6} \mathrm{H}_{5} \mathrm{C}=\mathrm{CH}$ & 99 & 91 \\
\hline 18 & CCOF-2 & & 99 & 85 \\
\hline 19 & TADDOL-POP-1 ${ }^{\mathrm{C}}$ & & 99 & 46 \\
\hline 20 & CCOF-1 & 2-naphthyl & 96 & 94 \\
\hline 21 & CCOF-2 & & 96 & 95 \\
\hline 22 & TADDOL-POP-1c & & 99 & 94 \\
\hline 23 & TADDOL-POP-2 ${ }^{d}$ & & 99 & 94 \\
\hline
\end{tabular}

(a) Wu, C.; Hu, A.; Zhang, L.; Lin, W. J. Am. Chem. Soc. 2005, 127, 8940. (b) Ma, L.; Falkowski, J. M.; Abney, C.; Lin, W. Nat. Chem. 2010, 2, 838. (c) Wang, X.; Zhang, J.; Liu, Y.; Cui, Y. Bull. Chem. Soc. Jpn. 2014, 87, 435. (d) An, W.; Han, M.; Wang, C.; Yu, S.; Zhang Y.; Bai, S.; Wang, W. Chem. Eur. J. 2014, 20, 11019. 
5. Figure S1. FT-IR spectra for a) CCOF-1 and b) CCOF-2 .

a)

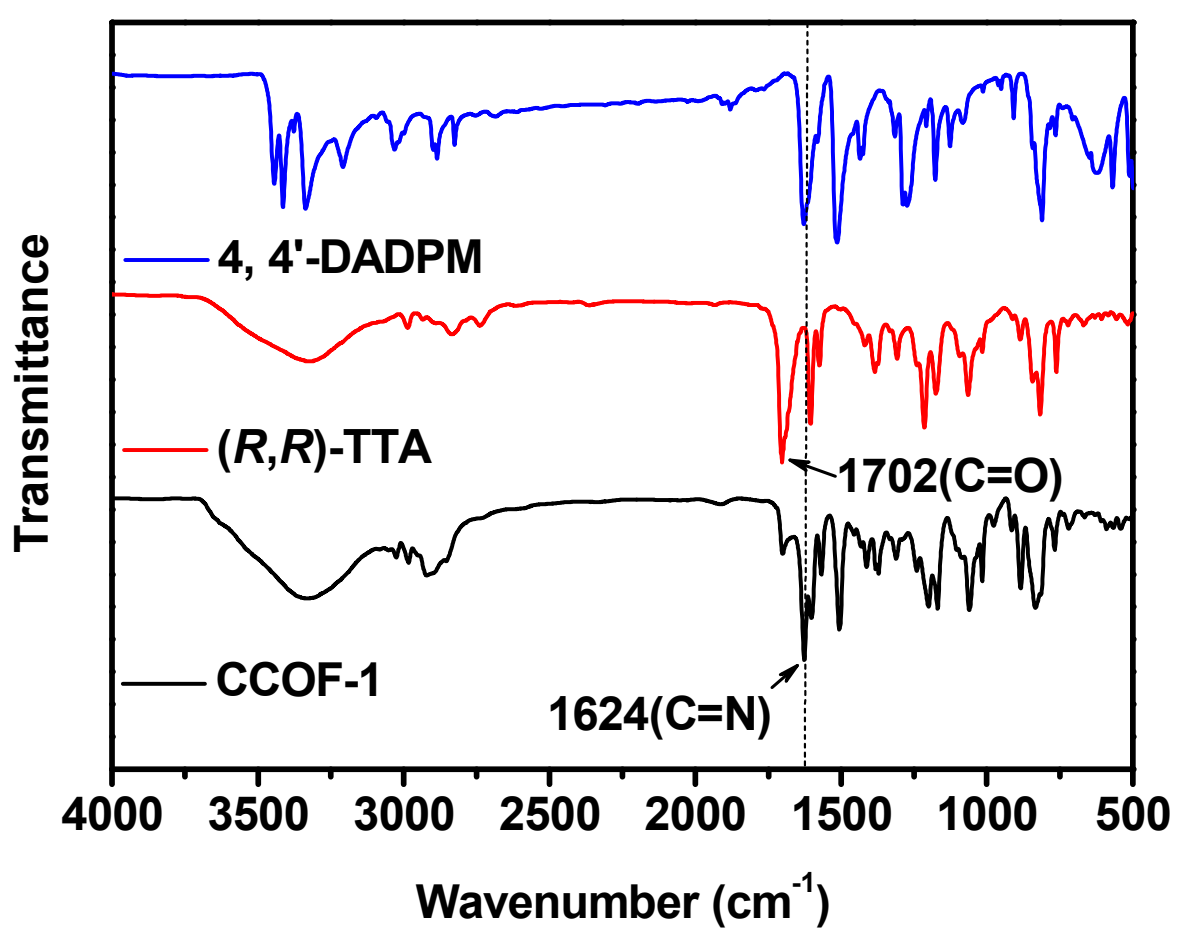

b)

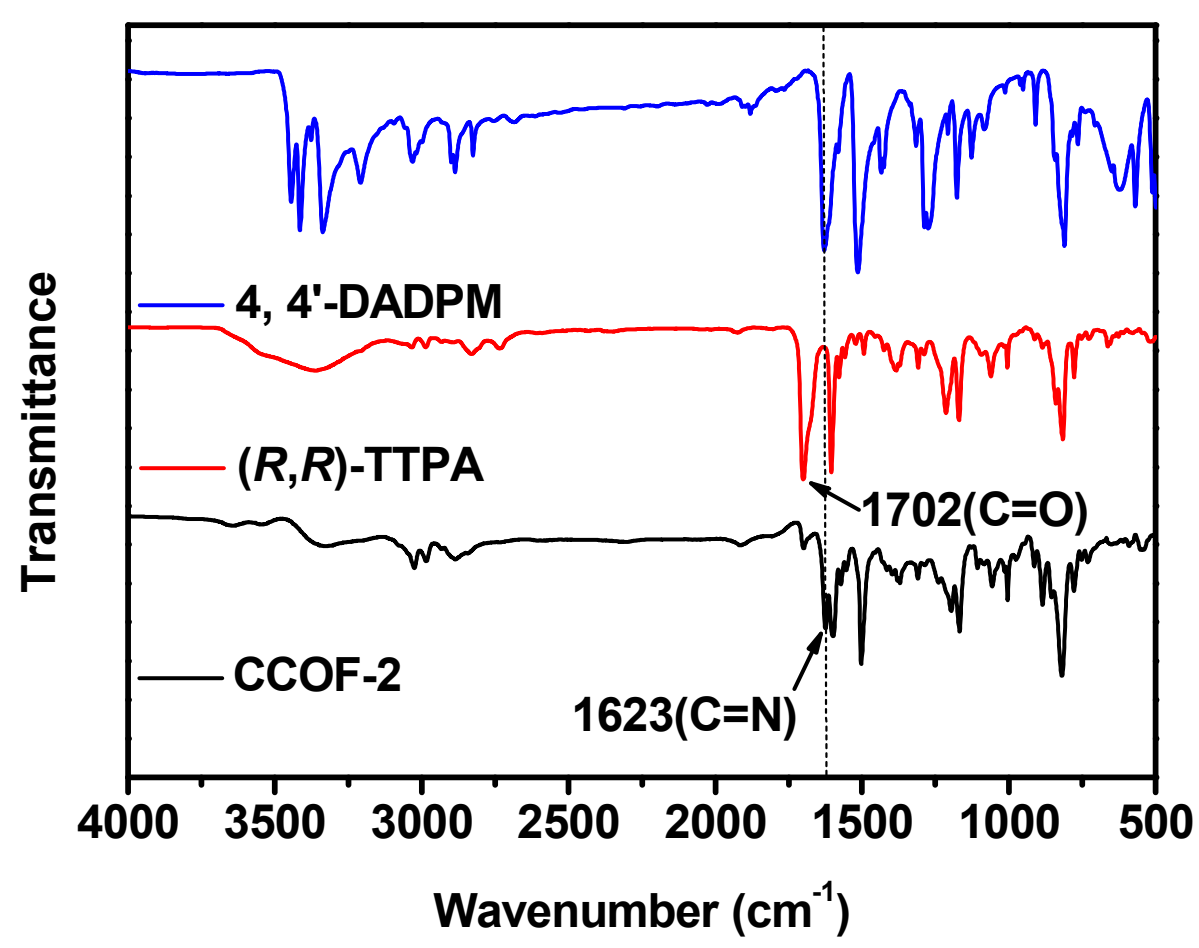


6. Figure S2. NMR spectra of the CCOFs and related monomers

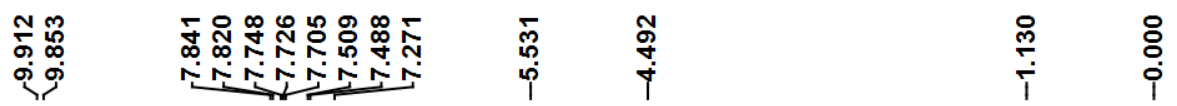<smiles>CC1(C)OC(C(O)(c2ccc(C=O)cc2)C(O)(c2ccc(C=O)cc2)c2ccc(C=O)cc2)C(O)C1c1ccc(C=O)cc1</smiles>

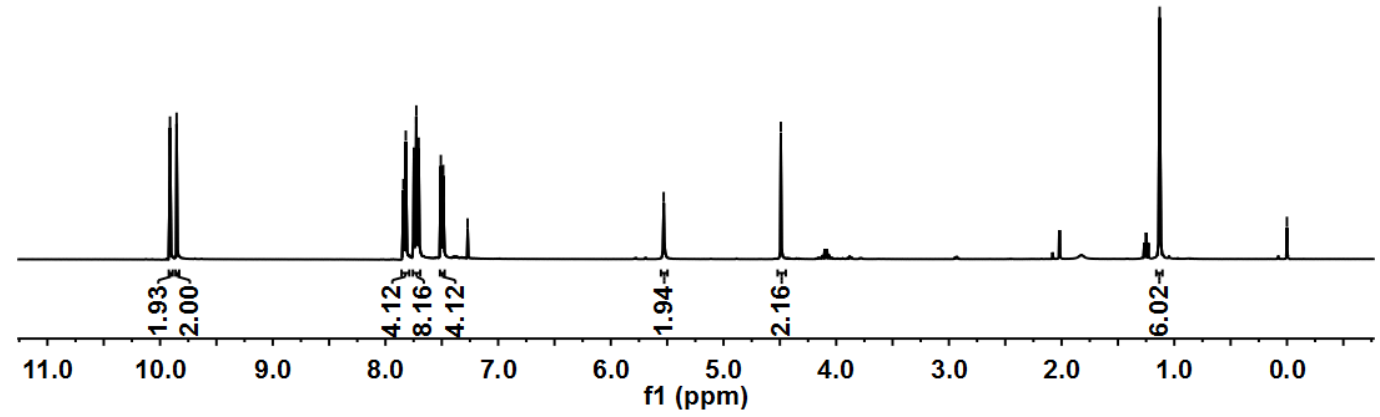

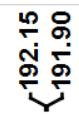

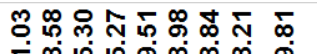

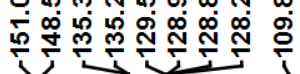

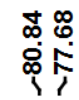

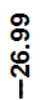<smiles>CC1(C)CC(O)C(C(O)(c2ccc(C=O)cc2)C(O)(c2ccc(C=O)cc2)c2ccc(C=O)cc2)O1</smiles>

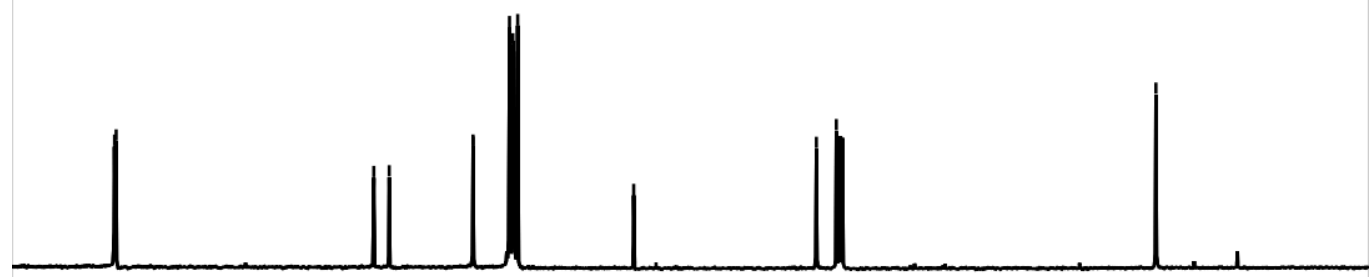

190 130 $110 \quad 90$ 


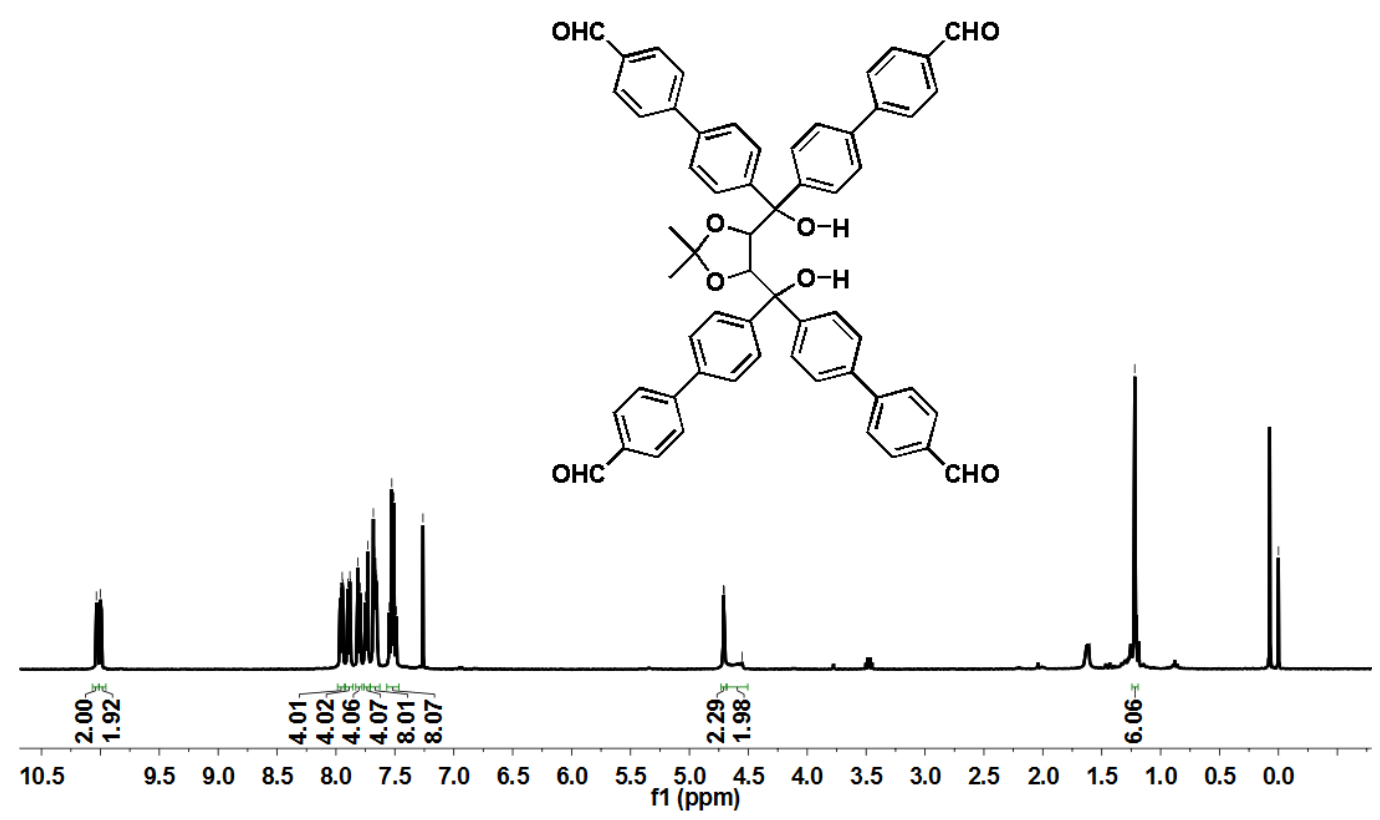

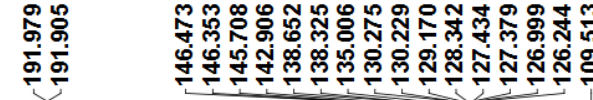

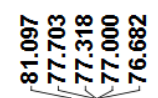

$\mathrm{OHC}$<smiles>Cc1ccc(-c2ccc(C(O)(c3ccc(-c4ccc(C=O)cc4)cc3)C3OC(C)(C)OC3CO)cc2)cc1</smiles>

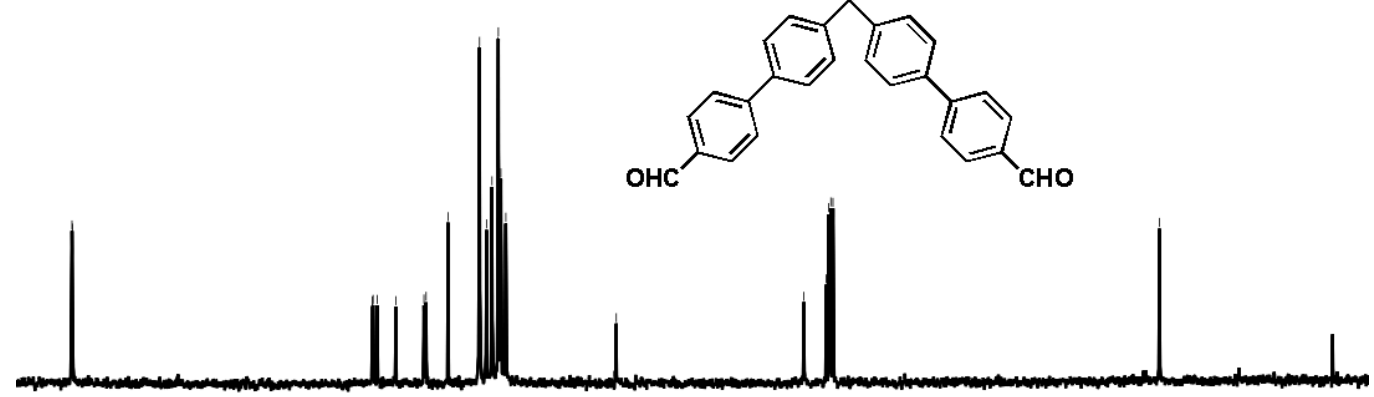

$\begin{array}{lllllllllllllllllllll}\mathrm{J} 0 & 190 & 180 & 170 & 160 & 150 & 140 & 130 & 120 & 110 \begin{array}{c}100 \\ \mathrm{f} 1(\mathrm{ppm})\end{array} & 90 & 80 & 70 & 60 & 50 & 40 & 30 & 20 & 10 & 0\end{array}$ 

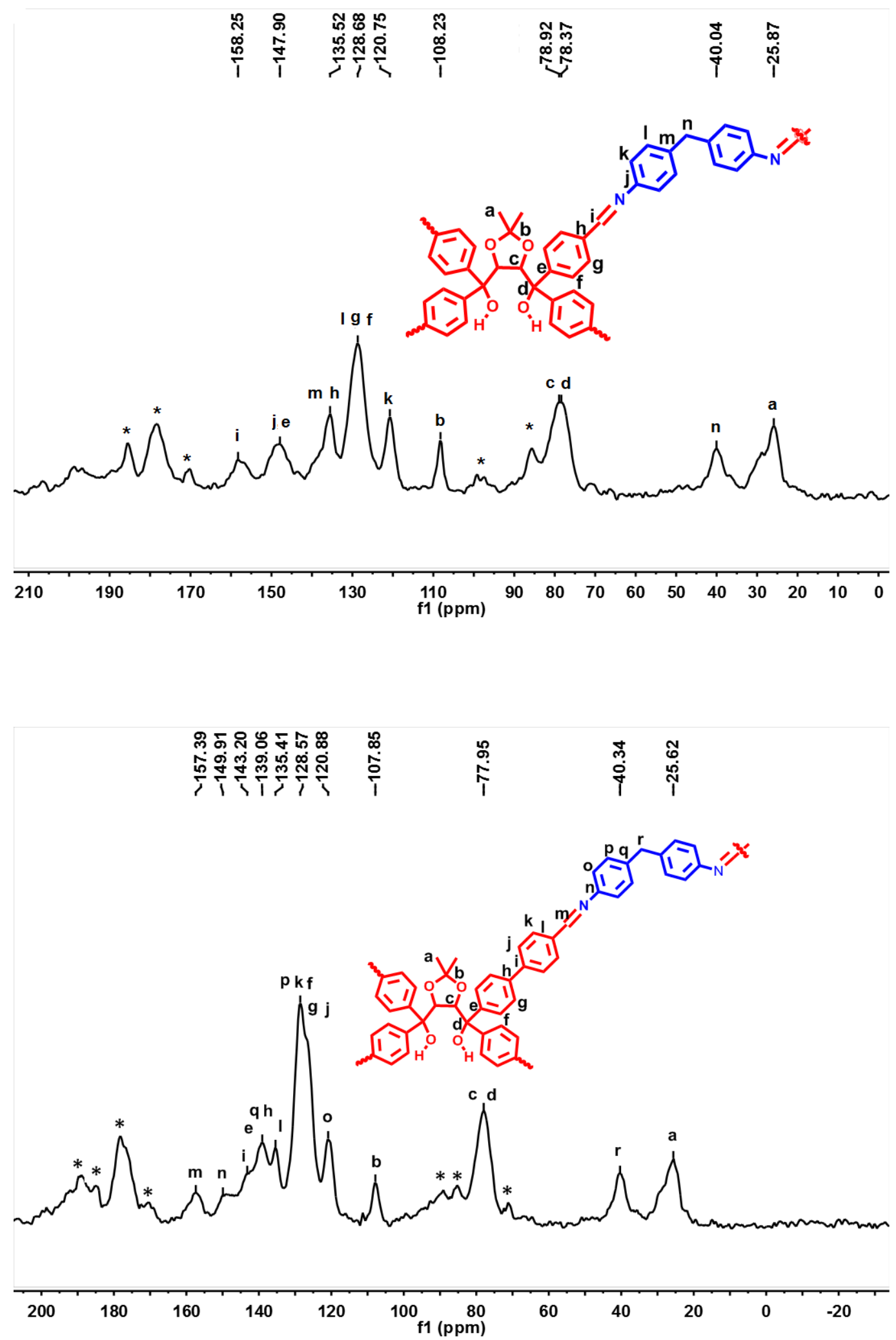


\section{Figure S3. ESI-MS spectra of the TADDOL-derived tetraaldehydes}
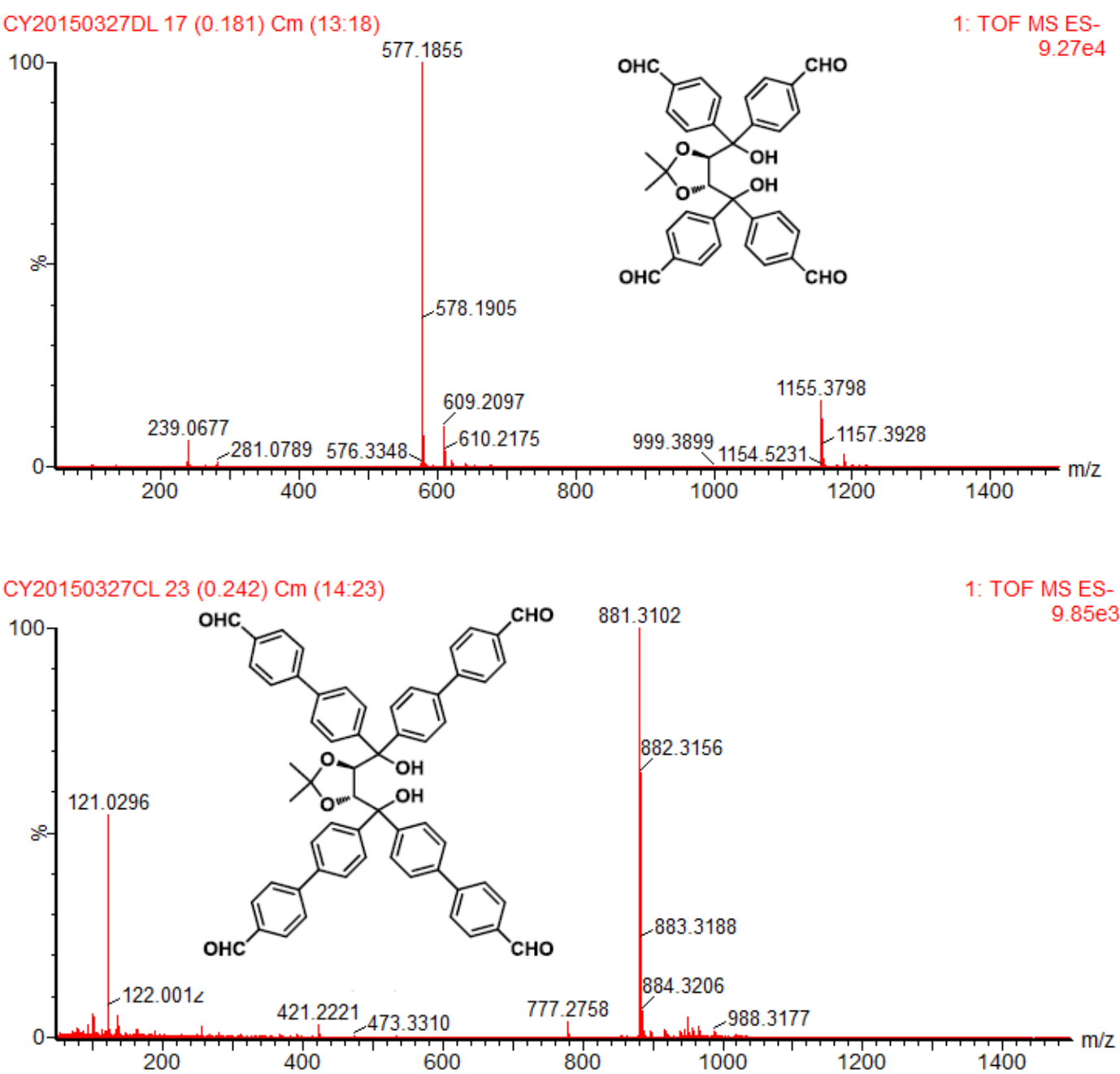

8. Figure S4. The solid-state CD spectra of the CCOFs.

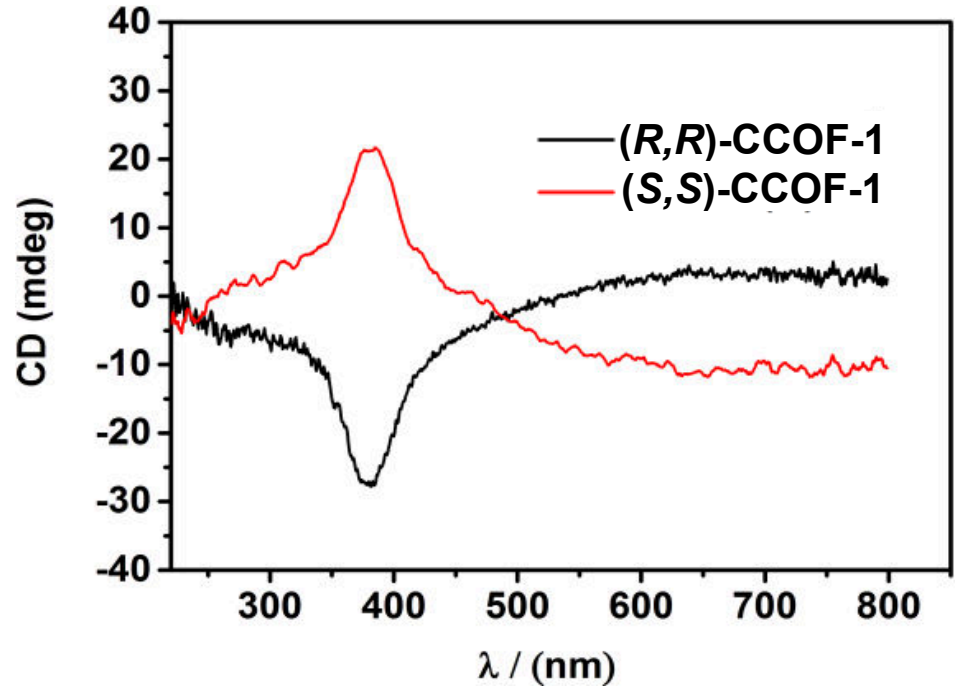




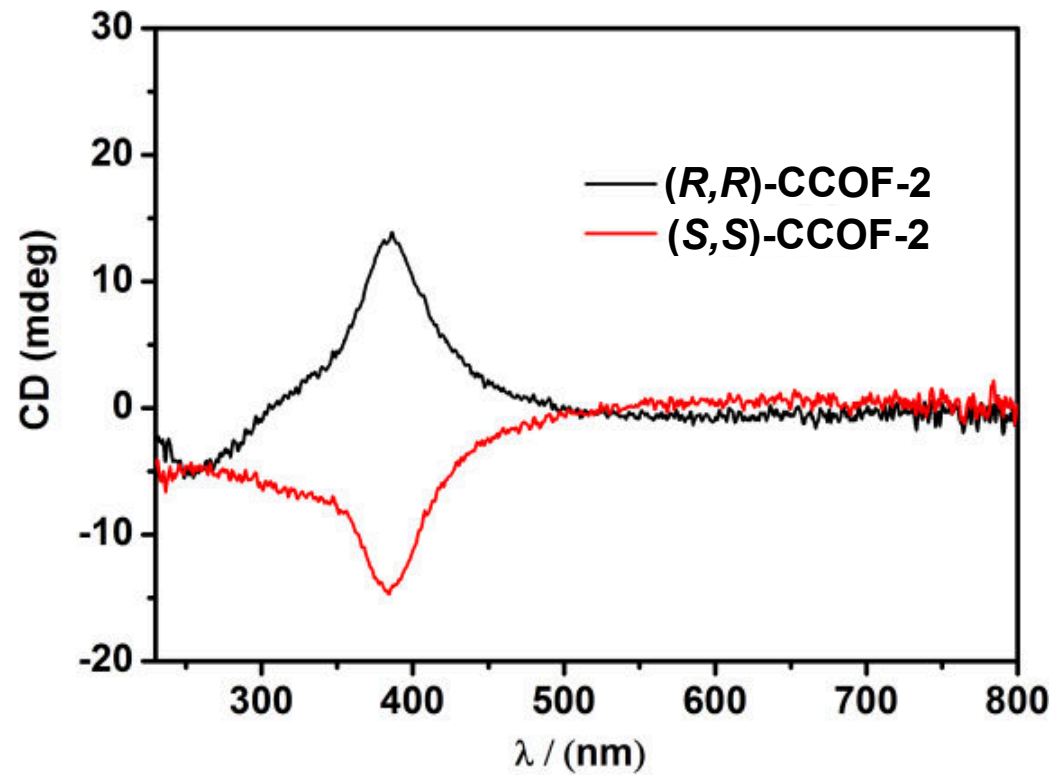

9. Figure S5. TGA curves of the CCOFs
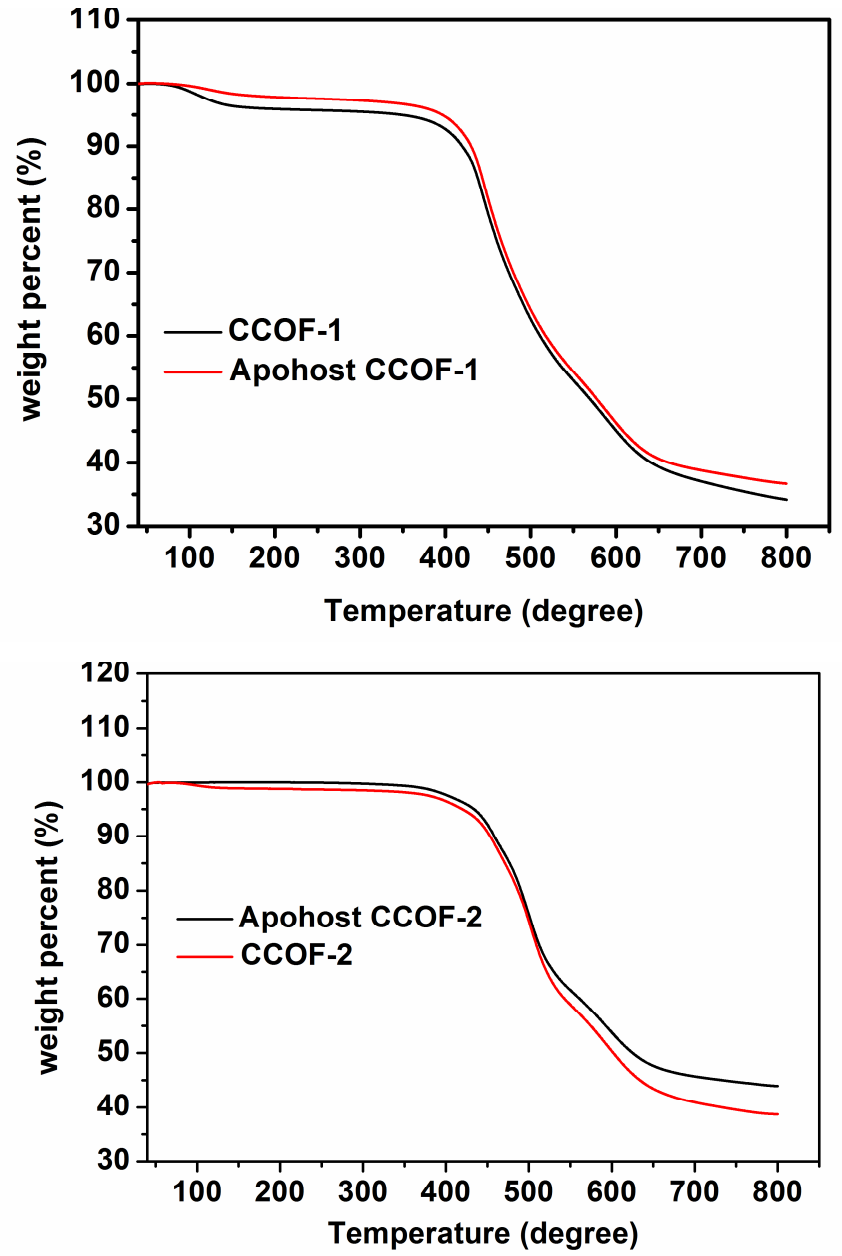
10. Figure S6. SEM images of (a) CCOF-1 and (c) CCOF-2; and TEM images of (b) CCOF-1 and (d) CCOF-2.
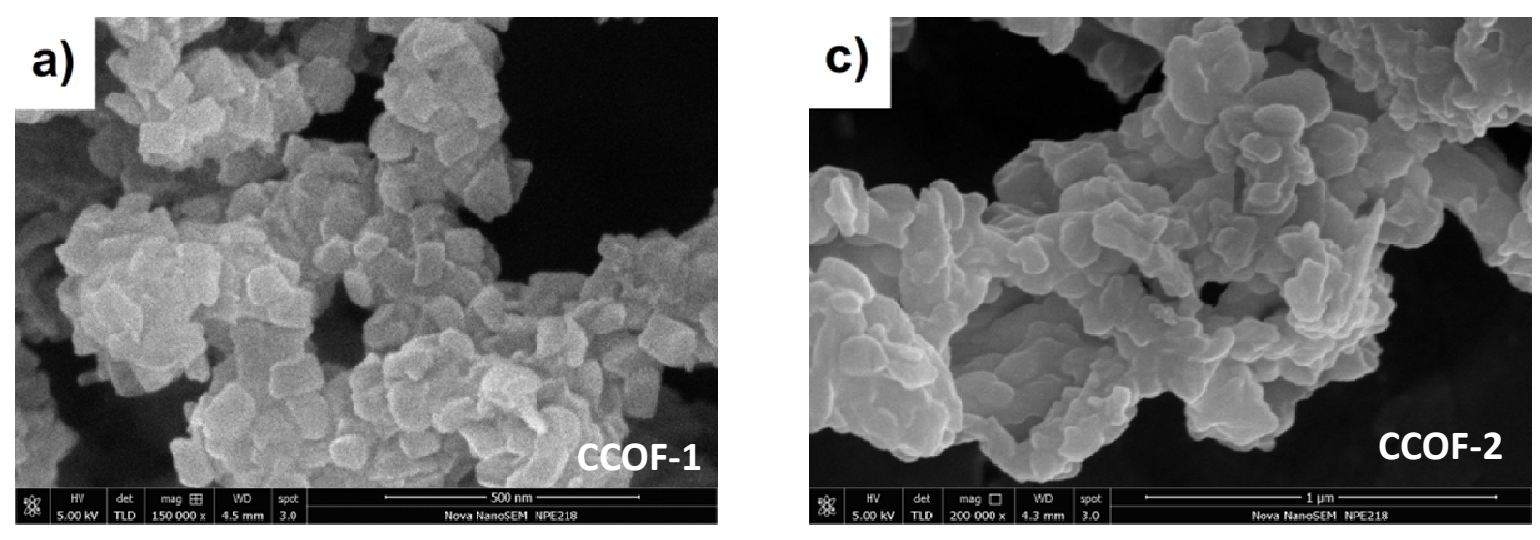

\section{b)}

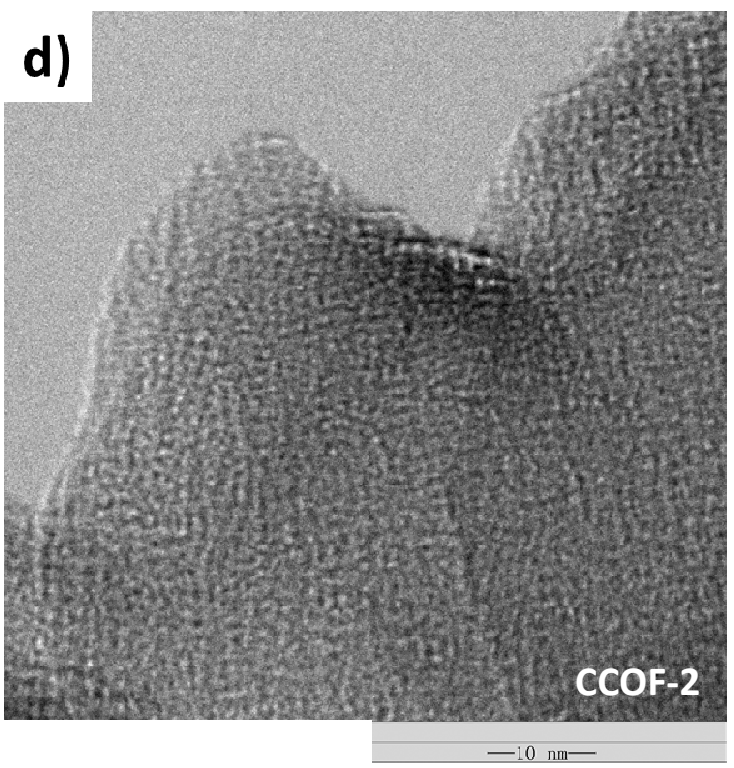




\section{Figures S7\&S8. PXRD patterns}

\subsection{Figure S7. PXRD of the CCOFs after treating by different conditions}

(a) and (c): The powders were soaked in different solvents in 10 days and dried in air.

(b) and (d): The above solvent-treated powders was solvent exchanged with THF three times and then dried at $100{ }^{\circ} \mathrm{C}$ under vacuum for $24 \mathrm{~h}$.

(e) and (f): The COFs after measuring $\mathrm{N}_{2}$ adsorption-desorption isotherms

(a)

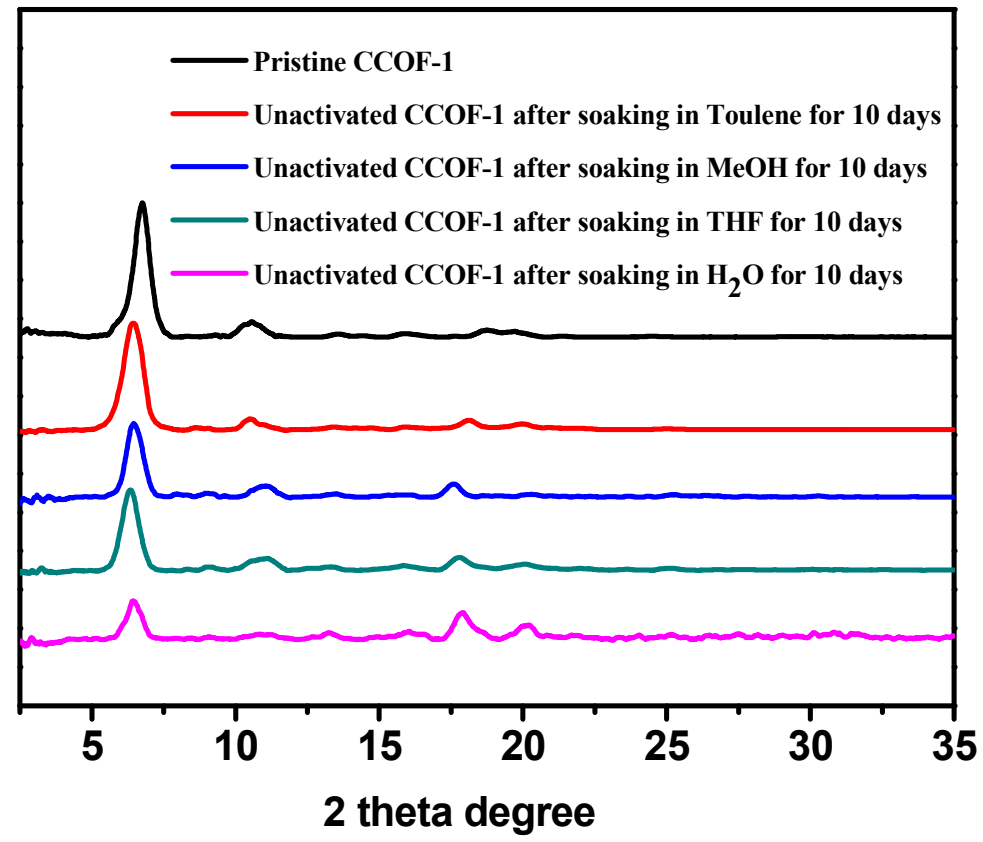

(b)

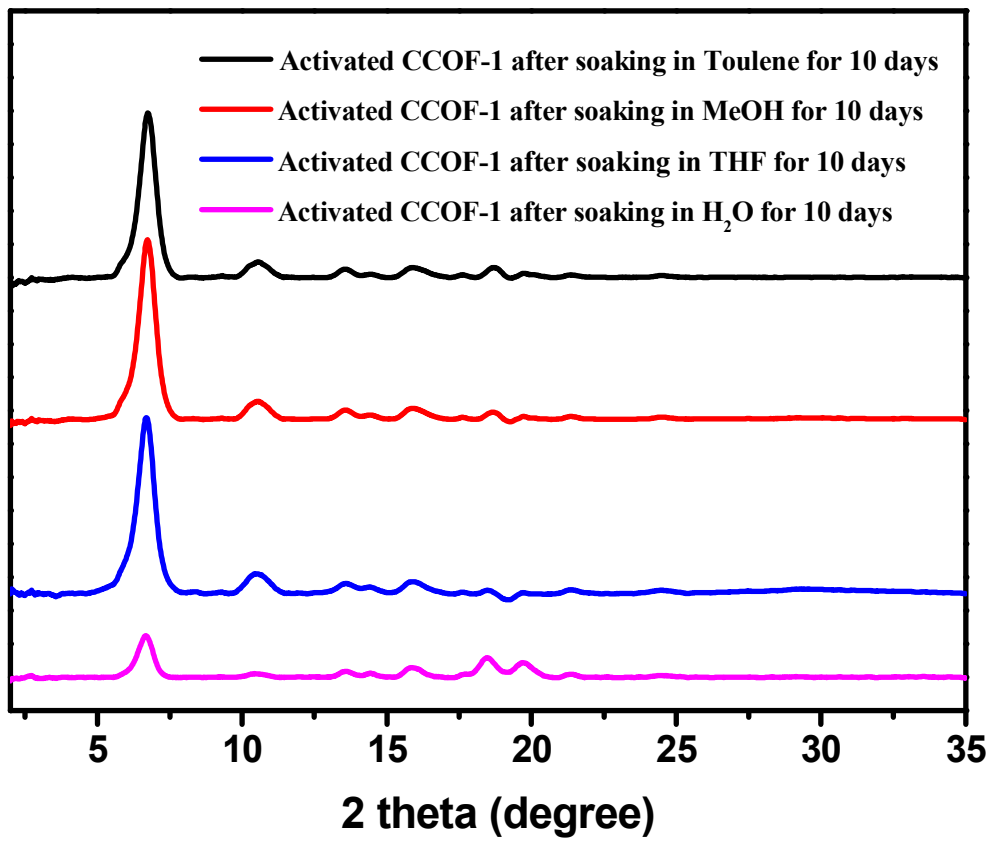


(c)

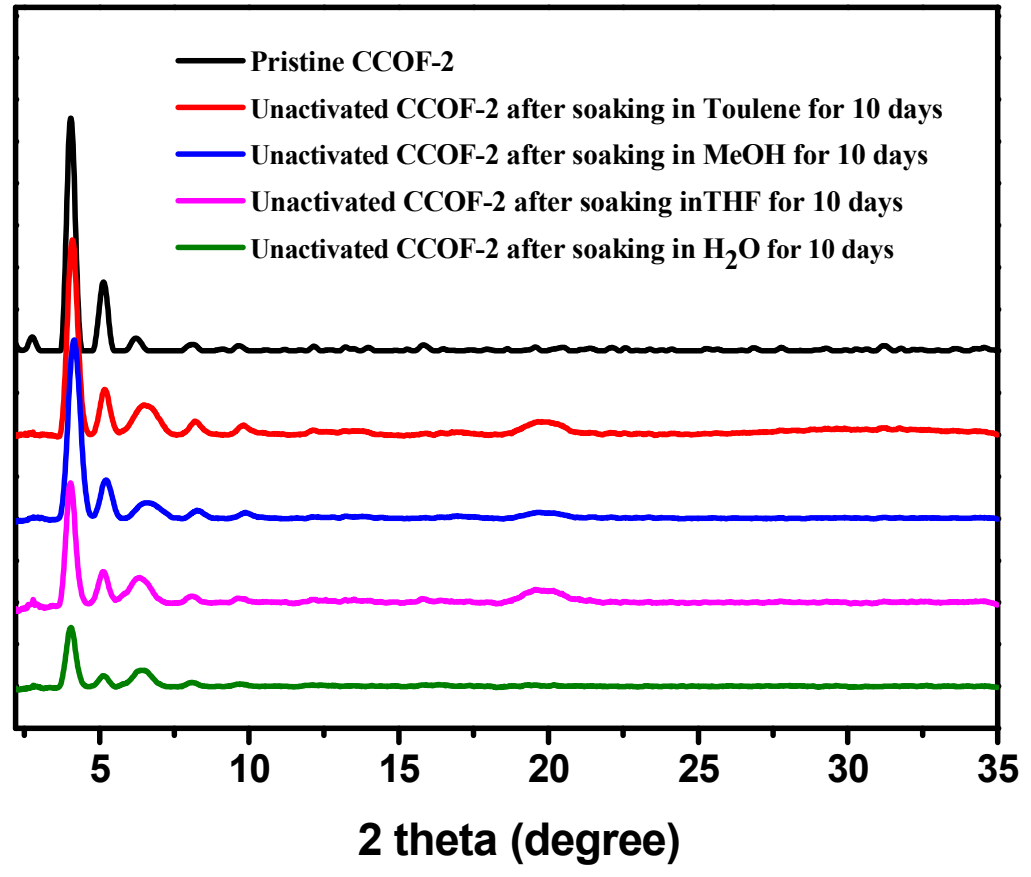

(d)

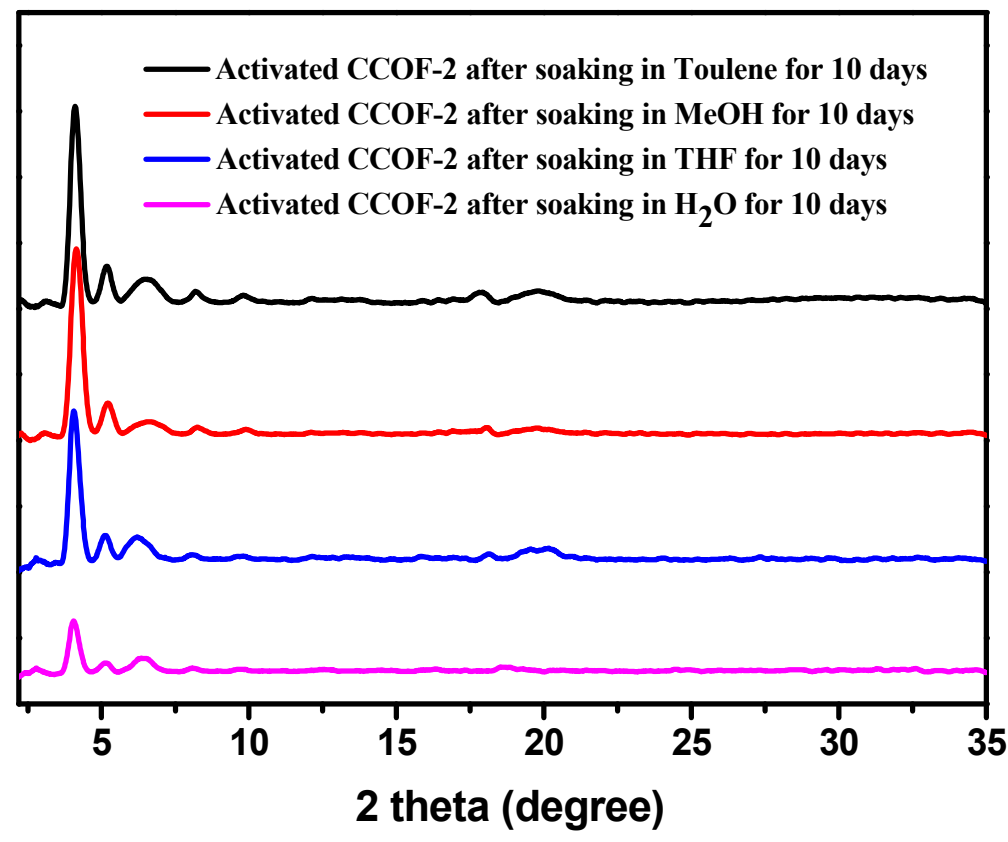


(e)

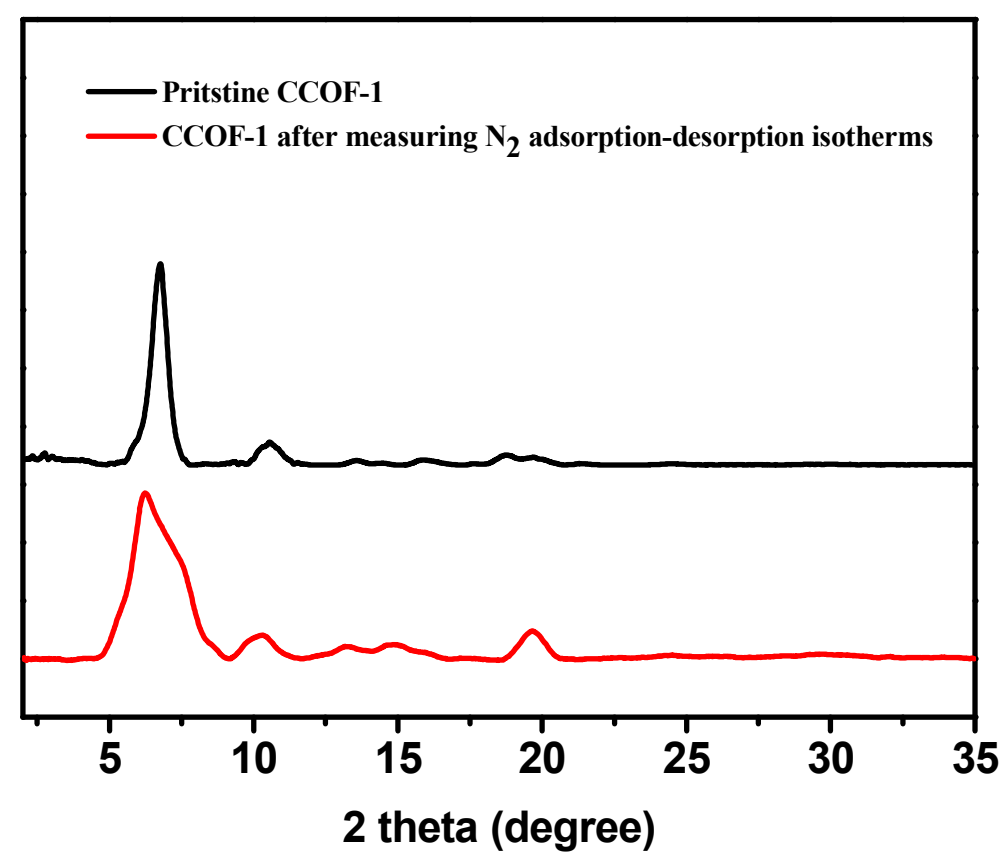

(f)

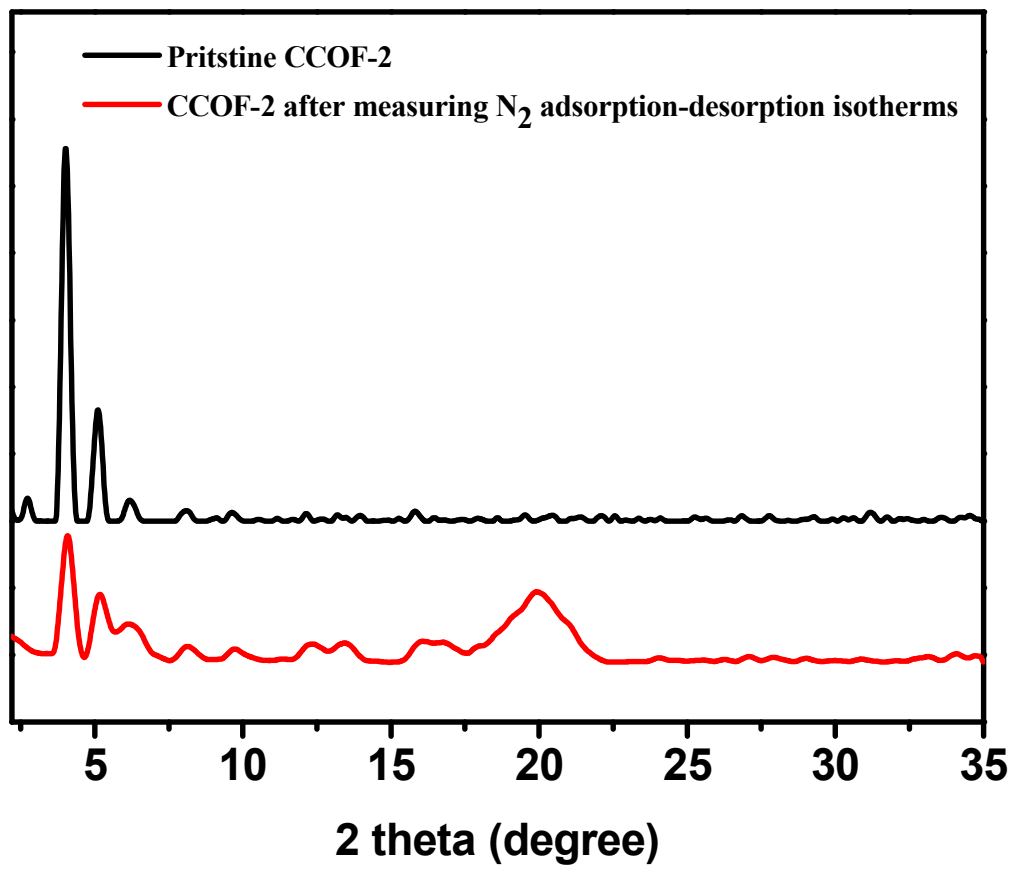


11.2. Figure S8. PXRD of the recycling catalysts

(a)

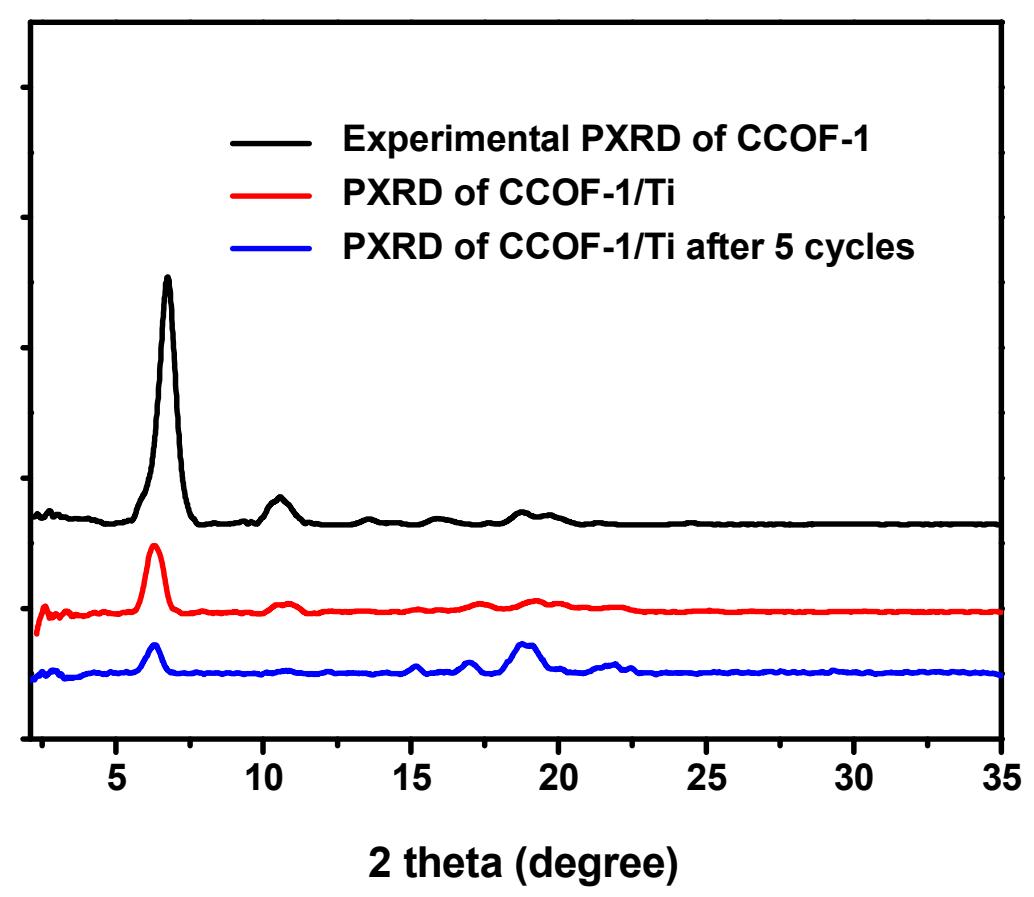

(b)

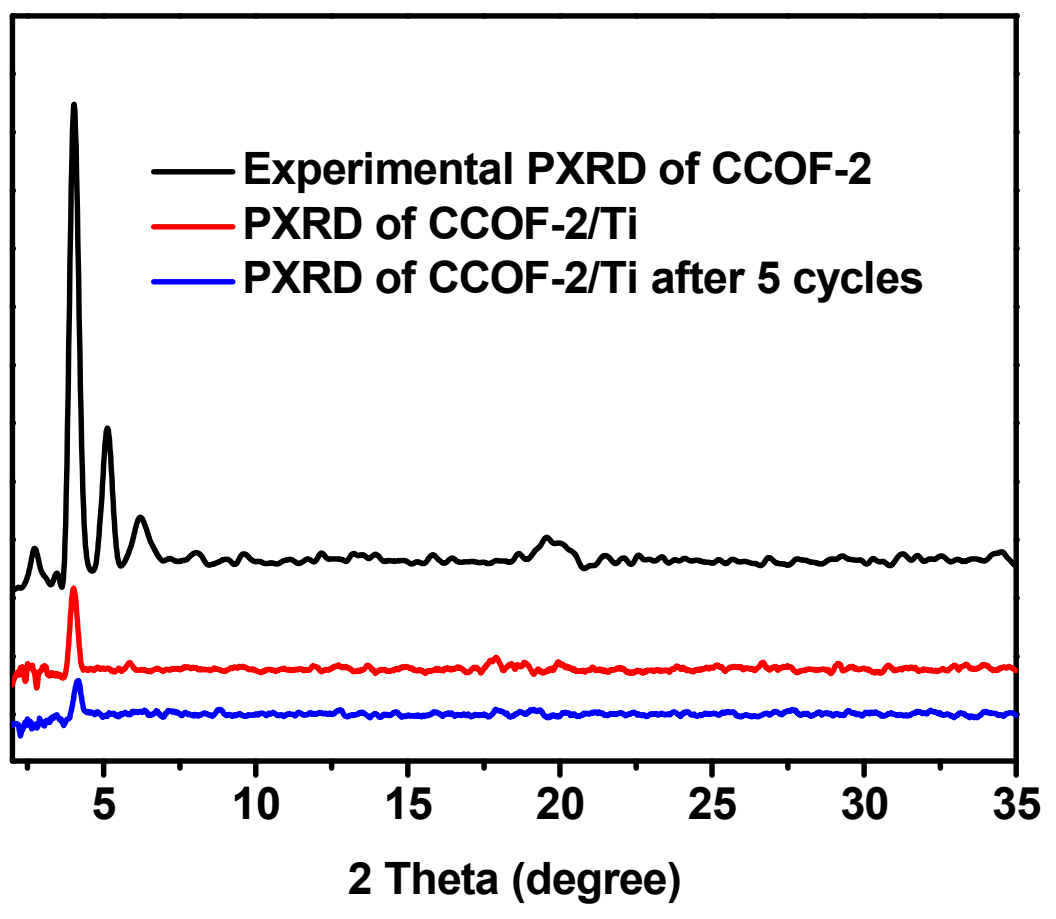


12. Figure S9. BET plots of the CCOFs
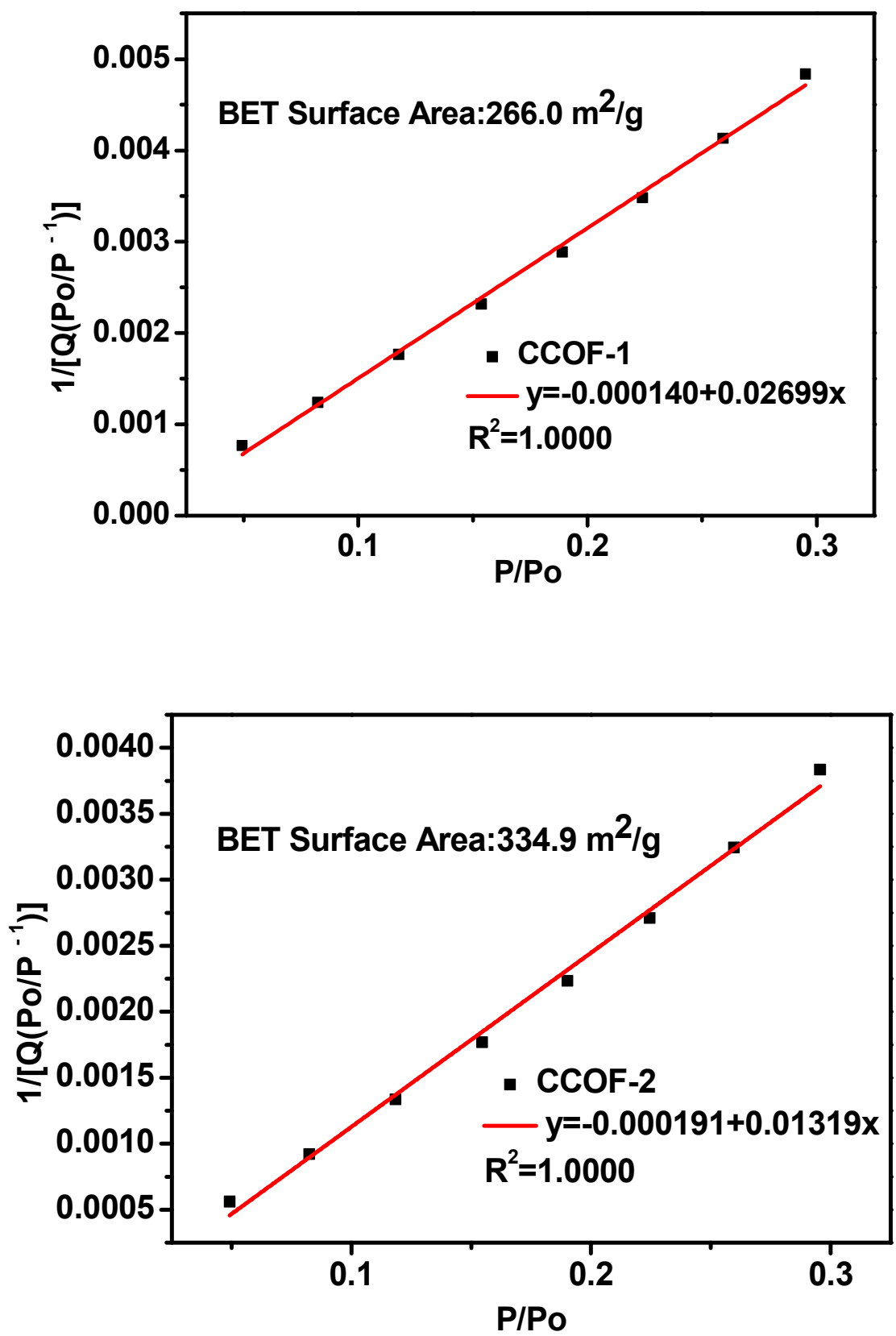


\section{Figures S10-S13. Structural modeling and PXRD Analysis of the CCOFs}

Structure Simulation: Molecular modeling of these COFs was generated with the Materials Studio (ver. 7.0) suite of programs. Pawley refinement was carried out using Reflex, a software package for crystal determination from PXRD pattern. Unit cell dimension was set to the theoretical parameters. The Pawley refinement was performed to optimize the lattice parameters iteratively until the $\mathrm{R}_{w p}$ value converges and the overlay of the observed with refined profiles shows good agreement. The lattice models (e.g., cell parameters, atomic positions, and total energies) were then fully optimized using MS Forcite molecular dynamics module (universal force fields, Ewald summations) method.

For CCOF-1, considering the geometry of the precursors and the connection patterns, only a few topologies are reasonable.

13.1. Figure S10 Space-filling models of CCOF-1 with different nets: a) sp, b) dp, c) bsp, d) tp. Carbon, gray; Nitrogen, blue; Oxygen, red; Hydrogen atoms are omitted.

a)

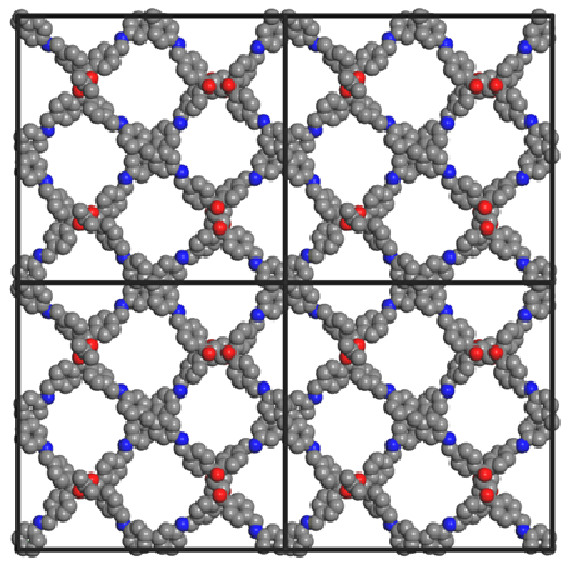

c)

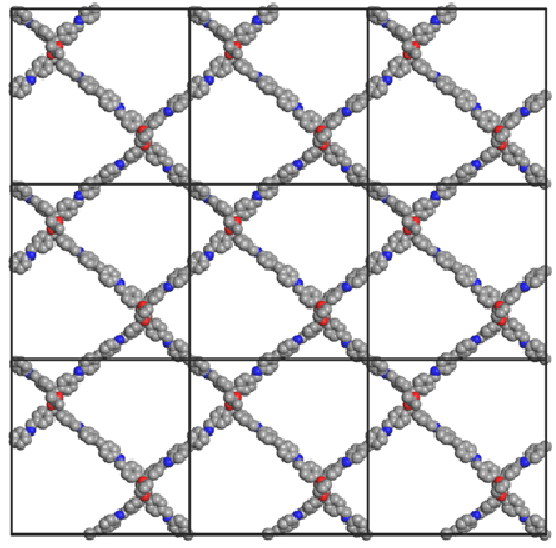

b)

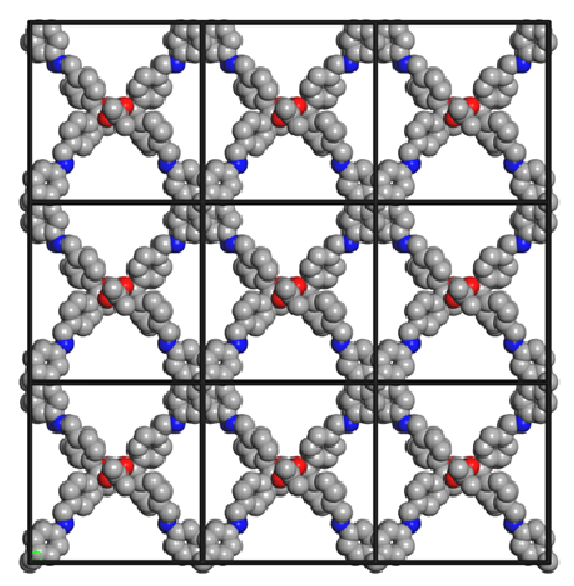

d)

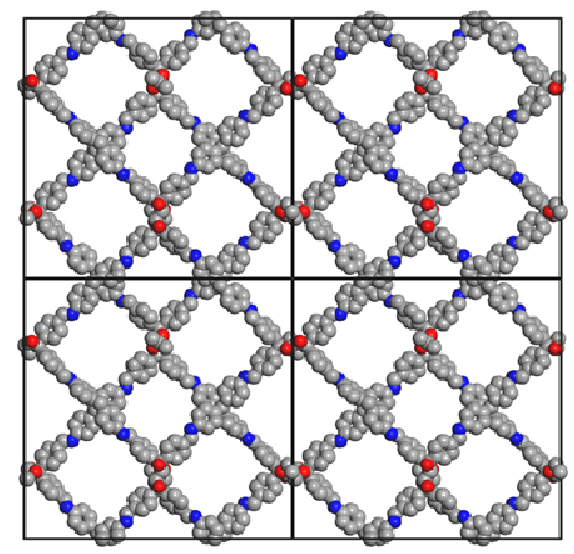


13.2. Figure S11 The calculated PXRD profiles: sp (red), dp (bule), tp (pink), bsp (green) The experimental pattern was also presented (black).

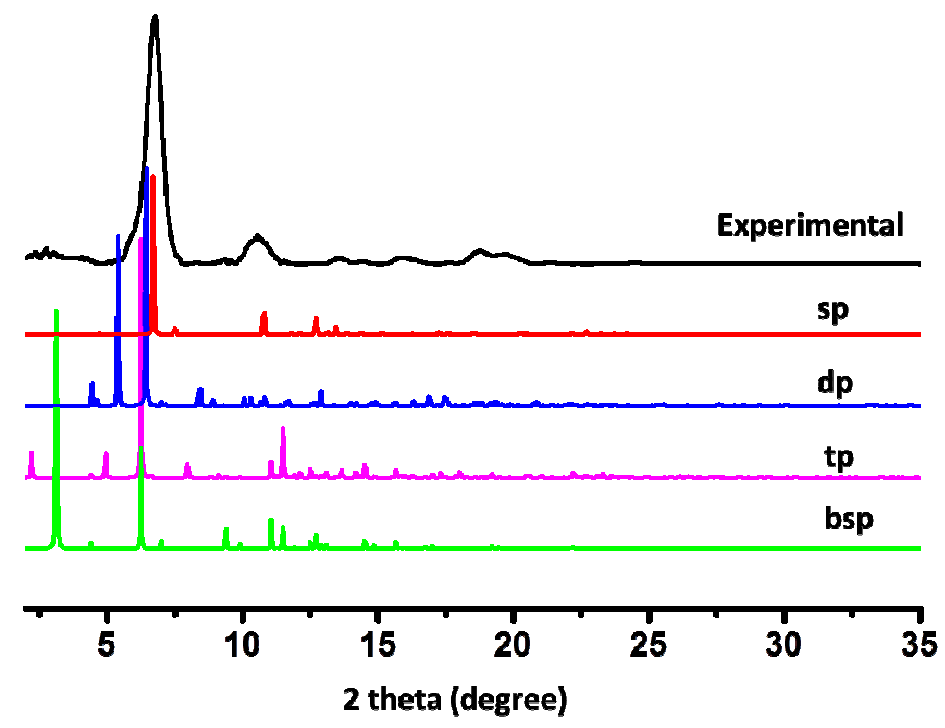

For CCOF-1 dp, tp, bsp topology, their calculated total energy $(959.8,835.4$ and $1061.5 \mathrm{kcal} / \mathrm{mol}$ respectively) is higher than sp topology (636.6 kcal / mol), and the calculated PXRD pattern (Figure S11) has shown several peaks below 6 degrees that disagree with the experimental data. For sp topology $\left(P 2_{1}\right.$ space group, also see Figure S11 in SI), the calculated PXRD pattern was in agreement with experimental data. In addition, Pawley refinement yielded an XRD pattern that is in good agreement with the experimental data and the $\mathrm{R}_{w p}$ and $\mathrm{R}_{p}$ values was $6.97 \%$ and $5.82 \%$.

For CCOF-2, considering the geometry of the precursors and the connection patterns, only a few topologies are reasonable. For sp, bdp and fp topology, their calculated total energy $(914.4,1431.1$ and $1354.5 \mathrm{kcal} / \mathrm{mol})$ is higher than tp topology $(878.8 \mathrm{kcal} / \mathrm{mol}$ ), the calculated PXRD pattern (Figure S12) has shown several peaks below 7 degrees that disagree with the experimental data. For tp topology ( $P 2_{1}$ space group, also see Figure S12 in SI), the calculated PXRD pattern was in agreement with experimental data. In addition, Pawley refinement yielded an XRD pattern that is in good agreement with the experimental data and the $\mathrm{R}_{w p}$ and $\mathrm{R}_{p}$ values was $4.25 \%$ and $3.03 \%$. 
13.3. Figure S12. Space-filling models of CCOF-2 with different nets: a) sp, b) tp, c) bdp, d) fp. Carbon, gray; Nitrogen, blue; Oxygen, red; Hydrogen atoms are omitted.

a)

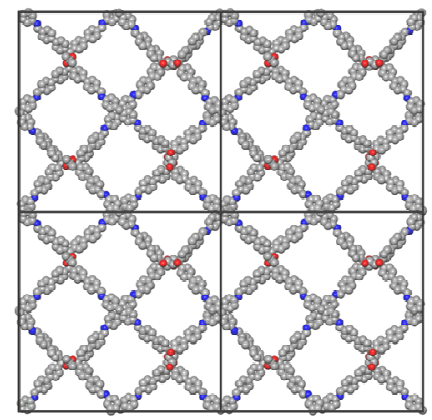

c)

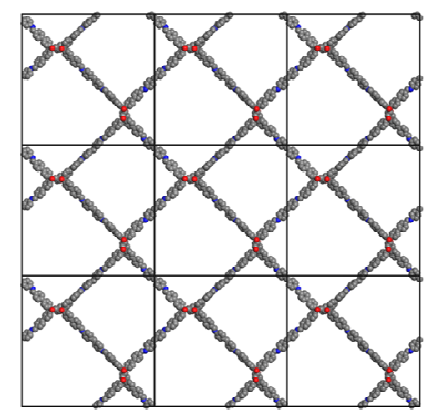

b)

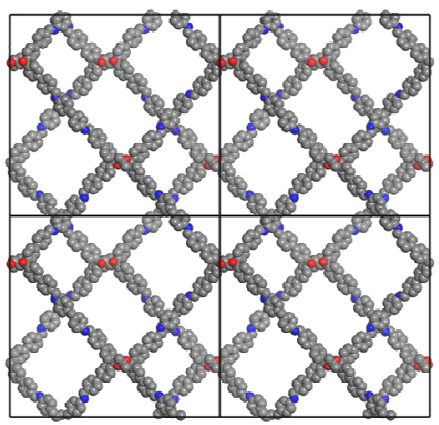

d)

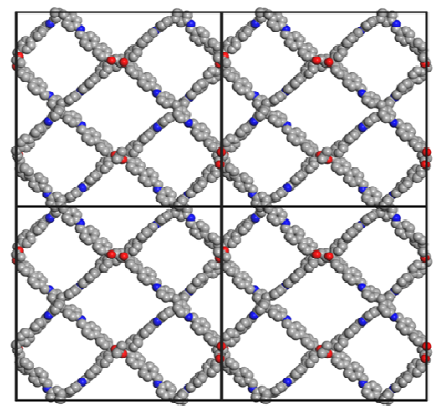

13.4. Figure S13. The calculated PXRD profiles: tp (red), sp (bule), fp (pink), bdp (green). The experimental pattern was also presented (black).

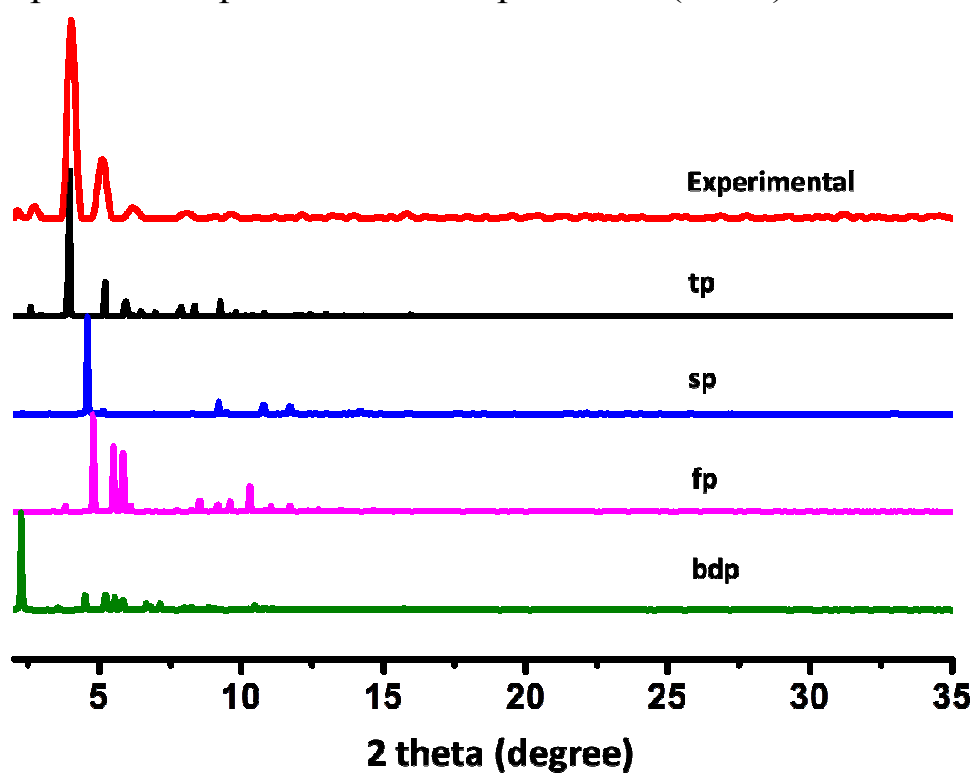

14. Table S2 and S3. Fractional atomic coordinates and unit cell parameters

14.1. Table S2. Fractional atomic coordinates of for the unit cell of $\mathbf{s p}$ net CCOF-1 with $P 2_{1}$ space group. 
CCOF-1: Space group: $P 2_{1}$

$\mathrm{a}=37.413 \AA, \mathrm{b}=37.113 \AA, \mathrm{c}=8.204 \AA$

$\alpha=90.0^{\circ}, \beta=89.113^{\circ}, \gamma=90.0^{\circ}$

\begin{tabular}{|c|c|c|c|}
\hline Atom & $\mathrm{x}$ & $\mathrm{y}$ & $\mathrm{z}$ \\
\hline $\mathrm{C} 1$ & -0.7337 & 0.75791 & 1.4875 \\
\hline $\mathrm{C} 2$ & -0.7666 & 0.73299 & 1.48745 \\
\hline $\mathrm{C} 3$ & -0.7507 & 0.74581 & 1.74623 \\
\hline $\mathrm{C} 4$ & -0.774 & 0.77015 & 1.85265 \\
\hline $\mathrm{C} 5$ & -0.7663 & 0.81875 & 1.44032 \\
\hline C6 & -0.7636 & 0.83802 & 1.58793 \\
\hline C7 & -0.7898 & 0.86261 & 1.636 \\
\hline $\mathrm{C} 8$ & -0.8196 & 0.86885 & 1.53816 \\
\hline C9 & -0.8228 & 0.84987 & 1.39139 \\
\hline $\mathrm{C} 10$ & -0.7965 & 0.82547 & 1.34239 \\
\hline C11 & -0.7354 & 0.79395 & 1.38354 \\
\hline $\mathrm{C} 12$ & -0.8467 & 0.89551 & 1.59042 \\
\hline $\mathrm{C} 13$ & -0.9047 & 0.92343 & 1.56138 \\
\hline N14 & -0.8769 & 0.89874 & 1.51491 \\
\hline $\mathrm{C} 15$ & -0.7004 & 0.81598 & 1.39236 \\
\hline $\mathrm{C} 16$ & -0.6696 & 0.80448 & 1.47656 \\
\hline $\mathrm{C} 17$ & -0.639 & 0.82579 & 1.48112 \\
\hline $\mathrm{C} 18$ & -0.6377 & 0.85915 & 1.40179 \\
\hline $\mathrm{C} 19$ & -0.6677 & 0.87065 & 1.31523 \\
\hline $\mathrm{C} 20$ & -0.6984 & 0.84927 & 1.30961 \\
\hline $\mathrm{C} 21$ & -0.7276 & 0.72156 & 1.85294 \\
\hline $\mathrm{C} 22$ & -0.6045 & 0.88085 & 1.40779 \\
\hline N23 & -0.603 & 0.91287 & 1.34389 \\
\hline $\mathrm{C} 24$ & -0.5723 & 0.9361 & 1.33313 \\
\hline $\mathrm{C} 25$ & -0.7336 & 0.67204 & 1.44706 \\
\hline $\mathrm{C} 26$ & -0.7028 & 0.66521 & 1.35171 \\
\hline $\mathrm{C} 27$ & -0.6769 & 0.6408 & 1.4031 \\
\hline $\mathrm{C} 28$ & -0.681 & 0.6219 & 1.54973 \\
\hline $\mathrm{C} 29$ & -0.7113 & 0.62839 & 1.64549 \\
\hline C30 & -0.7372 & 0.65302 & 1.59508 \\
\hline C31 & -0.7643 & 0.69655 & 1.38661 \\
\hline C32 & -0.6545 & 0.59488 & 1.60339 \\
\hline C33 & -0.5973 & 0.56535 & 1.57536 \\
\hline N34 & -0.6242 & 0.59088 & 1.52828 \\
\hline $\mathrm{C} 35$ & -0.7993 & 0.67446 & 1.39503 \\
\hline C36 & -0.8006 & 0.64074 & 1.31581 \\
\hline C37 & -0.8312 & 0.61926 & 1.32146 \\
\hline C38 & -0.8619 & 0.63111 & 1.40398 \\
\hline
\end{tabular}




\begin{tabular}{|c|c|c|c|}
\hline C39 & -0.8612 & 0.66491 & 1.47949 \\
\hline $\mathrm{C} 40$ & -0.8306 & 0.68631 & 1.47533 \\
\hline $\mathrm{C} 41$ & -0.8949 & 0.60926 & 1.40928 \\
\hline N42 & -0.8954 & 0.57636 & 1.35518 \\
\hline $\mathrm{C} 43$ & -0.9257 & 0.55271 & 1.34525 \\
\hline $\mathrm{O} 44$ & -0.728 & 0.76715 & 1.65012 \\
\hline $\mathrm{O} 45$ & -0.7599 & 0.70475 & 1.22065 \\
\hline O46 & -0.7732 & 0.7244 & 1.6504 \\
\hline $\mathrm{O} 47$ & -0.739 & 0.7851 & 1.21802 \\
\hline $\mathrm{C} 48$ & -0.8973 & 0.95665 & 1.63631 \\
\hline C49 & -0.925 & 0.97796 & 1.69755 \\
\hline $\mathrm{C} 50$ & -0.9605 & 0.96657 & 1.68471 \\
\hline $\mathrm{C} 51$ & -0.9679 & 0.93457 & 1.60056 \\
\hline $\mathrm{C} 52$ & -0.9402 & 0.91348 & 1.53725 \\
\hline C53 & -0.6061 & 0.53213 & 1.64724 \\
\hline $\mathrm{C} 54$ & -0.5794 & 0.50976 & 1.70843 \\
\hline $\mathrm{C} 55$ & -0.5435 & 0.52006 & 1.69867 \\
\hline $\mathrm{C} 56$ & -0.5347 & 0.55218 & 1.61827 \\
\hline $\mathrm{C} 57$ & -0.5614 & 0.57432 & 1.55454 \\
\hline $\mathrm{C} 58$ & -0.9611 & 0.5651 & 1.35229 \\
\hline C59 & -0.9895 & 0.54149 & 1.32157 \\
\hline $\mathrm{C} 60$ & -0.983 & 0.5052 & 1.28416 \\
\hline C61 & -0.9195 & 0.51627 & 1.31266 \\
\hline C62 & -0.5373 & 0.92401 & 1.35979 \\
\hline C63 & -0.5082 & 0.94672 & 1.3279 \\
\hline C64 & -0.5136 & 0.98191 & 1.27085 \\
\hline C65 & -0.5486 & 0.99427 & 1.25036 \\
\hline C66 & -0.5775 & 0.97155 & 1.28058 \\
\hline C67 & -0.4833 & 1.00226 & 1.19172 \\
\hline C68 & -0.9884 & 0.98271 & 1.79472 \\
\hline C69 & 0.23603 & 0.21941 & 0.5148 \\
\hline $\mathrm{C} 70$ & 0.2652 & 0.24842 & 0.5001 \\
\hline C71 & 0.25352 & 0.23667 & 0.76513 \\
\hline $\mathrm{C} 72$ & 0.27513 & 0.21008 & 0.86666 \\
\hline $\mathrm{C} 73$ & 0.17482 & 0.19265 & 0.42983 \\
\hline $\mathrm{C} 74$ & 0.18205 & 0.16154 & 0.52355 \\
\hline $\mathrm{C} 75$ & 0.15793 & 0.13296 & 0.53058 \\
\hline C76 & 0.1259 & 0.13421 & 0.44454 \\
\hline C77 & 0.11829 & 0.16486 & 0.351 \\
\hline C78 & 0.14244 & 0.19354 & 0.34349 \\
\hline C79 & 0.20033 & 0.22527 & 0.41613 \\
\hline $\mathrm{C} 80$ & 0.10199 & 0.10253 & 0.44888 \\
\hline $\mathrm{C} 81$ & 0.04868 & 0.07162 & 0.35406 \\
\hline
\end{tabular}




\begin{tabular}{|c|c|c|c|}
\hline N82 & 0.07188 & 0.10226 & 0.37139 \\
\hline $\mathrm{C} 83$ & 0.17989 & 0.25901 & 0.47644 \\
\hline $\mathrm{C} 84$ & 0.16183 & 0.25808 & 0.62753 \\
\hline $\mathrm{C} 85$ & 0.14025 & 0.28662 & 0.67797 \\
\hline $\mathrm{C} 86$ & 0.13599 & 0.31712 & 0.57959 \\
\hline $\mathrm{C} 87$ & 0.15433 & 0.31889 & 0.43068 \\
\hline $\mathrm{C} 88$ & 0.17575 & 0.29019 & 0.37911 \\
\hline C89 & 0.23493 & 0.26407 & 0.87561 \\
\hline $\mathrm{C} 90$ & 0.11085 & 0.34557 & 0.6316 \\
\hline N91 & 0.10836 & 0.37623 & 0.55559 \\
\hline $\mathrm{C} 92$ & 0.08281 & 0.40341 & 0.59999 \\
\hline C93 & 0.32519 & 0.2701 & 0.37042 \\
\hline C94 & 0.35482 & 0.26479 & 0.26708 \\
\hline C95 & 0.38133 & 0.29084 & 0.25153 \\
\hline C96 & 0.37914 & 0.32324 & 0.33808 \\
\hline C97 & 0.34932 & 0.32949 & 0.43789 \\
\hline C98 & 0.32256 & 0.3034 & 0.45334 \\
\hline C99 & 0.29877 & 0.23859 & 0.3911 \\
\hline $\mathrm{C} 100$ & 0.40803 & 0.34999 & 0.31889 \\
\hline C101 & 0.43393 & 0.40846 & 0.37574 \\
\hline N102 & 0.4076 & 0.38063 & 0.39565 \\
\hline $\mathrm{C} 103$ & 0.32022 & 0.2067 & 0.46293 \\
\hline $\mathrm{C} 104$ & 0.32285 & 0.17295 & 0.38371 \\
\hline $\mathrm{C} 105$ & 0.34615 & 0.14636 & 0.43984 \\
\hline C106 & 0.36775 & 0.15274 & 0.57552 \\
\hline C107 & 0.36479 & 0.18571 & 0.65724 \\
\hline C108 & 0.34154 & 0.21227 & 0.60175 \\
\hline C109 & 0.3948 & 0.12649 & 0.62946 \\
\hline N110 & 0.3984 & 0.09513 & 0.56048 \\
\hline C111 & 0.42571 & 0.06977 & 0.60356 \\
\hline $\mathrm{O} 112$ & 0.22767 & 0.21713 & 0.68006 \\
\hline O113 & 0.2873 & 0.23067 & 0.23229 \\
\hline $\mathrm{O} 114$ & 0.27636 & 0.25543 & 0.65891 \\
\hline O115 & 0.20832 & 0.22814 & 0.24892 \\
\hline C116 & 0.01366 & 0.07729 & 0.30221 \\
\hline C117 & -0.0083 & 0.04817 & 0.26231 \\
\hline C118 & 0.00444 & 0.01273 & 0.27299 \\
\hline C119 & 0.03916 & 0.00702 & 0.32913 \\
\hline $\mathrm{C} 120$ & 0.06105 & 0.03601 & 0.36987 \\
\hline C121 & 0.46885 & 0.40117 & 0.32204 \\
\hline C122 & 0.49179 & 0.42938 & 0.28009 \\
\hline C123 & 0.48051 & 0.46529 & 0.29325 \\
\hline $\mathrm{C} 124$ & 0.4466 & 0.47244 & 0.35769 \\
\hline
\end{tabular}




\begin{tabular}{|c|c|c|c|}
\hline $\mathrm{C} 125$ & 0.42374 & 0.44428 & 0.40072 \\
\hline C126 & 0.45939 & 0.08128 & 0.65571 \\
\hline $\mathrm{C} 127$ & 0.48457 & 0.05635 & 0.70823 \\
\hline C128 & 0.47694 & 0.01934 & 0.70759 \\
\hline C129 & 0.44393 & 0.00768 & 0.64869 \\
\hline C130 & 0.41865 & 0.03276 & 0.59521 \\
\hline C131 & 0.04852 & 0.39405 & 0.6539 \\
\hline C132 & 0.02523 & 0.4204 & 0.71146 \\
\hline C133 & 0.03552 & 0.45674 & 0.71536 \\
\hline C134 & 0.06907 & 0.46638 & 0.65361 \\
\hline C135 & 0.09234 & 0.4399 & 0.59384 \\
\hline C136 & 0.01422 & 0.48285 & 0.81671 \\
\hline C137 & 0.4995 & 0.49418 & 0.19823 \\
\hline C138 & 0.94774 & 0.99273 & 1.71696 \\
\hline H139 & -0.7105 & 0.74194 & 1.44492 \\
\hline $\mathrm{H} 140$ & -0.7895 & 0.74878 & 1.44197 \\
\hline H141 & -0.7887 & 0.75399 & 1.94475 \\
\hline H142 & -0.7939 & 0.78424 & 1.77625 \\
\hline H143 & -0.7575 & 0.7907 & 1.91476 \\
\hline H144 & -0.7406 & 0.83468 & 1.66517 \\
\hline H145 & -0.7866 & 0.87701 & 1.74974 \\
\hline H146 & -0.8454 & 0.85434 & 1.31246 \\
\hline H147 & -0.8002 & 0.81267 & 1.22569 \\
\hline H148 & -0.8415 & 0.91126 & 1.69887 \\
\hline H149 & -0.6684 & 0.77901 & 1.53926 \\
\hline H150 & -0.616 & 0.81606 & 1.54711 \\
\hline H151 & -0.6675 & 0.89607 & 1.25082 \\
\hline H152 & -0.721 & 0.85892 & 1.24085 \\
\hline H153 & -0.7075 & 0.70751 & 1.77683 \\
\hline H154 & -0.7442 & 0.70099 & 1.91498 \\
\hline H155 & -0.7131 & 0.73779 & 1.94495 \\
\hline H156 & -0.5816 & 0.86935 & 1.46701 \\
\hline H157 & -0.6984 & 0.67786 & 1.23504 \\
\hline H158 & -0.6539 & 0.6362 & 1.32591 \\
\hline H159 & -0.7152 & 0.61407 & 1.75915 \\
\hline H160 & -0.7606 & 0.65652 & 1.67059 \\
\hline H161 & -0.6604 & 0.57928 & 1.71177 \\
\hline H162 & -0.7776 & 0.63081 & 1.24994 \\
\hline H163 & -0.8309 & 0.59349 & 1.25995 \\
\hline H164 & -0.8846 & 0.6749 & 1.54234 \\
\hline H165 & -0.8322 & 0.71209 & 1.53521 \\
\hline H166 & -0.9187 & 0.62123 & 1.46074 \\
\hline H167 & -0.7375 & 0.71843 & 1.20744 \\
\hline
\end{tabular}




\begin{tabular}{|c|c|c|c|}
\hline H168 & -0.7614 & 0.77146 & 1.2047 \\
\hline H169 & -0.8702 & 0.96565 & 1.65193 \\
\hline $\mathrm{H} 170$ & -0.9185 & 1.00236 & 1.76323 \\
\hline H171 & -0.9949 & 0.92471 & 1.59438 \\
\hline H172 & -0.9464 & 0.88814 & 1.47878 \\
\hline H173 & -0.6335 & 0.52388 & 1.66017 \\
\hline H174 & -0.587 & 0.4853 & 1.77132 \\
\hline $\mathrm{H} 175$ & -0.5073 & 0.56128 & 1.61529 \\
\hline H176 & -0.5541 & 0.59966 & 1.49839 \\
\hline H177 & -0.9671 & 0.59317 & 1.37297 \\
\hline $\mathrm{H} 178$ & -1.0163 & 0.55201 & 1.31727 \\
\hline H179 & -0.8925 & 0.50628 & 1.30489 \\
\hline H180 & -0.5319 & 0.89667 & 1.39669 \\
\hline H181 & -0.4815 & 0.93611 & 1.3381 \\
\hline H182 & -0.5535 & 1.02094 & 1.20234 \\
\hline H183 & -0.6042 & 0.98128 & 1.25781 \\
\hline H184 & -0.4943 & 1.02401 & 1.11426 \\
\hline H185 & -0.4693 & 0.98318 & 1.10899 \\
\hline H186 & -0.9755 & 0.99948 & 1.88965 \\
\hline H187 & -1.0011 & 0.95977 & 1.85915 \\
\hline H188 & 0.24778 & 0.19323 & 0.47776 \\
\hline H189 & 0.25305 & 0.27349 & 0.45194 \\
\hline H190 & 0.28551 & 0.18817 & 0.7878 \\
\hline H191 & 0.25798 & 0.19743 & 0.9611 \\
\hline H192 & 0.29798 & 0.22387 & 0.92472 \\
\hline H193 & 0.20623 & 0.15837 & 0.592 \\
\hline H194 & 0.16457 & 0.10952 & 0.60282 \\
\hline H195 & 0.09387 & 0.16656 & 0.28208 \\
\hline H196 & 0.13585 & 0.21669 & 0.26939 \\
\hline H197 & 0.11061 & 0.07908 & 0.51607 \\
\hline H198 & 0.16332 & 0.23456 & 0.70491 \\
\hline H199 & 0.12623 & 0.2845 & 0.79321 \\
\hline $\mathrm{H} 200$ & 0.1513 & 0.34199 & 0.35155 \\
\hline H201 & 0.1881 & 0.29266 & 0.26063 \\
\hline H202 & 0.2134 & 0.25085 & 0.9464 \\
\hline $\mathrm{H} 203$ & 0.22281 & 0.28567 & 0.80071 \\
\hline $\mathrm{H} 204$ & 0.2542 & 0.27667 & 0.96042 \\
\hline $\mathrm{H} 205$ & 0.09438 & 0.3404 & 0.73787 \\
\hline H206 & 0.35777 & 0.23984 & 0.20008 \\
\hline H207 & 0.40384 & 0.28547 & 0.17235 \\
\hline $\mathrm{H} 208$ & 0.34667 & 0.35452 & 0.50445 \\
\hline H209 & 0.30034 & 0.30972 & 0.53183 \\
\hline $\mathrm{H} 210$ & 0.42892 & 0.34413 & 0.23163 \\
\hline
\end{tabular}




\begin{tabular}{|c|c|c|c|}
$\mathrm{H} 211$ & 0.30806 & 0.16686 & 0.27483 \\
\hline $\mathrm{H} 212$ & 0.34799 & 0.12119 & 0.37384 \\
\hline $\mathrm{H} 213$ & 0.38126 & 0.19138 & 0.76204 \\
\hline $\mathrm{H} 214$ & 0.34129 & 0.23788 & 0.66451 \\
\hline $\mathrm{H} 215$ & 0.41192 & 0.13404 & 0.72965 \\
\hline $\mathrm{H} 216$ & 0.27117 & 0.2097 & 0.23622 \\
\hline $\mathrm{H} 217$ & 0.22457 & 0.24874 & 0.2324 \\
\hline $\mathrm{H} 218$ & 0.00381 & 0.10444 & 0.28688 \\
\hline $\mathrm{H} 219$ & -0.0346 & 0.05341 & 0.21434 \\
\hline $\mathrm{H} 220$ & 0.05025 & -0.0199 & 0.32998 \\
\hline $\mathrm{H} 221$ & 0.08823 & 0.0303 & 0.40316 \\
\hline $\mathrm{H} 222$ & 0.47816 & 0.37383 & 0.30727 \\
\hline $\mathrm{H} 223$ & 0.51764 & 0.42313 & 0.22862 \\
\hline $\mathrm{H} 224$ & 0.43684 & 0.49982 & 0.36302 \\
\hline $\mathrm{H} 225$ & 0.3971 & 0.45043 & 0.44283 \\
\hline $\mathrm{H} 226$ & 0.46613 & 0.10958 & 0.65824 \\
\hline $\mathrm{H} 227$ & 0.50965 & 0.06615 & 0.75489 \\
\hline $\mathrm{H} 228$ & 0.43715 & -0.0206 & 0.65115 \\
\hline $\mathrm{H} 229$ & 0.39296 & 0.02342 & 0.55392 \\
\hline $\mathrm{H} 230$ & 0.04002 & 0.36622 & 0.65515 \\
\hline $\mathrm{H} 231$ & -0.0004 & 0.41212 & 0.75966 \\
\hline $\mathrm{H} 232$ & 0.07784 & 0.49411 & 0.6591 \\
\hline $\mathrm{H} 233$ & 0.11852 & 0.44763 & 0.55101 \\
\hline $\mathrm{H} 234$ & 0.03187 & 0.49422 & 0.912 \\
\hline $\mathrm{H} 235$ & -0.0067 & 0.46754 & 0.10035 \\
\hline $\mathrm{H} 236$ & 0.47856 & 0.51019 & 1.74939 \\
\hline $\mathrm{H} 237$ & 0.51591 & 0.48174 & \\
\hline $\mathrm{H} 238$ & 0.94185 & 0.96511 & \\
\hline & & & \\
\hline & & & \\
\hline
\end{tabular}

14.2 Table S3. Fractional atomic coordinates for the unit cell of tp net CCOF-2 with $P 2_{1}$ space group.

\begin{tabular}{|c|c|c|c|}
\hline \multicolumn{4}{|c|}{ CCOF-2: Space group: $\boldsymbol{P} 2_{1}$} \\
\hline \multicolumn{4}{|c|}{$\mathrm{a}=48.144 \AA, \mathrm{b}=48.075 \AA, \mathrm{c}=29.846 \AA$} \\
\hline \multicolumn{4}{|c|}{$\alpha=90.0^{\circ}, \beta=90.241^{\circ}, \gamma=90.0^{\circ}$} \\
\hline Atom & $\mathrm{x}$ & $\mathrm{y}$ & $\mathrm{z}$ \\
\hline $\mathrm{C} 1$ & 2.11619 & 1.10093 & 0.32788 \\
\hline $\mathrm{C} 2$ & 1.22513 & 1.52126 & 0.10392 \\
\hline $\mathrm{C} 3$ & 1.31192 & 1.84672 & 0.32253 \\
\hline $\mathrm{C} 4$ & 1.29122 & 1.86653 & 0.3294 \\
\hline $\mathrm{C} 5$ & 1.29805 & 1.8945 & 0.33715 \\
\hline C6 & 1.32614 & 1.90276 & 0.33689 \\
\hline $\mathrm{C} 7$ & 1.34753 & 1.8834 & 0.33027 \\
\hline
\end{tabular}




\begin{tabular}{|c|c|c|c|}
\hline $\mathrm{C} 8$ & 1.37822 & 1.64362 & 0.24953 \\
\hline C9 & 1.36218 & 1.6207 & 0.23648 \\
\hline $\mathrm{C} 10$ & 1.3343 & 1.6241 & 0.22371 \\
\hline $\mathrm{C} 11$ & 1.32265 & 1.65092 & 0.22451 \\
\hline $\mathrm{C} 12$ & 1.33869 & 1.67385 & 0.23745 \\
\hline $\mathrm{C} 13$ & 1.5935 & 1.85672 & 0.2388 \\
\hline $\mathrm{C} 14$ & 1.60965 & 1.87949 & 0.22567 \\
\hline $\mathrm{C} 15$ & 1.59811 & 1.90634 & 0.22404 \\
\hline $\mathrm{C} 16$ & 1.5702 & 1.90993 & 0.23617 \\
\hline $\mathrm{C} 17$ & 1.55405 & 1.88718 & 0.24942 \\
\hline $\mathrm{C} 18$ & 1.58293 & 1.64747 & 0.33237 \\
\hline $\mathrm{C} 19$ & 1.60401 & 1.62769 & 0.3385 \\
\hline $\mathrm{C} 20$ & 1.6321 & 1.63572 & 0.33771 \\
\hline $\mathrm{C} 21$ & 1.63865 & 1.66371 & 0.32962 \\
\hline $\mathrm{C} 22$ & 1.61764 & 1.68344 & 0.32356 \\
\hline $\mathrm{C} 23$ & 1.27542 & 1.91438 & 0.34746 \\
\hline $\mathrm{C} 24$ & 1.65487 & 1.61586 & 0.34735 \\
\hline $\mathrm{C} 25$ & 1.81466 & 1.42948 & 0.32714 \\
\hline $\mathrm{C} 26$ & 1.95326 & 1.25528 & 0.2448 \\
\hline $\mathrm{C} 27$ & 1.97785 & 1.2758 & 0.24499 \\
\hline $\mathrm{C} 28$ & 1.96536 & 1.26577 & 0.31612 \\
\hline $\mathrm{C} 29$ & 1.9794 & 1.24412 & 0.3463 \\
\hline $\mathrm{C} 30$ & 1.90488 & 1.24042 & 0.21282 \\
\hline C31 & 1.90674 & 1.21475 & 0.23581 \\
\hline $\mathrm{C} 32$ & 1.88567 & 1.19477 & 0.23193 \\
\hline C33 & 1.86215 & 1.19972 & 0.20509 \\
\hline C34 & 1.8603 & 1.22504 & 0.18186 \\
\hline $\mathrm{C} 35$ & 1.88129 & 1.24497 & 0.1856 \\
\hline $\mathrm{C} 36$ & 1.92692 & 1.26373 & 0.21563 \\
\hline C37 & 1.75857 & 1.07188 & 0.16244 \\
\hline N38 & 1.77867 & 1.09263 & 0.17541 \\
\hline C39 & 1.91239 & 1.2899 & 0.23488 \\
\hline $\mathrm{C} 40$ & 1.89609 & 1.28775 & 0.27399 \\
\hline $\mathrm{C} 41$ & 1.88074 & 1.31038 & 0.28989 \\
\hline $\mathrm{C} 42$ & 1.8811 & 1.33596 & 0.26723 \\
\hline $\mathrm{C} 43$ & 1.89754 & 1.33847 & 0.22872 \\
\hline $\mathrm{C} 44$ & 1.9128 & 1.31585 & 0.21264 \\
\hline $\mathrm{C} 45$ & 1.95103 & 1.28779 & 0.34504 \\
\hline N46 & 1.78958 & 1.4263 & 0.34241 \\
\hline $\mathrm{C} 47$ & 1.77184 & 1.44762 & 0.3606 \\
\hline $\mathrm{C} 48$ & 2.02648 & 1.29038 & 0.21431 \\
\hline
\end{tabular}




\begin{tabular}{|c|c|c|c|}
\hline C49 & 2.05032 & 1.28557 & 0.18811 \\
\hline $\mathrm{C} 50$ & 2.07151 & 1.30532 & 0.1851 \\
\hline C51 & 2.06956 & 1.33075 & 0.20792 \\
\hline C52 & 2.04577 & 1.336 & 0.23368 \\
\hline C53 & 2.02454 & 1.31618 & 0.23691 \\
\hline C54 & 2.00426 & 1.26723 & 0.21644 \\
\hline $\mathrm{C} 55$ & 2.17438 & 1.45748 & 0.16466 \\
\hline N56 & 2.15432 & 1.43684 & 0.17798 \\
\hline C57 & 2.01851 & 1.24091 & 0.23587 \\
\hline C58 & 2.01822 & 1.21508 & 0.21323 \\
\hline C59 & 2.03331 & 1.19235 & 0.22949 \\
\hline C60 & 2.04949 & 1.19461 & 0.26854 \\
\hline C61 & 2.04971 & 1.22007 & 0.2916 \\
\hline C62 & 2.03451 & 1.24281 & 0.27554 \\
\hline N63 & 2.14123 & 1.10413 & 0.34367 \\
\hline C64 & 2.15913 & 1.08279 & 0.36168 \\
\hline O65 & 1.94535 & 1.25218 & 0.2895 \\
\hline O66 & 1.99628 & 1.26296 & 0.1714 \\
\hline O67 & 1.98564 & 1.27899 & 0.28983 \\
\hline O68 & 1.93504 & 1.26817 & 0.17081 \\
\hline C69 & 1.73358 & 1.07874 & 0.14026 \\
\hline $\mathrm{C} 70$ & 1.7163 & 1.0578 & 0.12335 \\
\hline C71 & 1.72361 & 1.02968 & 0.12803 \\
\hline $\mathrm{C} 72$ & 1.74798 & 1.02279 & 0.15149 \\
\hline $\mathrm{C} 73$ & 1.76534 & 1.04375 & 0.16832 \\
\hline $\mathrm{C} 74$ & 2.19931 & 1.45045 & 0.14279 \\
\hline $\mathrm{C} 75$ & 2.21652 & 1.47123 & 0.12546 \\
\hline $\mathrm{C} 76$ & 2.20921 & 1.49938 & 0.12942 \\
\hline $\mathrm{C} 77$ & 2.18491 & 1.50647 & 0.15259 \\
\hline $\mathrm{C} 78$ & 2.16762 & 1.48567 & 0.16985 \\
\hline C79 & 2.1832 & 1.09115 & 0.38451 \\
\hline $\mathrm{C} 80$ & 2.20013 & 1.07165 & 0.40615 \\
\hline $\mathrm{C} 81$ & 2.19341 & 1.04325 & 0.40502 \\
\hline $\mathrm{C} 82$ & 2.1701 & 1.03459 & 0.38027 \\
\hline $\mathrm{C} 83$ & 2.15308 & 1.05413 & 0.35867 \\
\hline $\mathrm{C} 84$ & 1.74788 & 1.43927 & 0.38341 \\
\hline $\mathrm{C} 85$ & 1.73108 & 1.45876 & 0.4051 \\
\hline C86 & 1.73783 & 1.48716 & 0.40402 \\
\hline $\mathrm{C} 87$ & 1.76102 & 1.49582 & 0.37932 \\
\hline $\mathrm{C} 88$ & 1.77791 & 1.47628 & 0.35768 \\
\hline $\mathrm{C} 89$ & 1.72243 & 1.50753 & 0.43328 \\
\hline
\end{tabular}




\begin{tabular}{|c|c|c|c|}
\hline C90 & 1.8396 & 1.17862 & 0.20107 \\
\hline C91 & 1.86394 & 1.35982 & 0.28295 \\
\hline $\mathrm{C} 92$ & 2.09233 & 1.35159 & 0.20458 \\
\hline $\mathrm{C} 93$ & 2.06664 & 1.17071 & 0.28431 \\
\hline C94 & 1.31806 & 1.59966 & 0.20853 \\
\hline C95 & 2.1217 & 1.34253 & 0.2027 \\
\hline C96 & 2.14266 & 1.36215 & 0.19658 \\
\hline C97 & 2.1363 & 1.39054 & 0.19256 \\
\hline C98 & 2.10845 & 1.39933 & 0.19555 \\
\hline C99 & 2.08682 & 1.38019 & 0.20149 \\
\hline C100 & 2.05624 & 1.1434 & 0.28227 \\
\hline C101 & 2.07254 & 1.12089 & 0.29628 \\
\hline $\mathrm{C} 102$ & 2.09962 & 1.12512 & 0.31251 \\
\hline $\mathrm{C} 103$ & 2.1101 & 1.1524 & 0.31456 \\
\hline C104 & 2.09377 & 1.17493 & 0.30067 \\
\hline $\mathrm{C} 105$ & 1.83673 & 1.3556 & 0.29857 \\
\hline C106 & 1.82046 & 1.3781 & 0.31261 \\
\hline C107 & 1.8311 & 1.40533 & 0.31144 \\
\hline C108 & 1.85824 & 1.40956 & 0.29584 \\
\hline C109 & 1.87446 & 1.3871 & 0.2817 \\
\hline $\mathrm{C} 110$ & 1.84581 & 1.15009 & 0.19756 \\
\hline C111 & 1.82456 & 1.13047 & 0.19181 \\
\hline C112 & 1.79664 & 1.13899 & 0.18958 \\
\hline $\mathrm{C} 113$ & 1.79041 & 1.16736 & 0.19402 \\
\hline $\mathrm{C} 114$ & 1.8116 & 1.18694 & 0.1997 \\
\hline $\mathrm{C} 115$ & 2.1591 & 1.4104 & 0.18334 \\
\hline C116 & 1.77383 & 1.11905 & 0.18065 \\
\hline C117 & 1.47758 & 1.75498 & 0.28178 \\
\hline $\mathrm{C} 118$ & 1.4539 & 1.77646 & 0.28172 \\
\hline C119 & 1.46584 & 1.766 & 0.21067 \\
\hline $\mathrm{C} 120$ & 1.45138 & 1.74462 & 0.18052 \\
\hline $\mathrm{C} 121$ & 1.52518 & 1.73862 & 0.31501 \\
\hline C122 & 1.5221 & 1.71245 & 0.29383 \\
\hline $\mathrm{C} 123$ & 1.54281 & 1.69205 & 0.29737 \\
\hline $\mathrm{C} 124$ & 1.56728 & 1.69712 & 0.32182 \\
\hline $\mathrm{C} 125$ & 1.57046 & 1.72299 & 0.34299 \\
\hline $\mathrm{C} 126$ & 1.54984 & 1.7433 & 0.33966 \\
\hline $\mathrm{C} 127$ & 1.50341 & 1.76238 & 0.312 \\
\hline $\mathrm{C} 128$ & 1.67025 & 1.56956 & 0.37015 \\
\hline N129 & 1.64992 & 1.5898 & 0.35601 \\
\hline $\mathrm{C} 130$ & 1.51787 & 1.7888 & 0.29399 \\
\hline
\end{tabular}




\begin{tabular}{|c|c|c|c|}
\hline C131 & 1.53578 & 1.78713 & 0.25682 \\
\hline C132 & 1.55084 & 1.81031 & 0.24246 \\
\hline C133 & 1.54835 & 1.83596 & 0.2646 \\
\hline C134 & 1.52978 & 1.83806 & 0.30064 \\
\hline C135 & 1.51477 & 1.81485 & 0.31516 \\
\hline C136 & 1.48053 & 1.78781 & 0.18181 \\
\hline N137 & 1.64011 & 1.9277 & 0.19581 \\
\hline C138 & 1.65795 & 1.9489 & 0.17759 \\
\hline C139 & 1.40614 & 1.79256 & 0.31409 \\
\hline $\mathrm{C} 140$ & 1.38135 & 1.78763 & 0.33794 \\
\hline $\mathrm{C} 141$ & 1.36062 & 1.80784 & 0.34096 \\
\hline $\mathrm{C} 142$ & 1.36387 & 1.83386 & 0.32041 \\
\hline C143 & 1.38846 & 1.83918 & 0.29675 \\
\hline C144 & 1.40925 & 1.81887 & 0.29342 \\
\hline $\mathrm{C} 145$ & 1.42808 & 1.76895 & 0.31153 \\
\hline $\mathrm{C} 146$ & 1.26047 & 1.96067 & 0.37091 \\
\hline N147 & 1.28054 & 1.94039 & 0.35626 \\
\hline C148 & 1.41384 & 1.74238 & 0.29344 \\
\hline C149 & 1.41694 & 1.71645 & 0.31502 \\
\hline $\mathrm{C} 150$ & 1.4021 & 1.69311 & 0.30043 \\
\hline $\mathrm{C} 151$ & 1.38366 & 1.69495 & 0.26394 \\
\hline $\mathrm{C} 152$ & 1.38111 & 1.7205 & 0.24145 \\
\hline $\mathrm{C} 153$ & 1.39605 & 1.74381 & 0.25585 \\
\hline N154 & 1.29241 & 1.60235 & 0.19532 \\
\hline C155 & 1.27465 & 1.58091 & 0.17736 \\
\hline O156 & 1.48575 & 1.75207 & 0.2372 \\
\hline O157 & 1.43835 & 1.76455 & 0.35533 \\
\hline $\mathrm{O} 158$ & 1.44573 & 1.77951 & 0.23706 \\
\hline O159 & 1.49313 & 1.76693 & 0.3556 \\
\hline $\mathrm{C} 160$ & 1.69547 & 1.57726 & 0.39089 \\
\hline $\mathrm{C} 161$ & 1.71307 & 1.55701 & 0.40908 \\
\hline $\mathrm{C} 162$ & 1.7059 & 1.52874 & 0.40705 \\
\hline $\mathrm{C} 163$ & 1.68129 & 1.52096 & 0.38501 \\
\hline $\mathrm{C} 164$ & 1.66357 & 1.54124 & 0.367 \\
\hline $\mathrm{C} 165$ & 1.23544 & 1.95303 & 0.39193 \\
\hline C166 & 1.21805 & 1.97333 & 0.4103 \\
\hline $\mathrm{C} 167$ & 1.22528 & 2.00159 & 0.40821 \\
\hline $\mathrm{C} 168$ & 1.24977 & 2.00929 & 0.38609 \\
\hline $\mathrm{C} 169$ & 1.26725 & 1.98897 & 0.36785 \\
\hline $\mathrm{C} 170$ & 1.24992 & 1.58908 & 0.15627 \\
\hline C171 & 1.23308 & 1.56959 & 0.13449 \\
\hline
\end{tabular}




\begin{tabular}{|c|c|c|c|}
\hline C172 & 1.24056 & 1.54138 & 0.13379 \\
\hline $\mathrm{C} 173$ & 1.26451 & 1.53283 & 0.157 \\
\hline $\mathrm{C} 174$ & 1.28146 & 1.55238 & 0.17868 \\
\hline $\mathrm{C} 175$ & 1.68269 & 1.94045 & 0.15716 \\
\hline $\mathrm{C} 176$ & 1.69957 & 1.95964 & 0.13501 \\
\hline $\mathrm{C} 177$ & 1.69214 & 1.98785 & 0.13328 \\
\hline $\mathrm{C} 178$ & 1.6682 & 1.99672 & 0.15587 \\
\hline $\mathrm{C} 179$ & 1.6512 & 1.97746 & 0.17793 \\
\hline C180 & 1.70762 & 2.00756 & 0.103 \\
\hline C181 & 1.20893 & 2.02287 & 0.43434 \\
\hline C182 & 1.58951 & 1.67567 & 0.32549 \\
\hline $\mathrm{C} 183$ & 1.56546 & 1.86026 & 0.25067 \\
\hline $\mathrm{C} 184$ & 1.3415 & 1.85513 & 0.32379 \\
\hline C185 & 1.3667 & 1.67051 & 0.24999 \\
\hline C186 & 1.61447 & 1.9306 & 0.20861 \\
\hline H187 & 2.10648 & 1.08067 & 0.32724 \\
\hline H188 & 1.24042 & 1.51142 & 0.08103 \\
\hline H189 & 1.21024 & 1.53254 & 0.08187 \\
\hline H190 & 1.30612 & 1.82531 & 0.31632 \\
\hline H191 & 1.26971 & 1.8599 & 0.32915 \\
\hline H192 & 1.33168 & 1.92427 & 0.34285 \\
\hline H193 & 1.36885 & 1.89049 & 0.33179 \\
\hline H194 & 1.39977 & 1.64036 & 0.25858 \\
\hline H195 & 1.37168 & 1.60027 & 0.23603 \\
\hline H196 & 1.30119 & 1.65424 & 0.21497 \\
\hline H197 & 1.32905 & 1.69419 & 0.23813 \\
\hline H198 & 1.60305 & 1.83635 & 0.2401 \\
\hline H199 & 1.63112 & 1.876 & 0.2166 \\
\hline $\mathrm{H} 200$ & 1.56077 & 1.93038 & 0.23501 \\
\hline $\mathrm{H} 201$ & 1.53248 & 1.89059 & 0.25795 \\
\hline H202 & 1.56148 & 1.64084 & 0.33437 \\
\hline H203 & 1.59829 & 1.60626 & 0.34477 \\
\hline H204 & 1.66011 & 1.67044 & 0.32867 \\
\hline $\mathrm{H} 205$ & 1.62345 & 1.70487 & 0.31752 \\
\hline H206 & 1.25437 & 1.90652 & 0.34851 \\
\hline H207 & 1.67591 & 1.62377 & 0.34805 \\
\hline H208 & 1.82453 & 1.44967 & 0.32725 \\
\hline H209 & 1.96089 & 1.23475 & 0.23308 \\
\hline $\mathrm{H} 210$ & 1.97026 & 1.29631 & 0.23301 \\
\hline H211 & 1.98824 & 1.22712 & 0.32563 \\
\hline $\mathrm{H} 212$ & 1.96407 & 1.2348 & 0.36962 \\
\hline
\end{tabular}




\begin{tabular}{|c|c|c|c|}
\hline H213 & 1.99642 & 1.25382 & 0.36614 \\
\hline $\mathrm{H} 214$ & 1.92414 & 1.20966 & 0.25717 \\
\hline $\mathrm{H} 215$ & 1.88763 & 1.17554 & 0.25055 \\
\hline H216 & 1.84272 & 1.22937 & 0.16022 \\
\hline $\mathrm{H} 217$ & 1.87909 & 1.26425 & 0.16713 \\
\hline H218 & 1.89469 & 1.26823 & 0.2919 \\
\hline H219 & 1.86851 & 1.3078 & 0.31997 \\
\hline $\mathrm{H} 220$ & 1.89797 & 1.35778 & 0.21007 \\
\hline $\mathrm{H} 221$ & 1.92432 & 1.31906 & 0.18215 \\
\hline H222 & 1.93373 & 1.27838 & 0.36435 \\
\hline $\mathrm{H} 223$ & 1.94253 & 1.30461 & 0.32349 \\
\hline $\mathrm{H} 224$ & 1.96611 & 1.29732 & 0.36879 \\
\hline $\mathrm{H} 225$ & 2.05259 & 1.26621 & 0.16993 \\
\hline H226 & 2.08931 & 1.30076 & 0.16431 \\
\hline $\mathrm{H} 227$ & 2.04374 & 1.35532 & 0.25202 \\
\hline H228 & 2.00693 & 1.32148 & 0.25744 \\
\hline H229 & 2.00697 & 1.21208 & 0.18227 \\
\hline $\mathrm{H} 230$ & 2.03301 & 1.17315 & 0.21052 \\
\hline $\mathrm{H} 231$ & 2.06175 & 1.22247 & 0.32207 \\
\hline $\mathrm{H} 232$ & 2.03586 & 1.26224 & 0.29372 \\
\hline $\mathrm{H} 233$ & 1.98211 & 1.24812 & 0.1698 \\
\hline $\mathrm{H} 234$ & 1.94915 & 1.28307 & 0.16961 \\
\hline $\mathrm{H} 235$ & 1.72787 & 1.1001 & 0.13383 \\
\hline $\mathrm{H} 236$ & 1.69777 & 1.06363 & 0.10492 \\
\hline $\mathrm{H} 237$ & 1.75418 & 1.00124 & 0.15498 \\
\hline H238 & 1.7846 & 1.03807 & 0.18482 \\
\hline H239 & 2.20506 & 1.42904 & 0.13691 \\
\hline $\mathrm{H} 240$ & 2.23499 & 1.46523 & 0.10726 \\
\hline $\mathrm{H} 241$ & 2.17872 & 1.52805 & 0.15553 \\
\hline $\mathrm{H} 242$ & 2.14842 & 1.49149 & 0.18613 \\
\hline $\mathrm{H} 243$ & 2.18833 & 1.11304 & 0.38689 \\
\hline $\mathrm{H} 244$ & 2.21798 & 1.07887 & 0.42505 \\
\hline $\mathrm{H} 245$ & 2.16454 & 1.01277 & 0.37904 \\
\hline $\mathrm{H} 246$ & 2.13517 & 1.04658 & 0.34051 \\
\hline $\mathrm{H} 247$ & 1.74273 & 1.41738 & 0.38571 \\
\hline $\mathrm{H} 248$ & 1.71331 & 1.45155 & 0.42395 \\
\hline H249 & 1.76658 & 1.51763 & 0.37812 \\
\hline $\mathrm{H} 250$ & 1.79572 & 1.48383 & 0.33948 \\
\hline $\mathrm{H} 251$ & 1.73782 & 1.518 & 0.45547 \\
\hline $\mathrm{H} 252$ & 1.70797 & 1.49641 & 0.45606 \\
\hline $\mathrm{H} 253$ & 1.3285 & 1.57979 & 0.20662 \\
\hline
\end{tabular}




\begin{tabular}{|c|c|c|c|}
\hline H254 & 2.12716 & 1.32079 & 0.20615 \\
\hline $\mathrm{H} 255$ & 2.16399 & 1.3551 & 0.19454 \\
\hline H256 & 2.10327 & 1.42116 & 0.19238 \\
\hline $\mathrm{H} 257$ & 2.06566 & 1.38779 & 0.20224 \\
\hline H258 & 2.03535 & 1.13944 & 0.27032 \\
\hline H259 & 2.06391 & 1.10011 & 0.29457 \\
\hline $\mathrm{H} 260$ & 2.1309 & 1.15636 & 0.32693 \\
\hline H261 & 2.10255 & 1.19565 & 0.30206 \\
\hline H262 & 1.82784 & 1.33491 & 0.29931 \\
\hline $\mathrm{H} 263$ & 1.7996 & 1.37414 & 0.32442 \\
\hline H264 & 1.86698 & 1.43031 & 0.29476 \\
\hline $\mathrm{H} 265$ & 1.89541 & 1.39105 & 0.27029 \\
\hline H266 & 1.86715 & 1.143 & 0.19804 \\
\hline H267 & 1.83005 & 1.10874 & 0.18833 \\
\hline H268 & 1.76907 & 1.17446 & 0.19252 \\
\hline H269 & 1.80604 & 1.20866 & 0.20305 \\
\hline $\mathrm{H} 270$ & 2.17992 & 1.4022 & 0.18011 \\
\hline $\mathrm{H} 271$ & 1.75295 & 1.12716 & 0.1776 \\
\hline $\mathrm{H} 272$ & 1.46911 & 1.73476 & 0.29316 \\
\hline $\mathrm{H} 273$ & 1.46237 & 1.79666 & 0.29338 \\
\hline $\mathrm{H} 274$ & 1.44213 & 1.72786 & 0.20127 \\
\hline $\mathrm{H} 275$ & 1.46652 & 1.73496 & 0.15723 \\
\hline $\mathrm{H} 276$ & 1.43457 & 1.75466 & 0.16067 \\
\hline $\mathrm{H} 277$ & 1.504 & 1.70733 & 0.27406 \\
\hline $\mathrm{H} 278$ & 1.53986 & 1.67241 & 0.28025 \\
\hline $\mathrm{H} 279$ & 1.58889 & 1.7275 & 0.3626 \\
\hline $\mathrm{H} 280$ & 1.55345 & 1.76306 & 0.35608 \\
\hline $\mathrm{H} 281$ & 1.53859 & 1.76761 & 0.23933 \\
\hline $\mathrm{H} 282$ & 1.56452 & 1.80811 & 0.21397 \\
\hline $\mathrm{H} 283$ & 1.5274 & 1.85752 & 0.31849 \\
\hline $\mathrm{H} 284$ & 1.50093 & 1.81738 & 0.34333 \\
\hline $\mathrm{H} 285$ & 1.46552 & 1.79796 & 0.15863 \\
\hline $\mathrm{H} 286$ & 1.49736 & 1.77803 & 0.16188 \\
\hline H287 & 1.48979 & 1.80416 & 0.20349 \\
\hline $\mathrm{H} 288$ & 1.37775 & 1.76777 & 0.35398 \\
\hline H289 & 1.34209 & 1.80312 & 0.35993 \\
\hline $\mathrm{H} 290$ & 1.39145 & 1.85894 & 0.28005 \\
\hline $\mathrm{H} 291$ & 1.42745 & 1.82417 & 0.27427 \\
\hline $\mathrm{H} 292$ & 1.43065 & 1.71412 & 0.34359 \\
\hline $\mathrm{H} 293$ & 1.40448 & 1.67375 & 0.31859 \\
\hline $\mathrm{H} 294$ & 1.36747 & 1.72252 & 0.2127 \\
\hline
\end{tabular}




\begin{tabular}{|c|c|c|c|}
\hline H295 & 1.39317 & 1.76324 & 0.23811 \\
\hline H296 & 1.42283 & 1.75913 & 0.37527 \\
\hline H297 & 1.5086 & 1.77255 & 0.37569 \\
\hline H298 & 1.70112 & 1.59885 & 0.39522 \\
\hline H299 & 1.73179 & 1.56355 & 0.42636 \\
\hline H300 & 1.67523 & 1.49928 & 0.38351 \\
\hline H301 & 1.64416 & 1.53491 & 0.35162 \\
\hline H302 & 1.22976 & 1.93146 & 0.39628 \\
\hline H303 & 1.19944 & 1.96685 & 0.42769 \\
\hline H304 & 1.25592 & 2.03095 & 0.38464 \\
\hline H305 & 1.28655 & 1.99524 & 0.35231 \\
\hline H306 & 1.24423 & 1.61087 & 0.15525 \\
\hline H307 & 1.21471 & 1.57671 & 0.11683 \\
\hline H308 & 1.27061 & 1.51115 & 0.15684 \\
\hline H309 & 1.29992 & 1.545 & 0.19558 \\
\hline H310 & 1.68835 & 1.91864 & 0.15691 \\
\hline H311 & 1.71795 & 1.95229 & 0.11787 \\
\hline H312 & 1.66214 & 2.01841 & 0.15494 \\
\hline H313 & 1.63274 & 1.98508 & 0.19431 \\
\hline H314 & 1.69237 & 2.01718 & 0.07959 \\
\hline H315 & 1.72247 & 1.99598 & 0.08149 \\
\hline H316 & 1.22353 & 2.03397 & 0.45712 \\
\hline H317 & 1.19363 & 2.01245 & 0.45653 \\
\hline H318 & 1.6041 & 1.95049 & 0.20615 \\
\hline
\end{tabular}

\section{Figure S14. Spectra data of the catalysis}

\subsection{NMR and HPLC of the secondary alcohols obtained with CCOF-1/Ti}<smiles>CCC(O)c1ccccc1</smiles>

(S)-1-phenylpropan-1-ol: Optical purity is determined by HPLC with a ChiralCel OD-H column: $25{ }^{\circ} \mathrm{C}$; hexane $/ \mathrm{PrOH}=98 / 2$; flow rate $=1.0 \mathrm{~mL} / \mathrm{min}$; $\mathrm{t}_{\text {major }}=17.28 \mathrm{~min}, \mathrm{t}_{\text {minor }}=14.92 \mathrm{~min}$; ee $=90 \% .{ }^{1} \mathrm{H} \mathrm{NMR}\left(400 \mathrm{MHz}, \mathrm{CDCl}_{3}\right) \delta 7.35-$ $7.25(\mathrm{~m}, 5 \mathrm{H}), 4.59$ (td, $J=6.7,3.1 \mathrm{~Hz}, 1 \mathrm{H}), 1.92(\mathrm{~s}, 1 \mathrm{H}), 1.87-1.68(\mathrm{~m}, 2 \mathrm{H}), 0.91$ (t, $J=7.4 \mathrm{~Hz}, 3 \mathrm{H})$. 


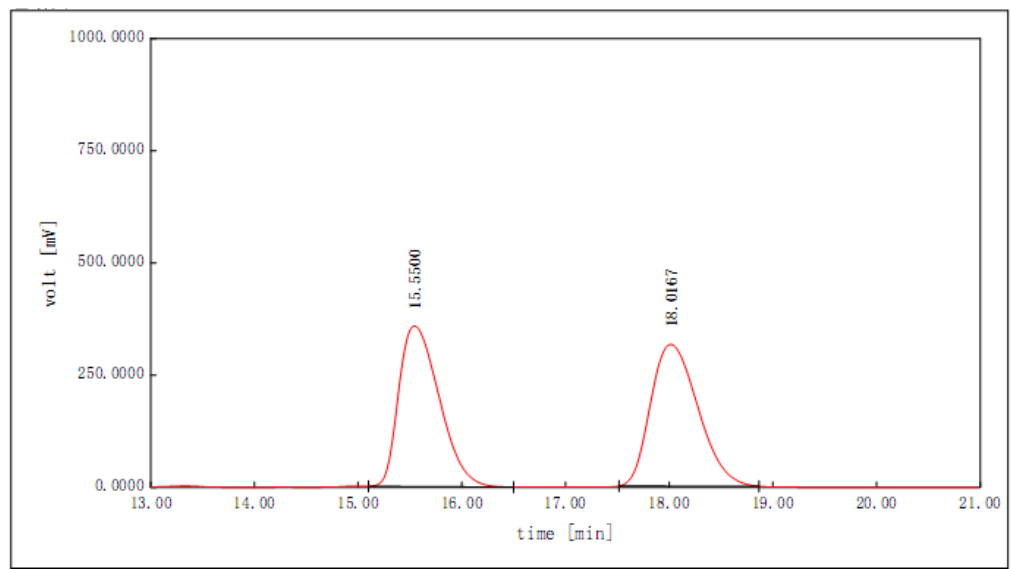

\begin{tabular}{|l|l|l|l|l|}
\hline Serial Number & Retention Time [min] & Area [mAbs*s] & Type & Area \% \\
\hline 1 & 15.5500 & 9691.5263 & BB & 49.2004 \\
\hline 2 & 18.0167 & 10006.5303 & BB & 50.7996 \\
\hline The Total & & 8497.1375 & & \\
\hline
\end{tabular}

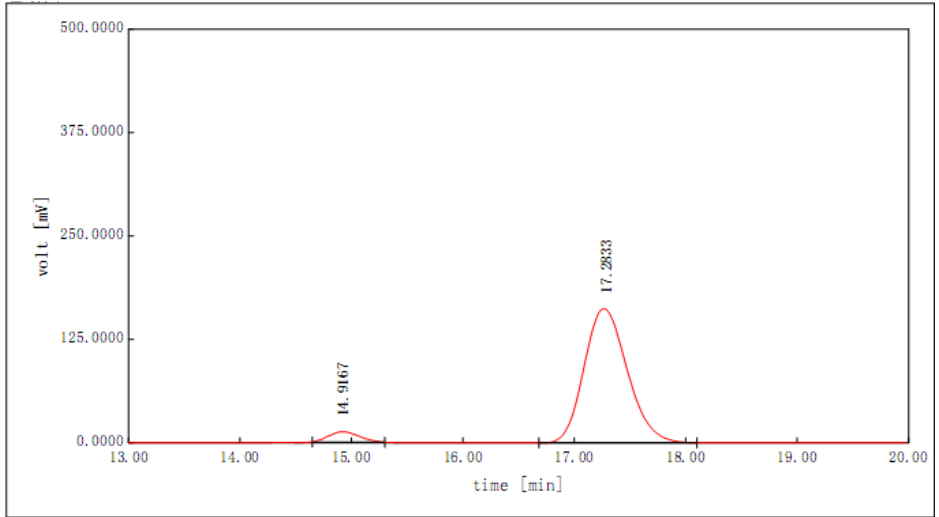

\begin{tabular}{|l|l|l|l|l|}
\hline Serial Number & Retention Time [min] & Area [mAbs*s] & Type & Area \% \\
\hline 1 & 14.9167 & 237.1649 & BB & 5.1259 \\
\hline 2 & 17.2833 & 4389.6497 & BB & 94.8741 \\
\hline The Total & & 4626.8146 & & \\
\hline
\end{tabular}

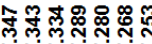

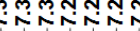

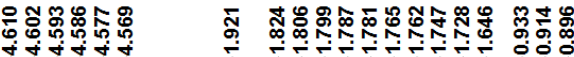

ఠ

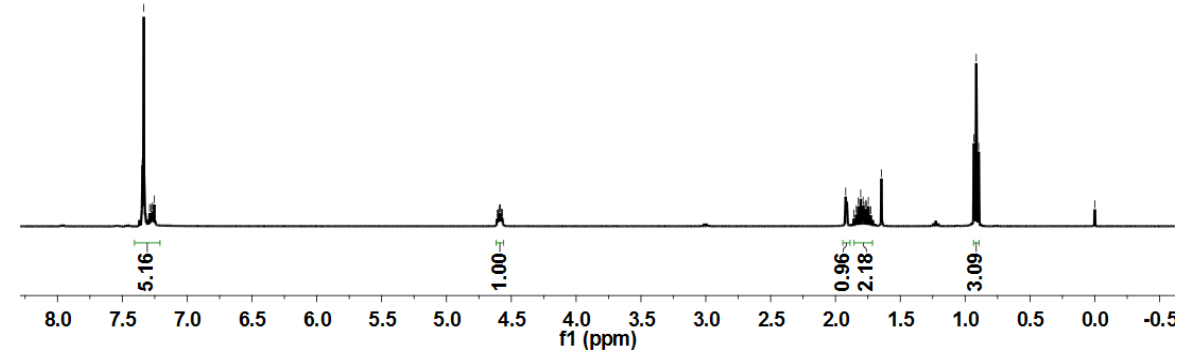


(R)-1-phenylpropan-1-ol: Optical purity is determined by HPLC with a ChiralCel OD-H column: $25{ }^{\circ} \mathrm{C}$; hexane/ $\mathrm{PrOH}=98 / 2$; flow rate $=1.0 \mathrm{~mL} / \mathrm{min}$; $\mathrm{t}_{\text {major }}=14.90 \mathrm{~min}, \mathrm{t}_{\text {minor }}=17.62 \mathrm{~min}$; ee $=90 \%$.

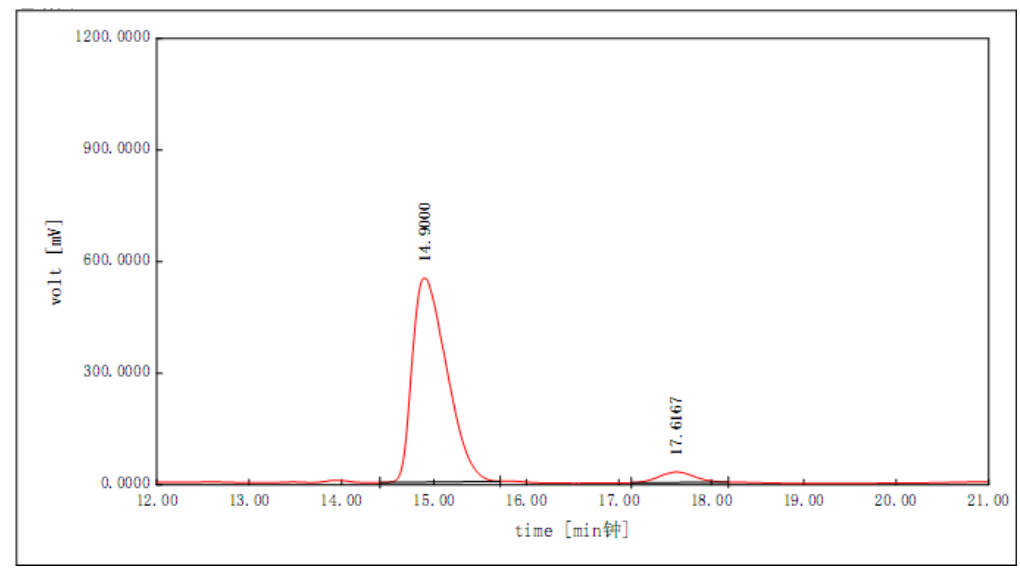

\begin{tabular}{|l|l|l|l|l|}
\hline Serial Number & Retention Time $[\mathrm{min}]$ & Area $[\mathrm{mAbs}$ s] & Type & Area \% \\
\hline 1 & 14.9000 & 14210.0772 & BB & 95.0076 \\
\hline 2 & 17.6167 & 746.7046 & BB & 4.9924 \\
\hline The Total & & 14956.7818 & & \\
\hline
\end{tabular}

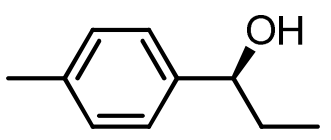

(S)-1-(p-tolyl)propan-1-ol: Optical purity is determined by HPLC with a ChiralCel AD-H column: $25{ }^{\circ} \mathrm{C}$; hexane/iPrOH $=98 / 2$; flow rate $=1.0$ $\mathrm{mL} / \mathrm{min} ; \mathrm{t}_{\text {major }}=17.70 \mathrm{~min}, \mathrm{t}_{\text {minor }}=15.32 \mathrm{~min}$; ee $=93 \% .{ }^{1} \mathrm{H} \mathrm{NMR}\left(400 \mathrm{MHz}, \mathrm{CDCl}_{3}\right)$ $\delta 7.23(\mathrm{~d}, J=8.1 \mathrm{~Hz}, 2 \mathrm{H}), 7.16(\mathrm{~d}, J=7.8 \mathrm{~Hz}, 2 \mathrm{H}), 4.56$ (t, $J=6.6 \mathrm{~Hz}, 1 \mathrm{H}), 2.35$ (s, $3 \mathrm{H}), 1.86-1.70(\mathrm{~m}, 3 \mathrm{H}), 0.91(\mathrm{t}, J=7.4 \mathrm{~Hz}, 3 \mathrm{H})$.

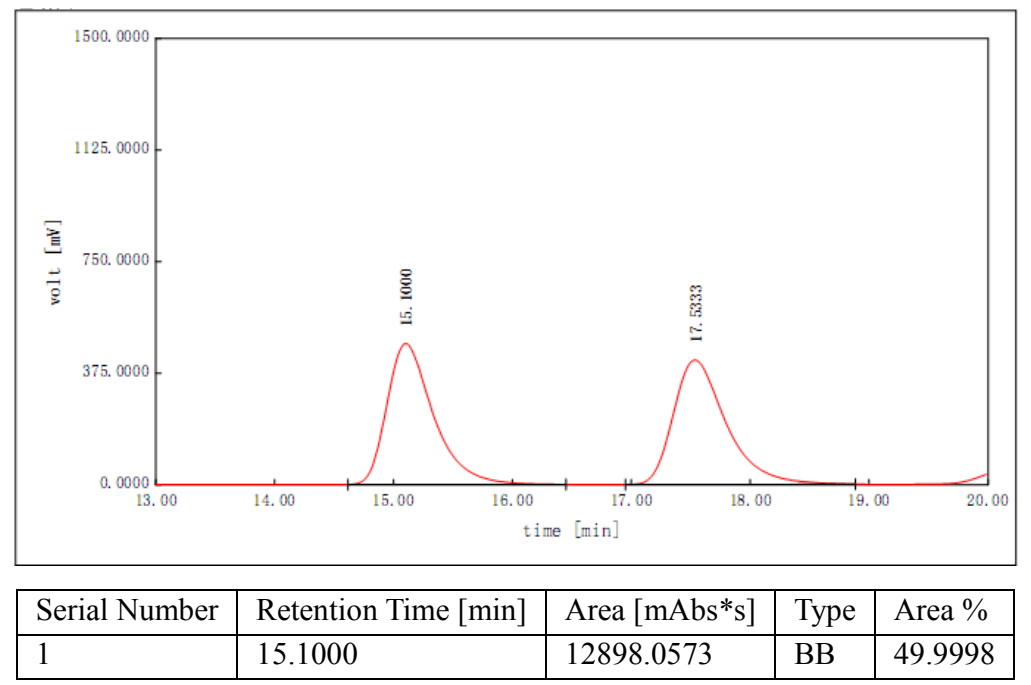




\begin{tabular}{|l|l|l|l|l|}
\hline 2 & 17.5333 & 12898.1789 & BB & 50.0002 \\
\hline The Total & & 25796.2361 & & \\
\hline
\end{tabular}

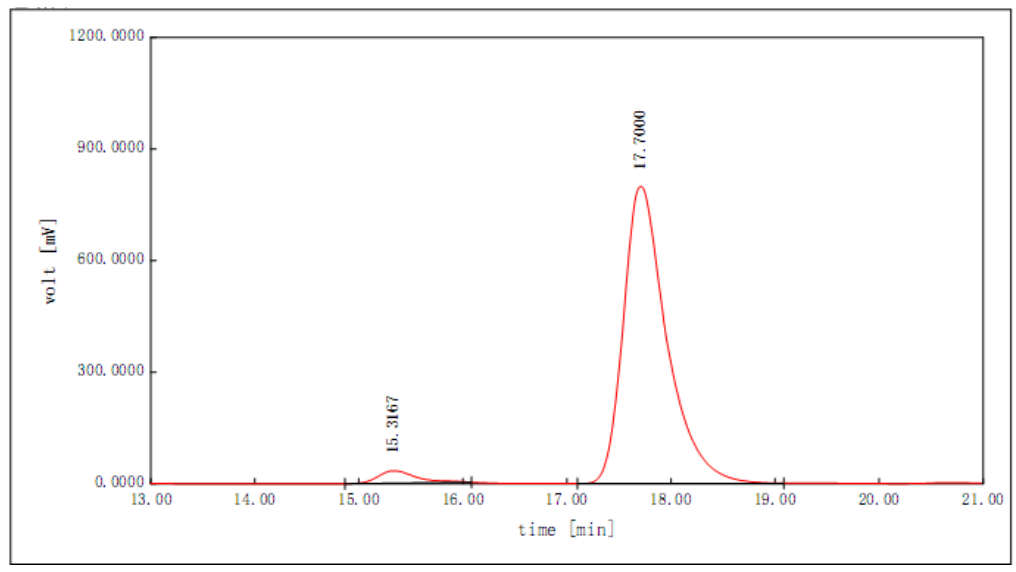

\begin{tabular}{|l|l|l|l|l|}
\hline Serial Number & Retention Time [min] & Area [mAbs*s] & Type & Area \% \\
\hline 1 & 15.3167 & 843.6958 & BB & 3.5252 \\
\hline 2 & 17.7000 & 23089.6985 & BB & 96.4748 \\
\hline The Total & & 23933.3943 & & \\
\hline
\end{tabular}

ำ

ñNiñ

ํㅜㅇํํำ

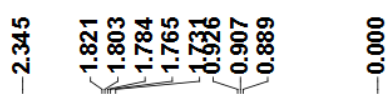

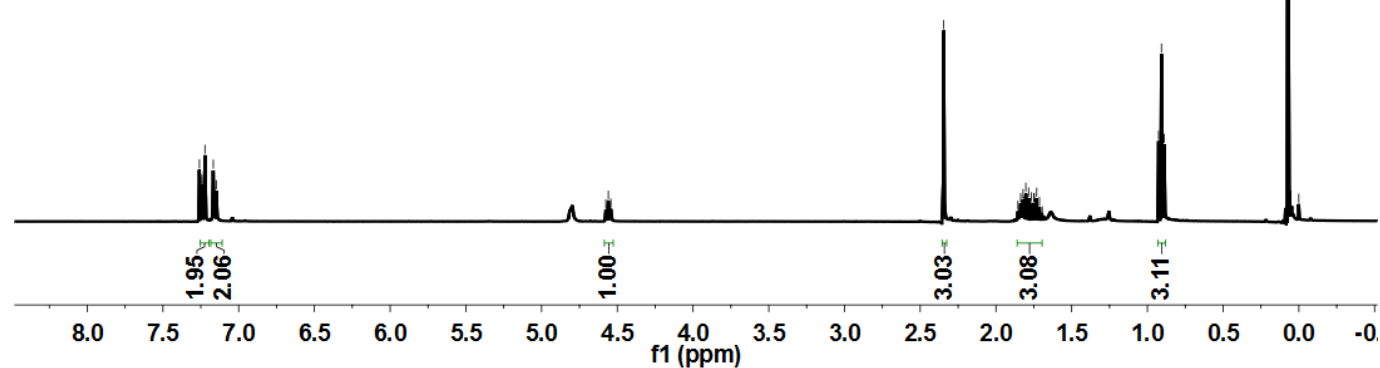<smiles>CCC(O)c1ccc(Cl)cc1</smiles>

(S)-1-(4-chlorophenyl)propan-1-ol: Optical purity is determined by HPLC with a ChiralCel OD-H column: $25{ }^{\circ} \mathrm{C}$; hexane/iPrOH $=98 / 2$; flow rate $=1.0 \mathrm{~mL} / \mathrm{min} ; \mathrm{t}_{\text {major }}=12.65 \mathrm{~min}, \mathrm{t}_{\text {minor }}=13.97 \mathrm{~min}$; ee $=90 \%$. ${ }^{1} \mathrm{H} \mathrm{NMR}$ $\left(400 \mathrm{MHz}, \mathrm{CDCl}_{3}\right) \delta 7.33-7.26(\mathrm{~m}, 4 \mathrm{H}), 4.59$ (t, $\left.J=6.5 \mathrm{~Hz}, 1 \mathrm{H}\right), 1.91(\mathrm{~s}, 1 \mathrm{H}), 1.81$ 
$-1.72(\mathrm{~m}, 2 \mathrm{H}), 0.90(\mathrm{t}, J=7.4 \mathrm{~Hz}, 3 \mathrm{H})$.

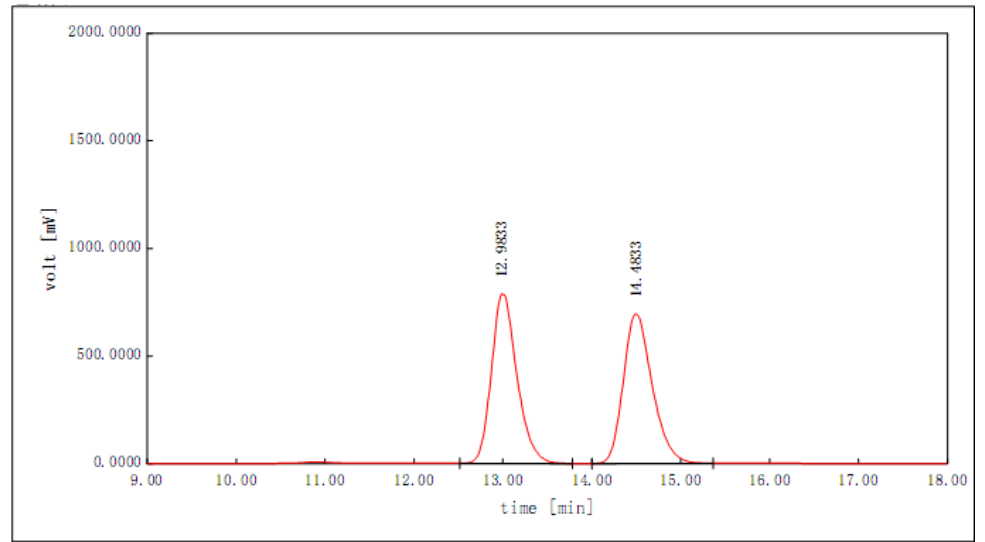

\begin{tabular}{|l|l|l|l|l|}
\hline Serial Number & Retention Time [min] & Area [mAbs*s] & Type & Area \% \\
\hline 1 & 12.9833 & 15704.6761 & BB & 50.0233 \\
\hline 2 & 14.4833 & 15690.0514 & BB & 49.9767 \\
\hline The Total & & 31394.7275 & & \\
\hline
\end{tabular}

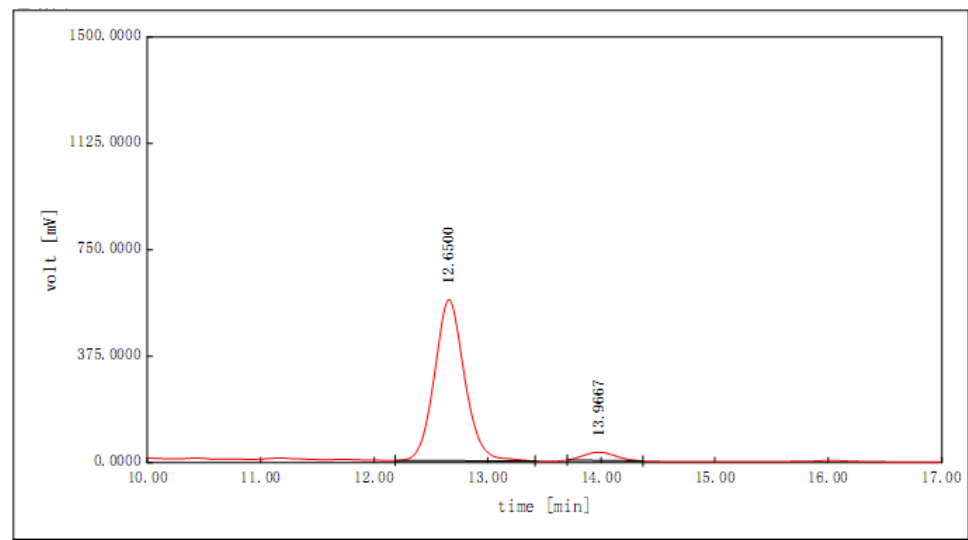

\begin{tabular}{|l|l|l|l|l|}
\hline Serial Number & Retention Time $[\mathrm{min}]$ & Area $[\mathrm{mAbs*}$ s] & Type & Area \% \\
\hline 1 & 12.6500 & 10436.2973 & BB & 94.9333 \\
\hline 2 & 13.9667 & 556.9969 & BB & 5.0667 \\
\hline The Total & & 10993.2942 & & \\
\hline
\end{tabular}




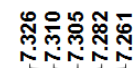
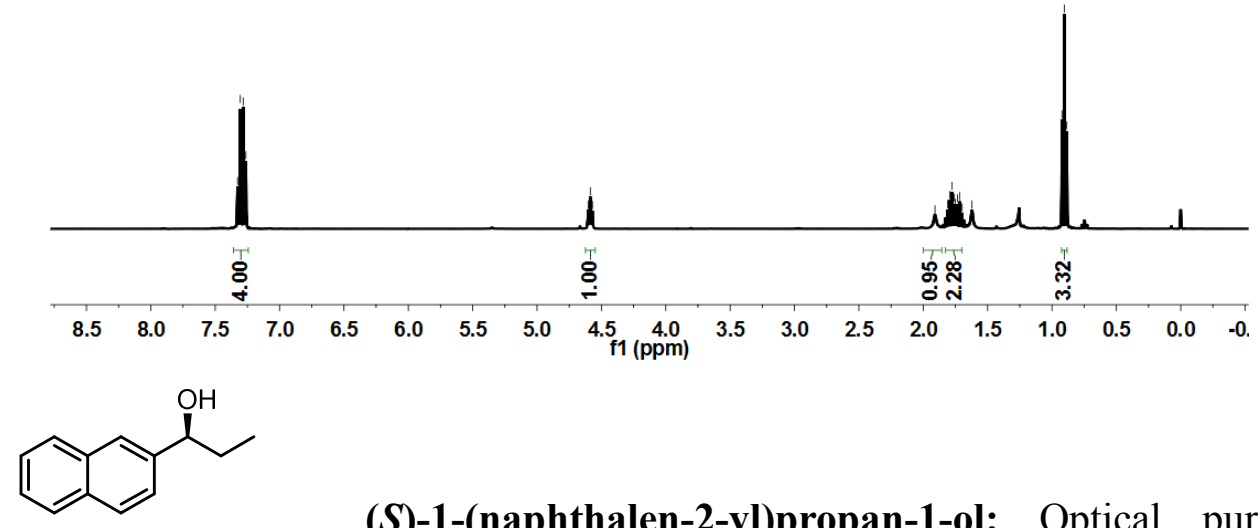

(S)-1-(naphthalen-2-yl)propan-1-ol: Optical purity is determined by HPLC with a ChiralCel OD-H column: $25^{\circ} \mathrm{C}$; hexane/iPrOH $=90 / 10$; flow rate $=0.5 \mathrm{~mL} / \mathrm{min} ; \mathrm{t}_{\text {major }}=20.02 \mathrm{~min}, \mathrm{t}_{\text {minor }}=21.95 \mathrm{~min}$; ee $=94 \%$. ${ }^{1} \mathrm{H} \mathrm{NMR}$ $\left(400 \mathrm{MHz}, \mathrm{CDCl}_{3}\right) \delta 7.83(\mathrm{dd}, J=7.0,2.7 \mathrm{~Hz}, 3 \mathrm{H}), 7.77(\mathrm{~s}, 1 \mathrm{H}), 7.48-7.46(\mathrm{~m}, 3 \mathrm{H})$, $4.77(\mathrm{t}, J=6.6 \mathrm{~Hz}, 1 \mathrm{H}), 2.02(\mathrm{~s}, 1 \mathrm{H}), 1.91-1.84(\mathrm{~m}, 2 \mathrm{H}), 0.94(\mathrm{t}, J=7.4 \mathrm{~Hz}, 3 \mathrm{H})$.

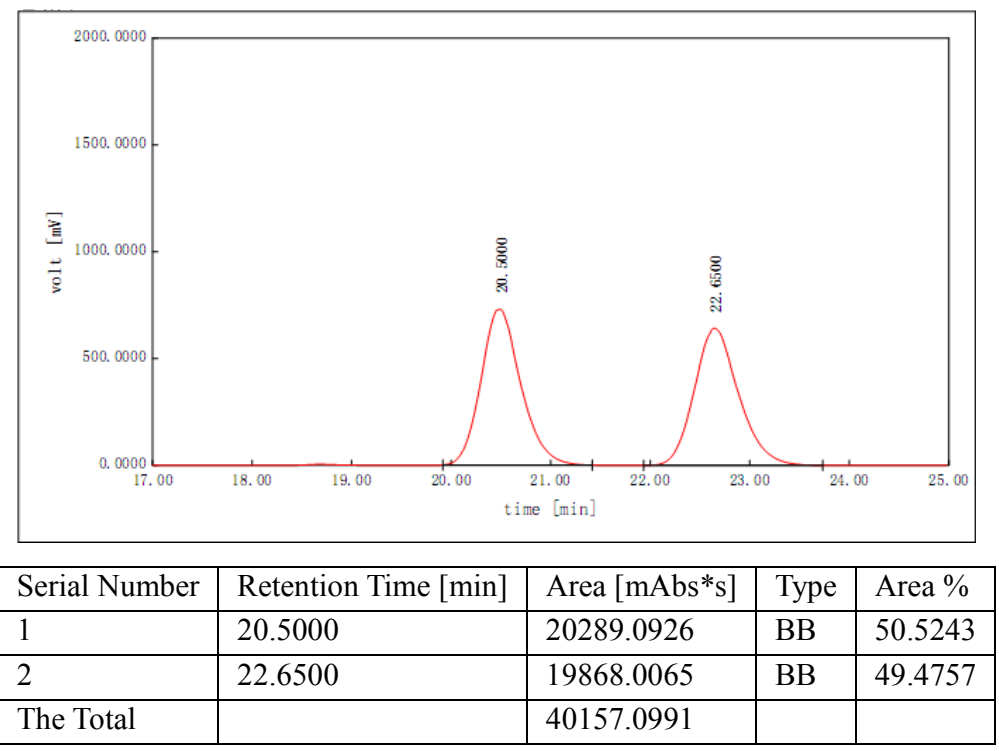




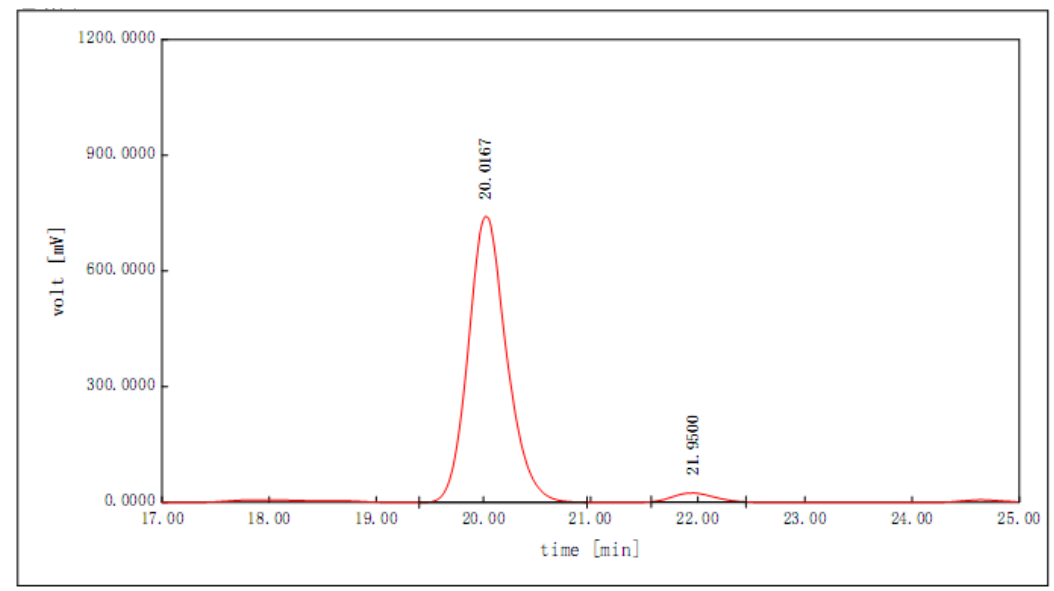

\begin{tabular}{|c|c|c|c|c|}
\hline Serial Number & Retention Time [min] & Area [mAbs*s] & Type & Area \% \\
\hline 1 & 20.0167 & 18487.3624 & $\mathrm{BB}$ & 96.9210 \\
\hline 2 & 21.9500 & 587.3095 & $\mathrm{BB}$ & 3.0790 \\
\hline The Total & & 19074.6719 & & \\
\hline 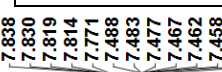 & 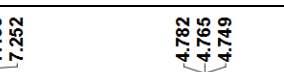 & 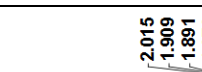 & & $\begin{array}{l}8 \\
0 \\
\end{array}$ \\
\hline
\end{tabular}
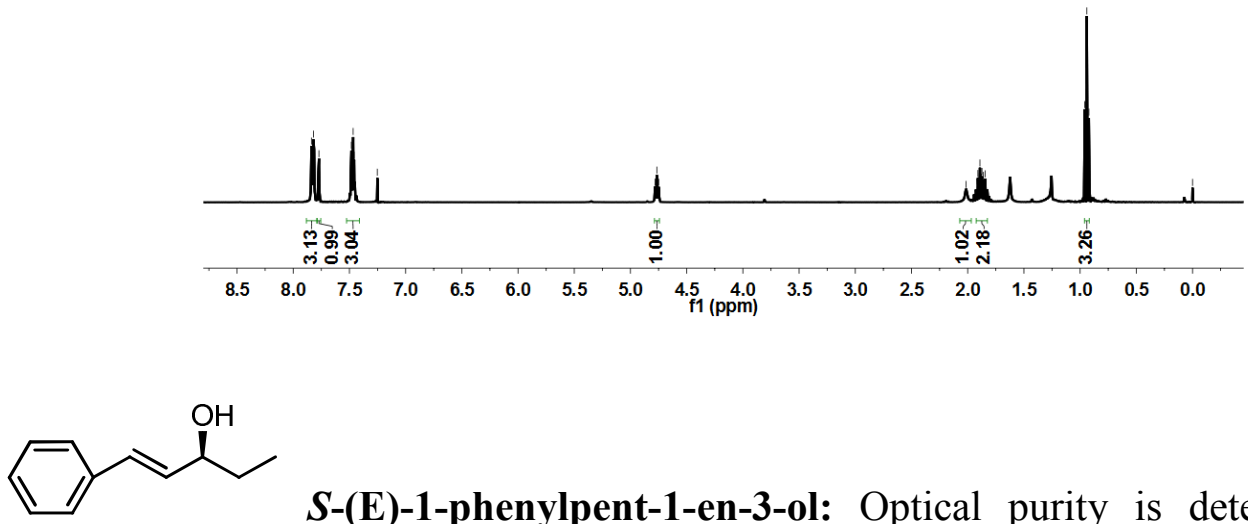

$\boldsymbol{S}$-(E)-1-phenylpent-1-en-3-ol: Optical purity is determined by HPLC with a ChiralCel OD-H column: $25{ }^{\circ} \mathrm{C}$; hexane/iPrOH $=95 / 5$; flow rate $=1.0$ $\mathrm{mL} / \mathrm{min} ; \mathrm{t}_{\text {major }}=22.85 \mathrm{~min}, \mathrm{t}_{\text {minor }}=13.67 \mathrm{~min}$; ee $=91 \% .{ }^{1} \mathrm{H} \mathrm{NMR}\left(400 \mathrm{MHz}, \mathrm{CDCl}_{3}\right)$ $\delta 7.39-7.22(\mathrm{~m}, 5 \mathrm{H}), 6.57(\mathrm{~d}, J=15.9 \mathrm{~Hz}, 1 \mathrm{H}), 6.21(\mathrm{dd}, J=15.9,6.8 \mathrm{~Hz}, 1 \mathrm{H}), 4.20$ $(\mathrm{q}, J=6.5 \mathrm{~Hz}, 1 \mathrm{H}), 1.98(\mathrm{~s}, 1 \mathrm{H}), 1.72-1.60(\mathrm{~m}, 2 \mathrm{H}), 0.96(\mathrm{t}, J=7.4 \mathrm{~Hz}, 3 \mathrm{H})$. 


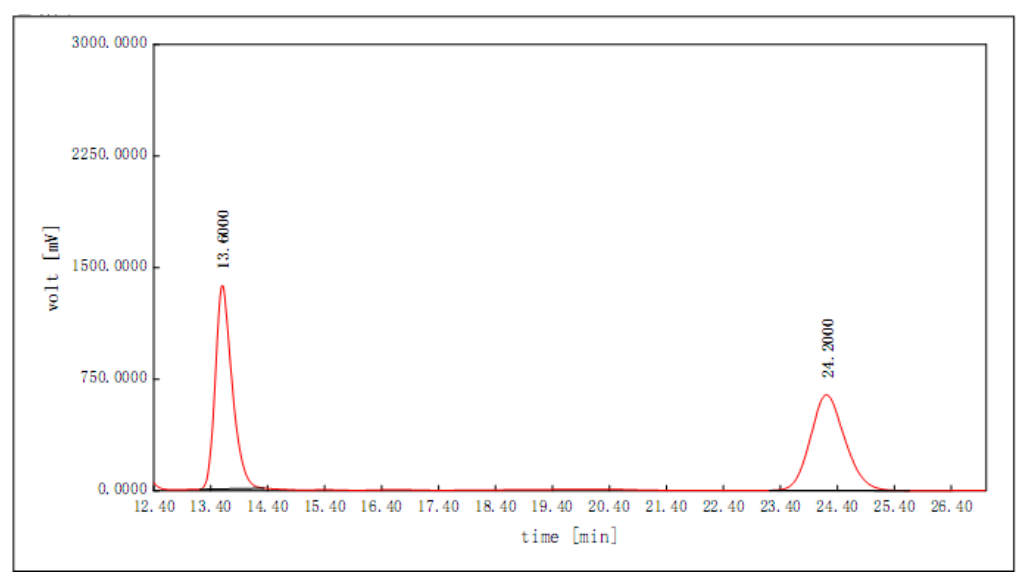

\begin{tabular}{|l|l|l|l|l|}
\hline Serial Number & Retention Time $[\mathrm{min}]$ & Area $[\mathrm{mAbs}$ *s] & Type & Area \% \\
\hline 1 & 13.6000 & 27983.3421 & BB & 50.7867 \\
\hline 2 & 24.2000 & 27116.4005 & BB & 49.2133 \\
\hline The Total & & 55099.7426 & & \\
\hline
\end{tabular}

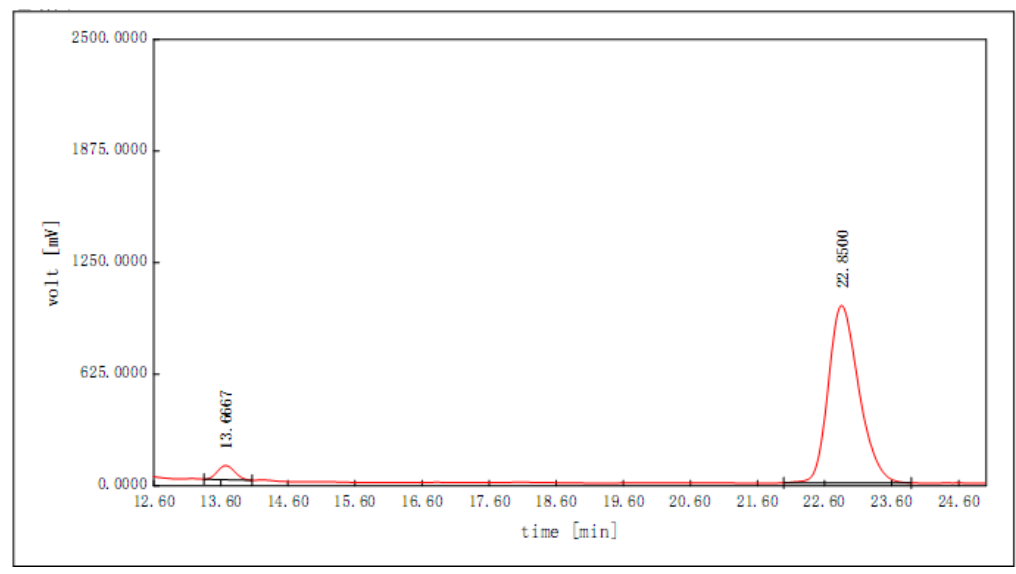

\begin{tabular}{|l|l|l|l|l|}
\hline Serial Number & Retention Time [min] & Area [mAbs*s] & Type & Area \% \\
\hline 1 & 13.6667 & 1410.7564 & BB & 4.2908 \\
\hline 2 & 22.8500 & 31468.2392 & BB & 95.7092 \\
\hline The Total & & 32878.9957 & & \\
\hline
\end{tabular}

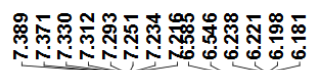

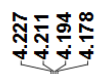

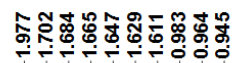

¿.

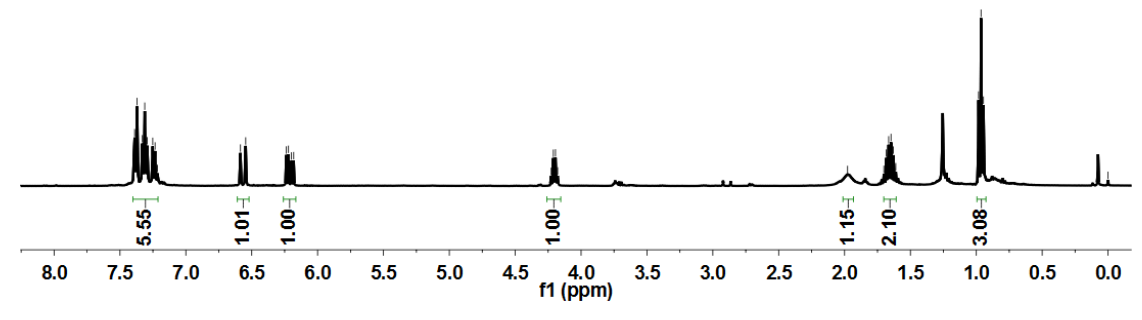


<smiles>CCC(O)c1cc2cccc3ccc4cccc1c4c32</smiles>

(S)-1-(pyren-4-yl)propan-1-ol: Optical purity is determined by HPLC with a ChiralCel OD-H column: $25{ }^{\circ} \mathrm{C}$; hexane/iPrOH $=90 / 10$; flow rate $=1.0$ $\mathrm{mL} / \mathrm{min} ; \mathrm{t}_{\text {major }}=14.70 \mathrm{~min}, \mathrm{t}_{\text {minor }}=17.20 \mathrm{~min}$; ee $=82.0 \% .{ }^{1} \mathrm{H}$ NMR $(400 \mathrm{MHz}$, $\left.\mathrm{CDCl}_{3}\right) \delta 8.32(\mathrm{~d}, J=9.3 \mathrm{~Hz}, 1 \mathrm{H}), 8.18-8.16(\mathrm{~m}, 4 \mathrm{H}), 8.08(\mathrm{~d}, J=9.4 \mathrm{~Hz}, 1 \mathrm{H}), 8.03$ $-7.97(\mathrm{~m}, 3 \mathrm{H}), 5.69$ (t, $J=6.4 \mathrm{~Hz}, 1 \mathrm{H}), 2.17(\mathrm{~s}, 1 \mathrm{H}), 2.11-2.03(\mathrm{~m}, 2 \mathrm{H}), 1.04(\mathrm{t}, J=$ $7.4 \mathrm{~Hz}, 3 \mathrm{H})$.

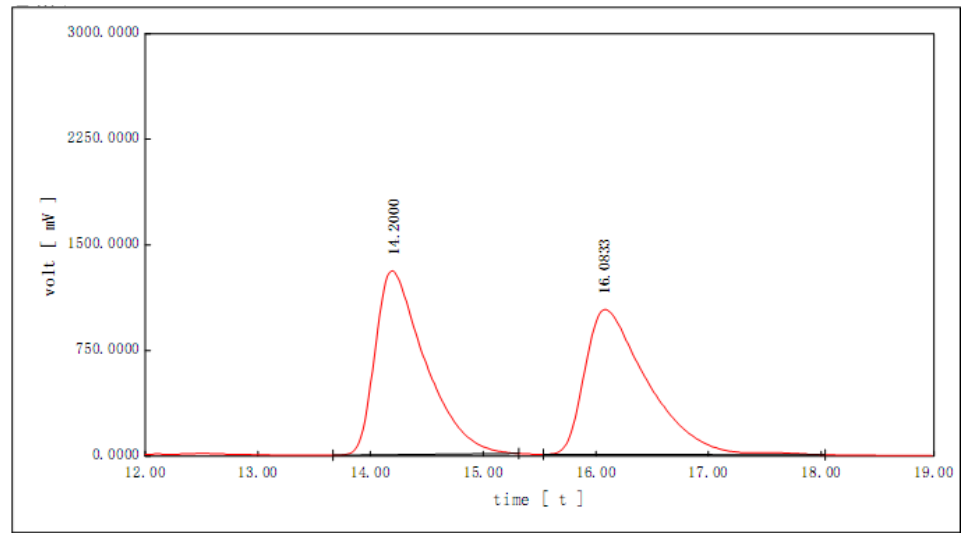

\begin{tabular}{|l|l|l|l|l|}
\hline Serial Number & Retention Time [min] & Area [mAbs*s] & Type & Area \% \\
\hline 1 & 14.2000 & 40143.5378 & BB & 50.2440 \\
\hline 2 & 16.0833 & 39753.6011 & BB & 49.7560 \\
\hline The Total & & 79897.1389 & & \\
\hline
\end{tabular}

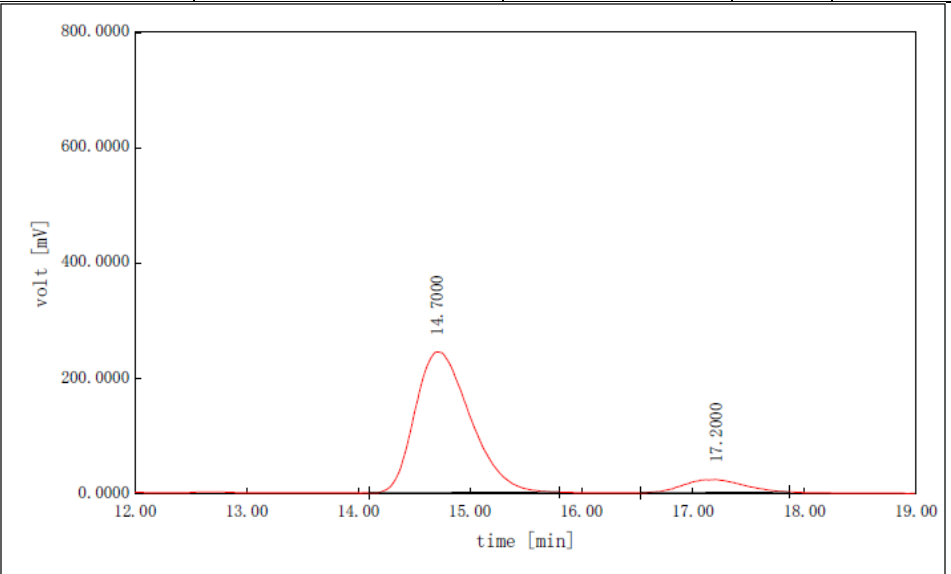

\begin{tabular}{|l|l|l|l|l|}
\hline Serial Number & Retention Time [min] & Area [mAbs*s] & Type & Area \% \\
\hline 1 & 14.7000 & 8372.4325 & BB & 90.9685 \\
\hline 2 & 17.2000 & 831.2337 & BV & 9.0315 \\
\hline The Total & & 9203.6661 & & \\
\hline
\end{tabular}




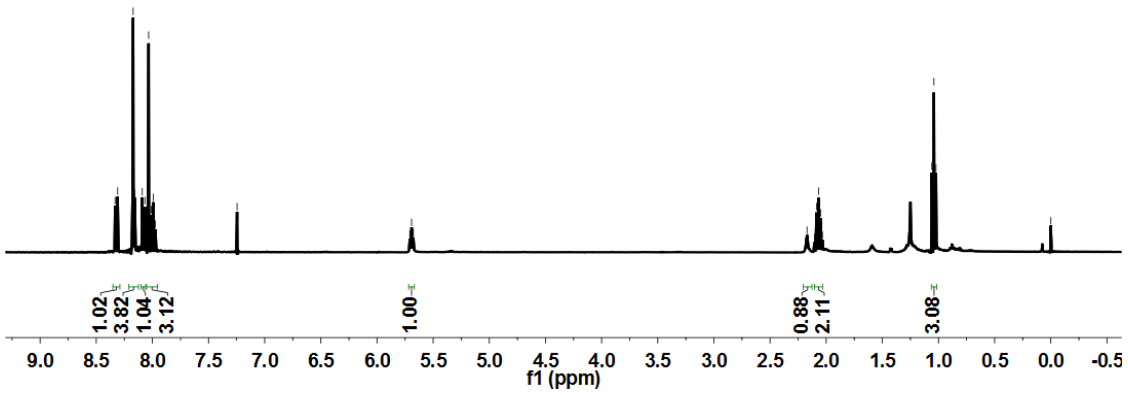<smiles></smiles>

1-(coronen-1-yl)propan-1-ol: ${ }^{1} \mathrm{H}$ NMR (400 MHz, DMSO) $\delta 9.25(\mathrm{~d}$, $J=9.2 \mathrm{~Hz}, 1 \mathrm{H}), 9.15(\mathrm{~s}, 1 \mathrm{H}), 9.01-8.96(\mathrm{~m}, 9 \mathrm{H}), 6.02-5.98(\mathrm{~m}, 1 \mathrm{H}), 5.78(\mathrm{~d}, J=$ $4.0 \mathrm{~Hz}, 1 \mathrm{H}), 2.32-2.29(\mathrm{~m}, 1 \mathrm{H}), 2.19-2.12(\mathrm{~m}, 1 \mathrm{H}), 1.18(\mathrm{t}, J=7.2 \mathrm{~Hz}, 3 \mathrm{H}) .{ }^{13} \mathrm{C}$ NMR (101 MHz, DMSO) $\delta 141.40,129.07,128.96,128.80,128.72,128.65,126.87$, $126.57,126.52$, 123.94, 122.96, 122.67, 122.48, 122.31, 121.71, 72.47, 32.75, 11.50 .

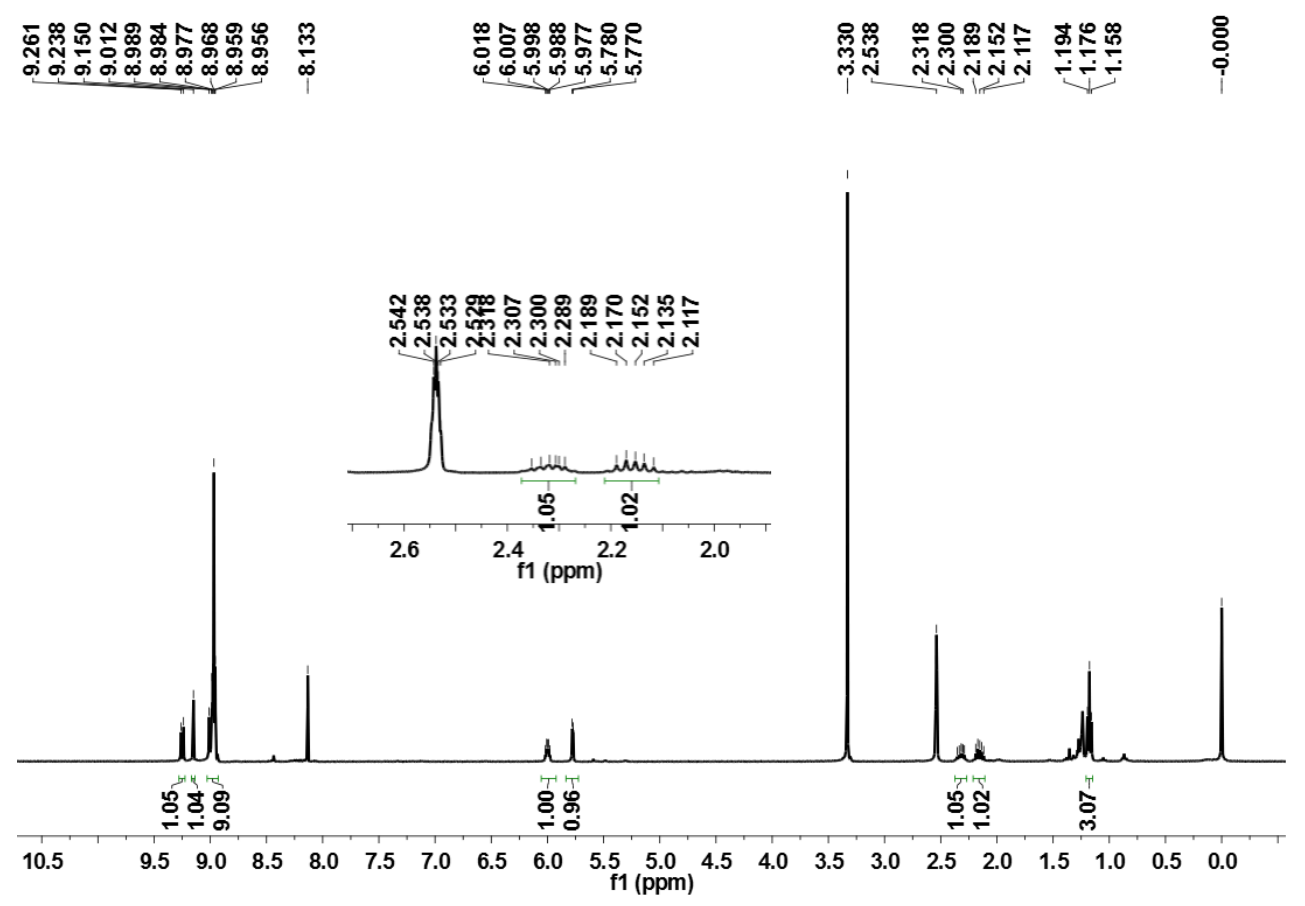




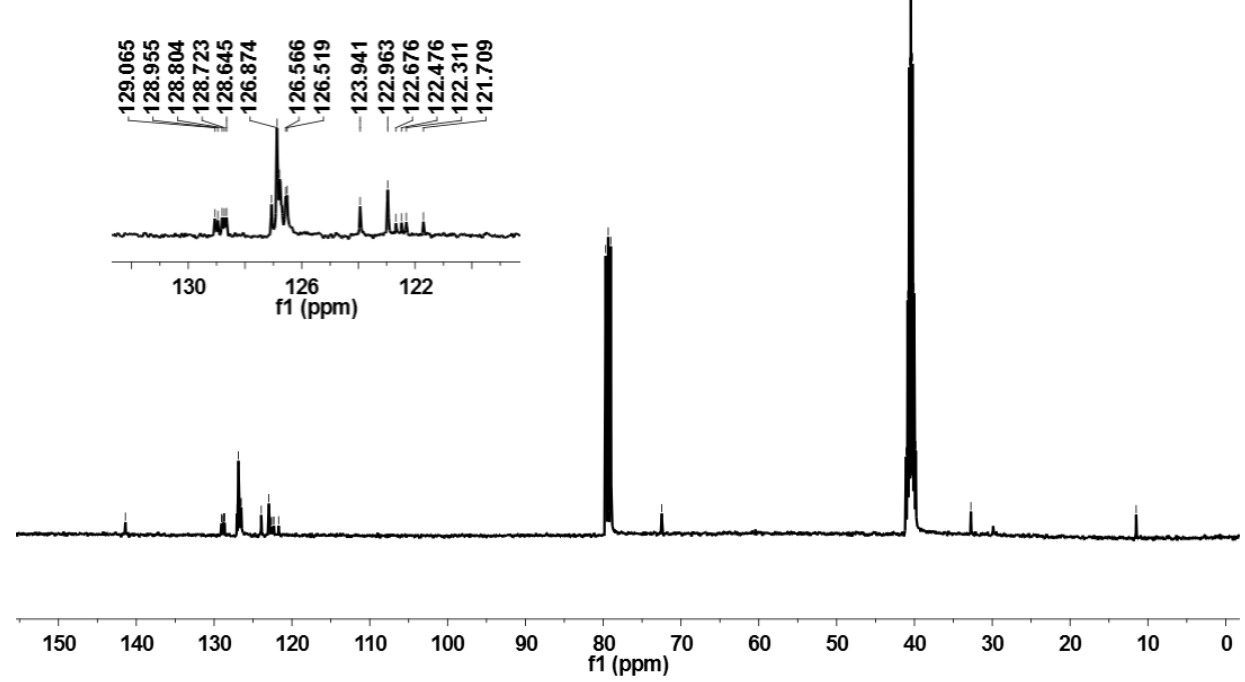

\subsection{NMR and HPLC of the secondary alcohols obtained with CCOF-2/Ti.}<smiles>CCC(O)c1ccccc1</smiles>

(S)-1-phenylpropan-1-ol: Optical purity is determined by HPLC with a ChiralCel OD-H column: $25{ }^{\circ} \mathrm{C}$; hexane $/ \mathrm{PrOH}=98 / 2$; flow rate $=1.0 \mathrm{~mL} / \mathrm{min}$; $\mathrm{t}_{\text {major }}$ $=17.08 \mathrm{~min}, \mathrm{t}_{\mathrm{minor}}=14.67 \mathrm{~min}$; ee $=85 \%$.

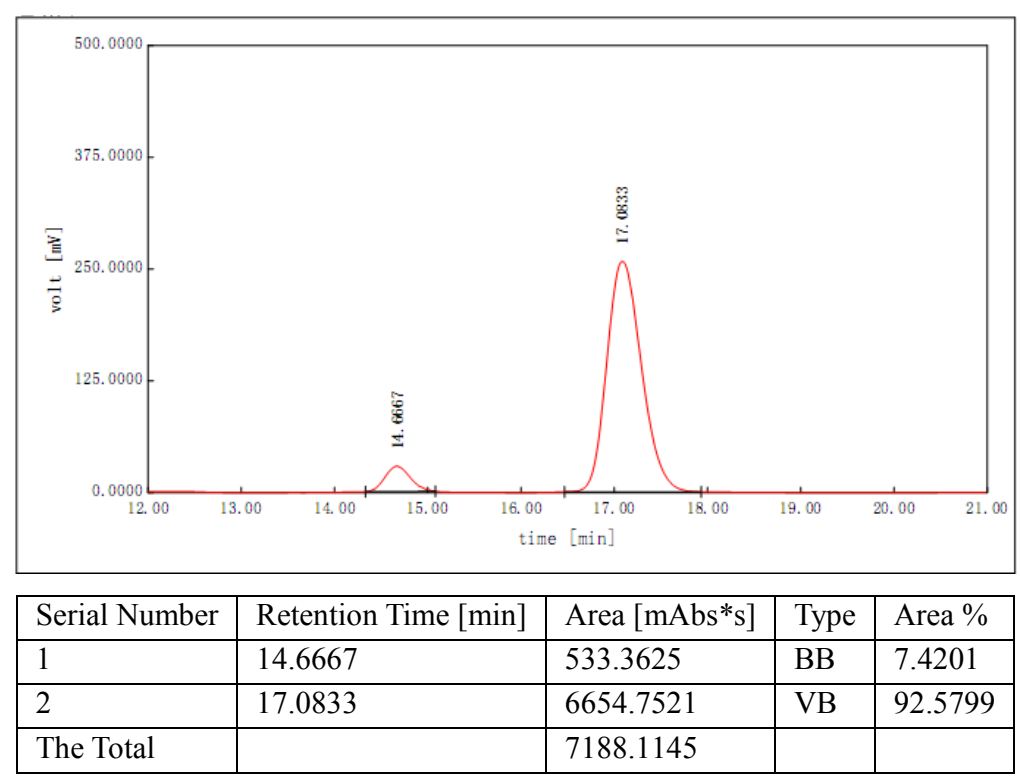<smiles>CC[C@H](O)c1ccccc1</smiles>

(R)-1-phenylpropan-1-ol: Optical purity is determined by HPLC with a ChiralCel OD-H column: $25{ }^{\circ} \mathrm{C}$; hexane $/ \mathrm{PrOH}=98 / 2$; flow rate $=1.0 \mathrm{~mL} / \mathrm{min}$; $\mathrm{t}_{\text {major }}=14.87 \mathrm{~min}, \mathrm{t}_{\text {minor }}=17.68 \mathrm{~min}$; ee $=85 \%$. 


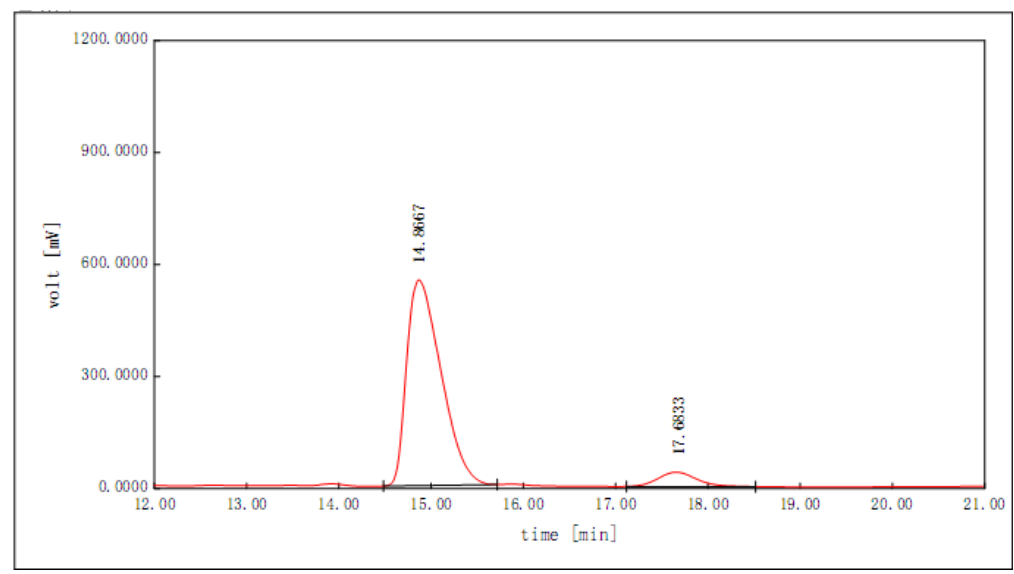

\begin{tabular}{|l|l|l|l|l|}
\hline Serial Number & Retention Time $[\mathrm{min}]$ & Area $[\mathrm{mAbs}$ s] & Type & Area \% \\
\hline 1 & 14.8667 & 14267.1023 & BB & 92.6665 \\
\hline 2 & 17.6833 & 1129.0834 & BB & 7.3335 \\
\hline The Total & & 15396.1857 & & \\
\hline
\end{tabular}

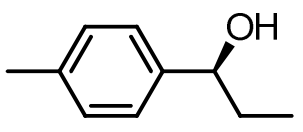

(S)-1-(p-tolyl)propan-1-ol: Optical purity is determined by HPLC with a ChiralCel $\mathrm{AD}-\mathrm{H}$ column: $25^{\circ} \mathrm{C}$; hexane $/ \mathrm{PrOH}=98 / 2$; flow rate $=1.0 \mathrm{~mL} / \mathrm{min}$; $\mathrm{t}_{\text {major }}=17.27 \mathrm{~min}, \mathrm{t}_{\text {minor }}=15.00 \mathrm{~min}$; ee $=88 \%$.

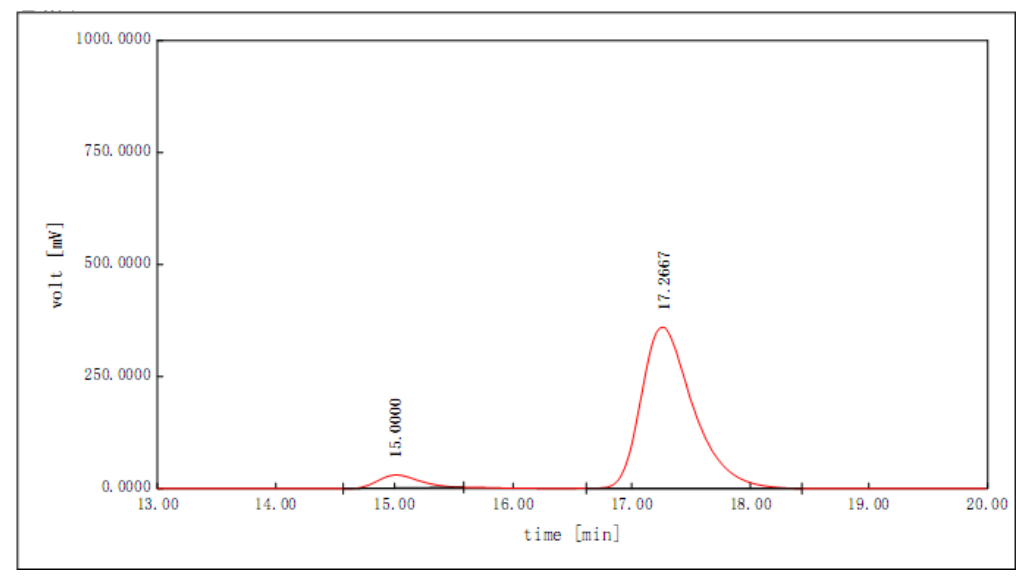

\begin{tabular}{|l|l|l|l|l|}
\hline Serial Number & Retention Time $[\mathrm{min}]$ & Area $[\mathrm{mAbs}$ s] & Type & Area \% \\
\hline 1 & 15.0000 & 709.4993 & BB & 6.0783 \\
\hline 2 & 17.2667 & 10963.0842 & BB & 93.9217 \\
\hline The Total & & 11672.5834 & & \\
\hline
\end{tabular}<smiles>CCC(O)c1ccc(Cl)cc1</smiles>

(S)-1-(4-chlorophenyl)propan-1-ol: Optical purity is determined by HPLC with a ChiralCel OD-H column: $25{ }^{\circ} \mathrm{C}$; hexane/iPrOH $=98 / 2$; flow rate $=1.0 \mathrm{~mL} / \mathrm{min} ; \mathrm{t}_{\text {major }}=12.83 \mathrm{~min}, \mathrm{t}_{\text {minor }}=14.40 \mathrm{~min}$; ee $=86 \%$. 


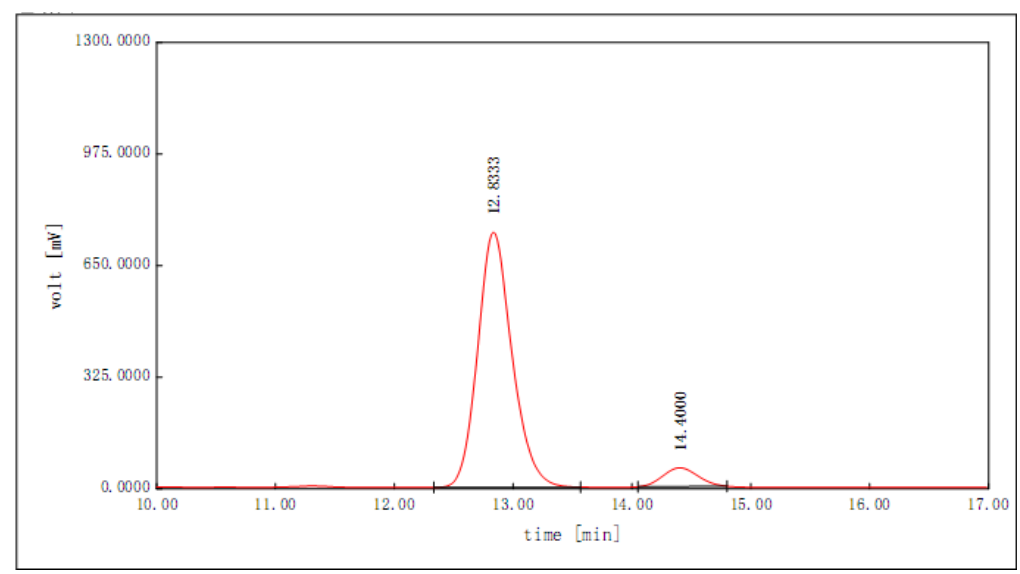

\begin{tabular}{|l|l|l|l|l|}
\hline Serial Number & Retention Time [min] & Area [mAbs*s] & Type & Area \% \\
\hline 1 & 12.8333 & 14910.5929 & BB & 93.0627 \\
\hline 2 & 14.4000 & 1111.4928 & BB & 6.9373 \\
\hline The Total & & 16022.0857 & & \\
\hline
\end{tabular}<smiles>CCC(O)c1ccc2ccccc2c1</smiles>

(S)-1-(naphthalen-2-yl)propan-1-ol: Optical purity is determined by HPLC with a ChiralCel OD-H column: $25{ }^{\circ} \mathrm{C}$; hexane/iPrOH $=90 / 10$; flow rate $=0.5$ $\mathrm{mL} / \mathrm{min} ; \mathrm{t}_{\text {major }}=19.73 \mathrm{~min}, \mathrm{t}_{\mathrm{minor}}=21.62 \mathrm{~min} ; \mathrm{ee}=95 \%$.

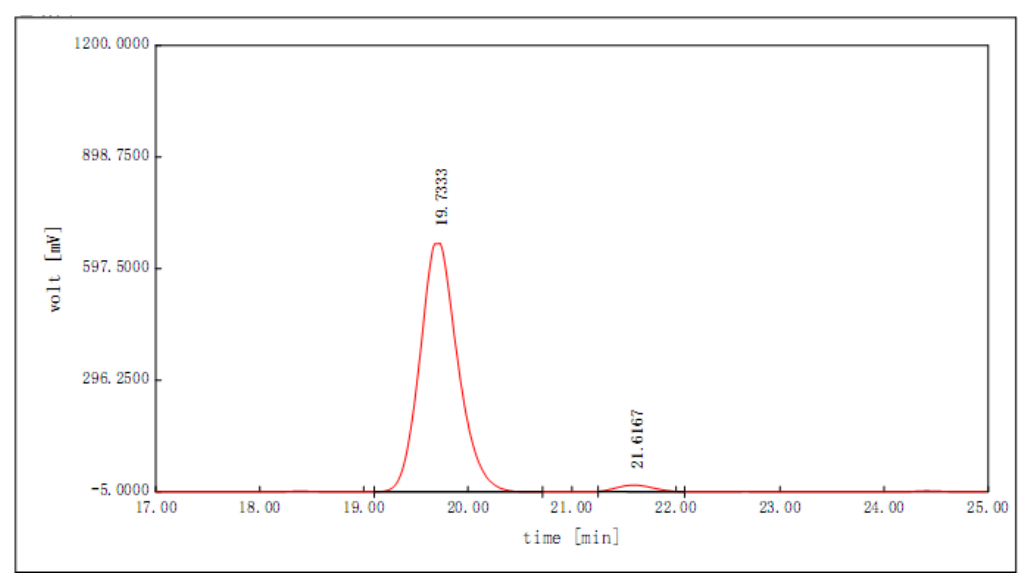

\begin{tabular}{|l|l|l|l|l|}
\hline Serial Number & Retention Time $[\mathrm{min}]$ & Area $[\mathrm{mAbs*}$ s] & Type & Area \% \\
\hline 1 & 19.7333 & 17098.4475 & BB & 97.4513 \\
\hline 2 & 21.6167 & 447.1907 & BB & 2.5487 \\
\hline The Total & & 17545.6382 & & \\
\hline
\end{tabular}

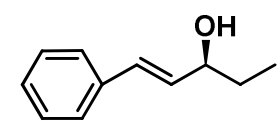

$\boldsymbol{S}$-(E)-1-phenylpent-1-en-3-ol: Optical purity is determined by HPLC with a ChiralCel OD-H column: $25{ }^{\circ} \mathrm{C}$; hexane/iPrOH $=95 / 5$; flow rate $=1.0$ $\mathrm{mL} / \mathrm{min} ; \mathrm{t}_{\text {major }}=23.42 \mathrm{~min}, \mathrm{t}_{\text {minor }}=13.50 \mathrm{~min} ; \mathrm{ee}=85 \%$. 


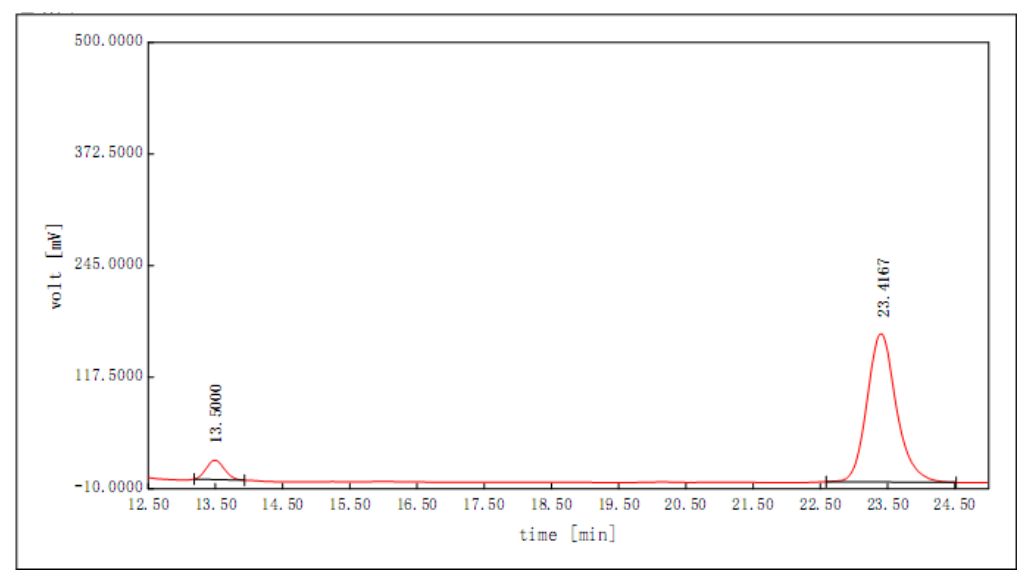

\begin{tabular}{|l|l|l|l|l|}
\hline Serial Number & Retention Time [min] & Area [mAbs*s] & Type & Area \% \\
\hline 1 & 13.5000 & 412.1645 & BB & 7.4271 \\
\hline 2 & 23.4167 & 5137.2841 & BB & 92.5729 \\
\hline The Total & & 5549.4486 & & \\
\hline
\end{tabular}<smiles>CCC(O)c1cc2cccc3ccc4cccc1c4c32</smiles>

(S)-1-(pyren-4-yl)propan-1-ol: Optical purity is determined by HPLC with a ChiralCel OD-H column: $25{ }^{\circ} \mathrm{C}$; hexane/iPrOH $=90 / 10$; flow rate $=1.0$ $\mathrm{mL} / \mathrm{min} ; \mathrm{t}_{\text {major }}=14.73 \mathrm{~min}, \mathrm{t}_{\text {minor }}=17.15 \mathrm{~min} ; \mathrm{ee}=74 \%$.

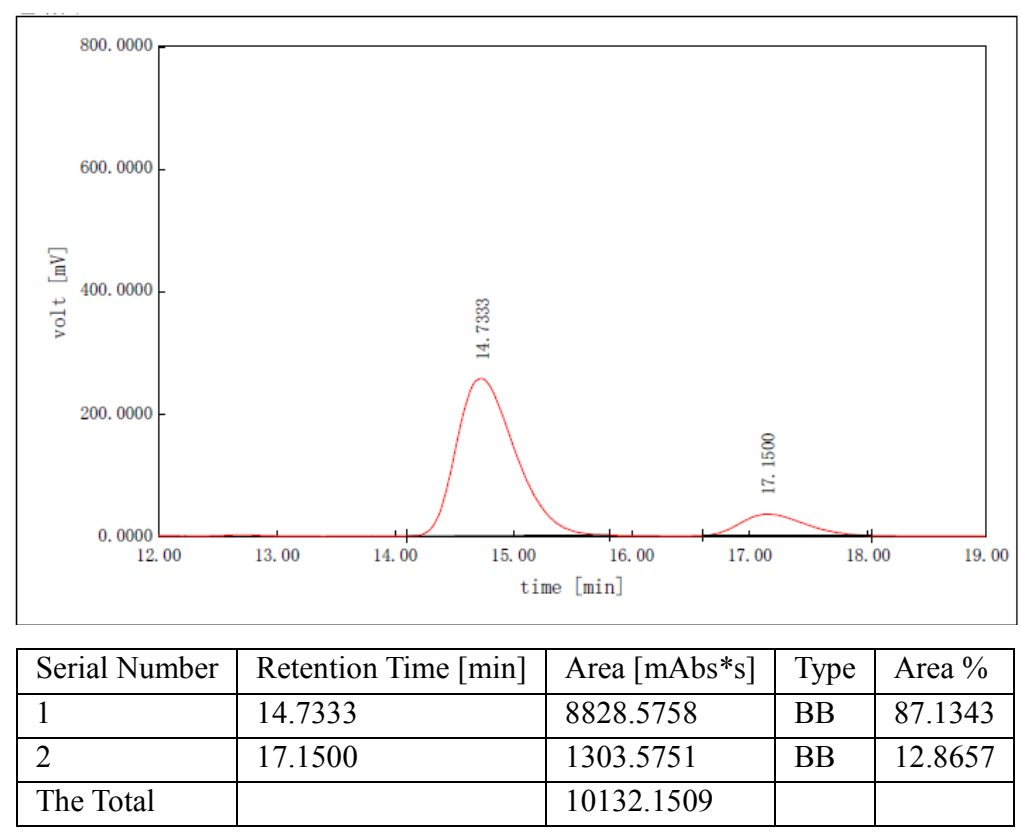

\subsection{HPLC of the secondary alcohols obtained with homogeneous TADDOL/Ti}

The ee values of the alcohol products derived from $\mathrm{C}_{6} \mathrm{H}_{5} \mathrm{CH}=\mathrm{CHO}, \mathrm{C}_{6} \mathrm{H}_{5} \mathrm{CHO}$ are used the reported date in Bull. Chem. Soc. Jpn. 2014, 87, 435 (Wang, X. et al.) 
(S)-1-phenylpropan-1-ol: Optical purity is determined by HPLC with a ChiralCel OD-H column: $25{ }^{\circ} \mathrm{C}$; hexane $/ \mathrm{PrOH}=98 / 2$; flow rate $=1.0 \mathrm{~mL} / \mathrm{min}$; $\mathrm{t}_{\text {major }}=16.83 \mathrm{~min}, \mathrm{t}_{\mathrm{minor}}=14.35 \mathrm{~min} ; \mathrm{ee}=90 \%$.

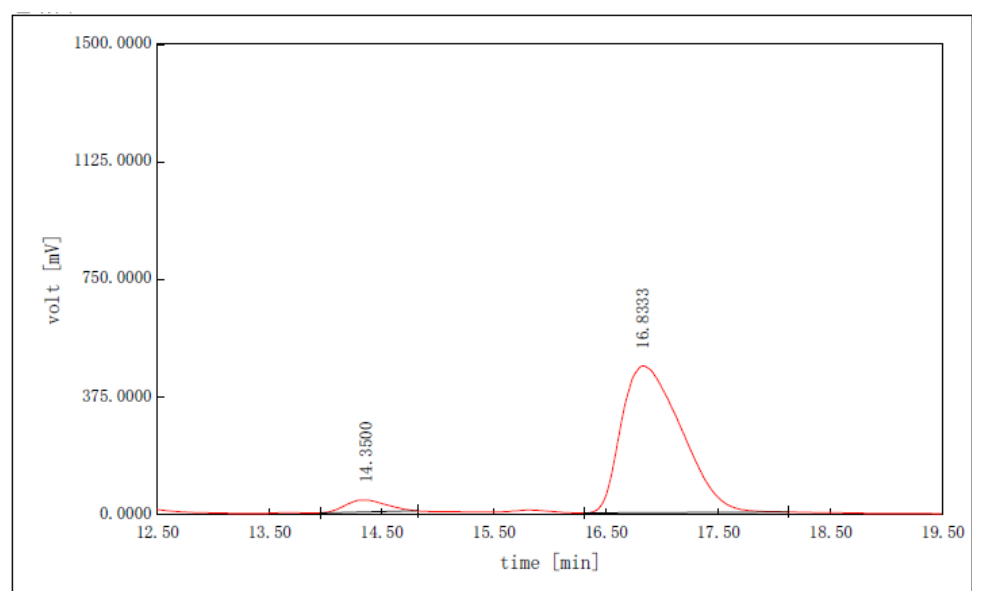

\begin{tabular}{|l|l|l|l|l|}
\hline Serial Number & Retention Time [min] & Area $[\mathrm{mAbs}$ s] & Type & Area \% \\
\hline 1 & 14.3500 & 968.7061 & BB & 5.2834 \\
\hline 2 & 16.8333 & 17366.3447 & VB & 94.7166 \\
\hline The Total & & 18335.0507 & & \\
\hline
\end{tabular}
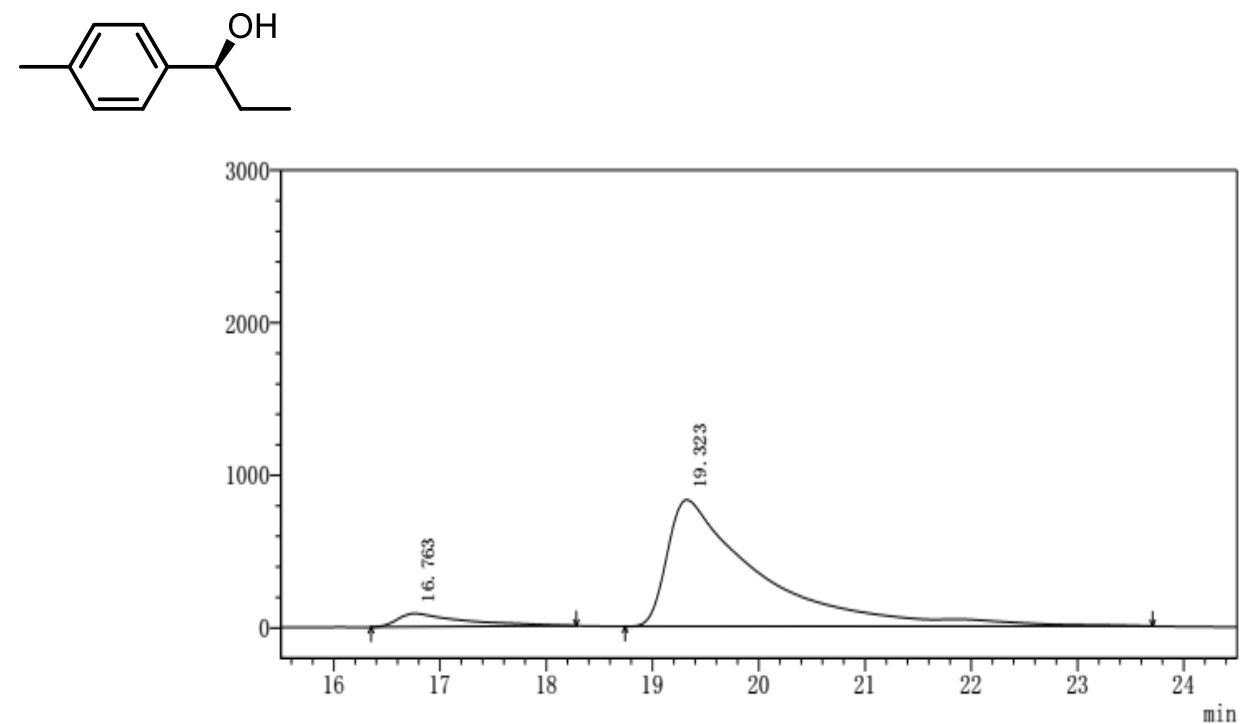

\begin{tabular}{|l|l|l|l|l|}
\hline Serial Number & Retention Time [min] & Area $[\mathrm{mAbs*}$ ] & Type & Area \% \\
\hline 1 & 16.763 & 3768920 & BB & 7.008 \\
\hline 2 & 19.323 & 50014022 & BB & 92.992 \\
\hline The Total & & 53782942 & & \\
\hline
\end{tabular}<smiles>CCC(O)c1ccc(Cl)cc1</smiles> 


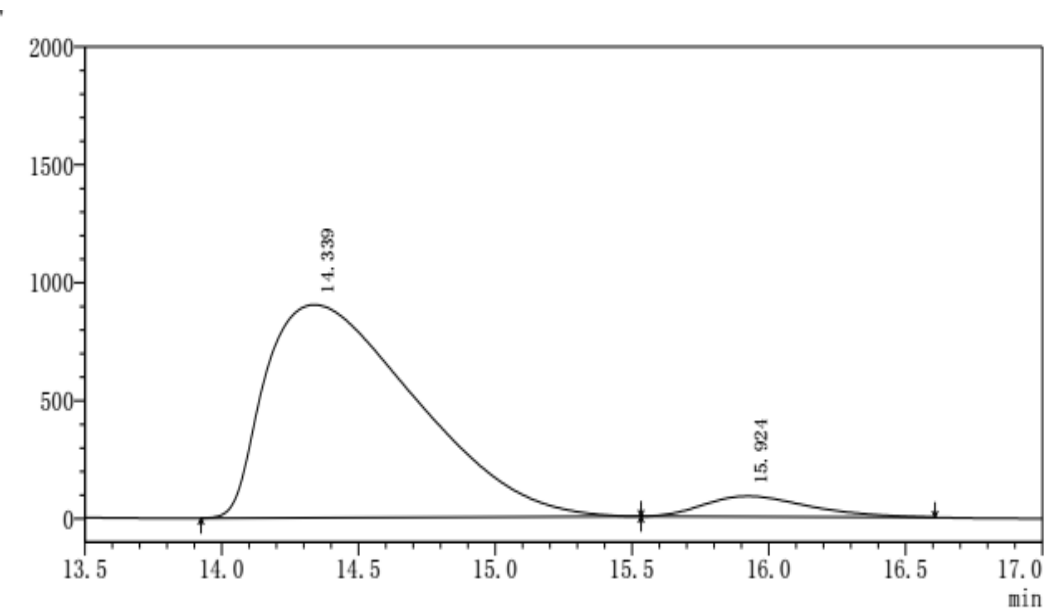

\begin{tabular}{|l|l|l|l|l|}
\hline Serial Number & Retention Time $[\mathrm{min}]$ & Area $[\mathrm{mAbs}$ s] & Type & Area \% \\
\hline 1 & 14.339 & 34585277 & BB & 93.669 \\
\hline 2 & 15.924 & 2337495 & BB & 6.331 \\
\hline The Total & & 36922772 & & \\
\hline
\end{tabular}<smiles>CCC(O)c1ccc2ccccc2c1</smiles>

(S)-1-(naphthalen-2-yl)propan-1-ol: Optical purity is determined by HPLC with a ChiralCel OD-H column: $25^{\circ} \mathrm{C}$; hexane/iPrOH $=90 / 10$; flow rate $=0.5$ $\mathrm{mL} / \mathrm{min} ; \mathrm{t}_{\text {major }}=19.28 \mathrm{~min}, \mathrm{t}_{\text {minor }}=21.62 \mathrm{~min} ; \mathrm{ee}=93 \%$.

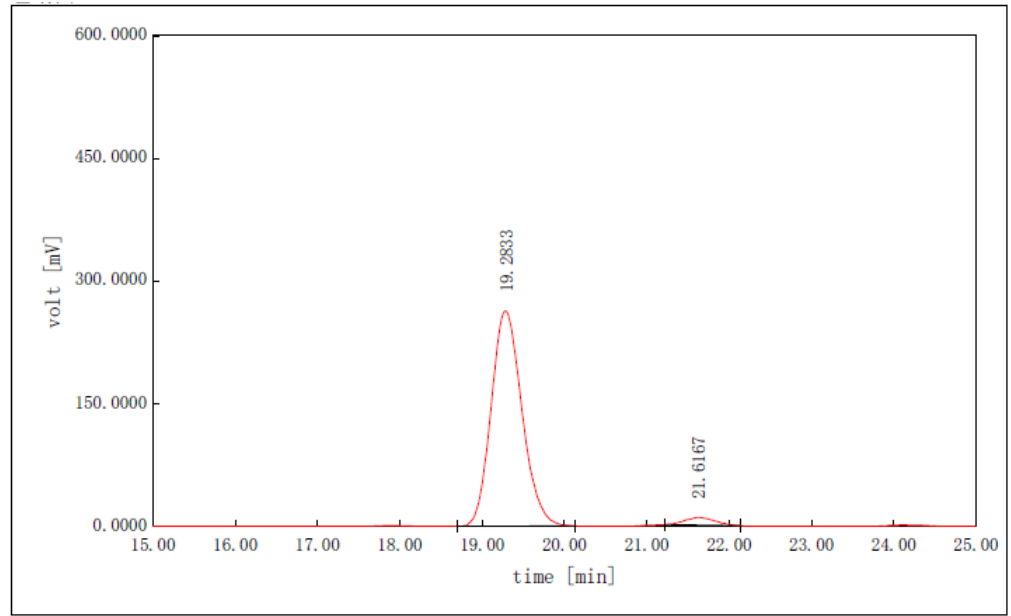

\begin{tabular}{|l|l|l|l|l|}
\hline Serial Number & Retention Time $[\mathrm{min}]$ & Area $[\mathrm{mAbs}$ *s] & Type & Area \% \\
\hline 1 & 19.2833 & 6851.2618 & BB & 96.6882 \\
\hline 2 & 21.6167 & 234.6735 & BB & 3.3118 \\
\hline The Total & & 7085.9353 & & \\
\hline
\end{tabular}


<smiles>CCC(O)c1cc2cccc3ccc4cccc1c4c32</smiles>

(S)-1-(pyren-4-yl)propan-1-ol: Optical purity is determined by HPLC with a ChiralCel OD-H column: $25{ }^{\circ} \mathrm{C}$; hexane/iPrOH $=90 / 10$; flow rate $=1.0$ $\mathrm{mL} / \mathrm{min} ; \mathrm{t}_{\text {major }}=14.30 \mathrm{~min}, \mathrm{t}_{\text {minor }}=16.65 \mathrm{~min} ; \mathrm{ee}=78 \%$.

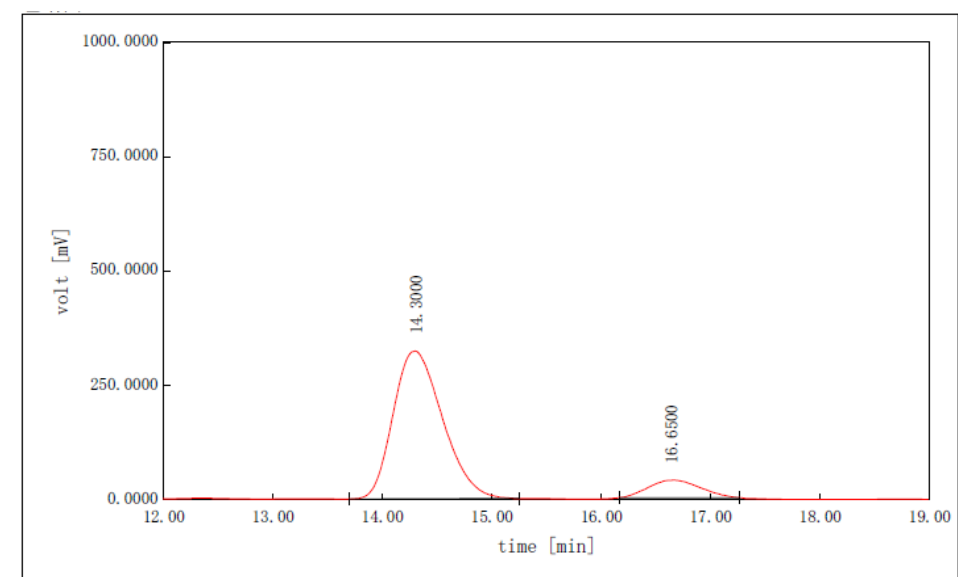

\begin{tabular}{|l|l|l|l|l|}
\hline Serial Number & Retention Time $[\mathrm{min}]$ & Area $[\mathrm{mAbs}$ *s] & Type & Area \% \\
\hline 1 & 14.3000 & 10117.0089 & BB & 88.9408 \\
\hline 2 & 16.6500 & 1257.9823 & BB & 11.0592 \\
\hline The Total & & 11374.9912 & & \\
\hline
\end{tabular}

\subsection{Catalytic recycling results by $\mathrm{CCOF}-1 / \mathrm{Ti}$}

: (S)-1-phenylpropan-1-ol: Optical purity is determined by HPLC with a ChiralCel OD-H column: $25^{\circ} \mathrm{C}$; hexane $/ \mathrm{PrOH}=98 / 2$; flow rate $=1.0 \mathrm{~mL} / \mathrm{min}$.

\section{The $1^{\text {st }}$ run}

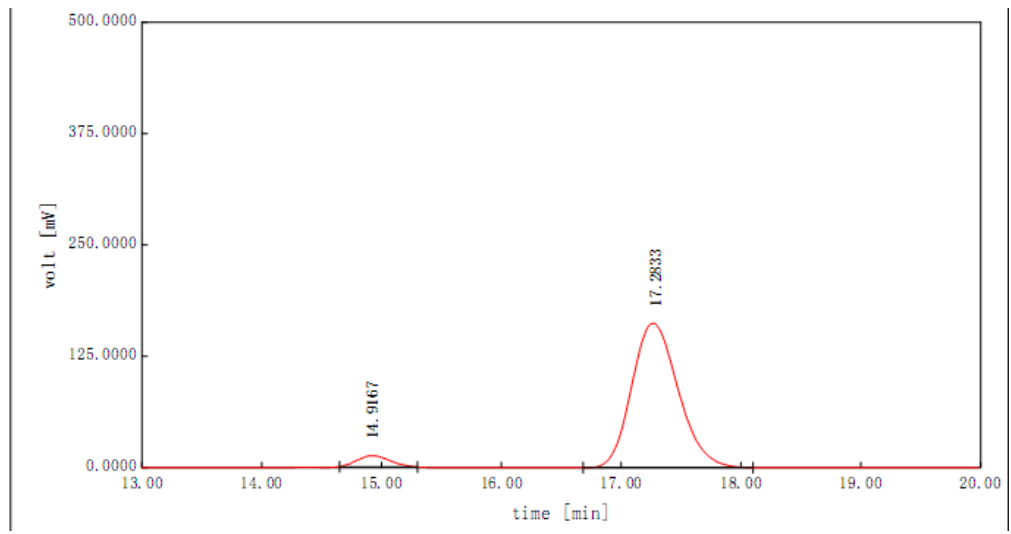

\begin{tabular}{|l|l|l|l|l|}
\hline Serial Number & Retention Time [min] & Area [mAbs*s] & Type & Area \% \\
\hline 1 & 14.9167 & 237.1649 & BB & 5.1259 \\
\hline 2 & 17.2833 & 4389.6497 & BB & 94.8741 \\
\hline The Total & & 4626.8146 & & \\
\hline
\end{tabular}




\section{The $2^{\text {nd }}$ run}

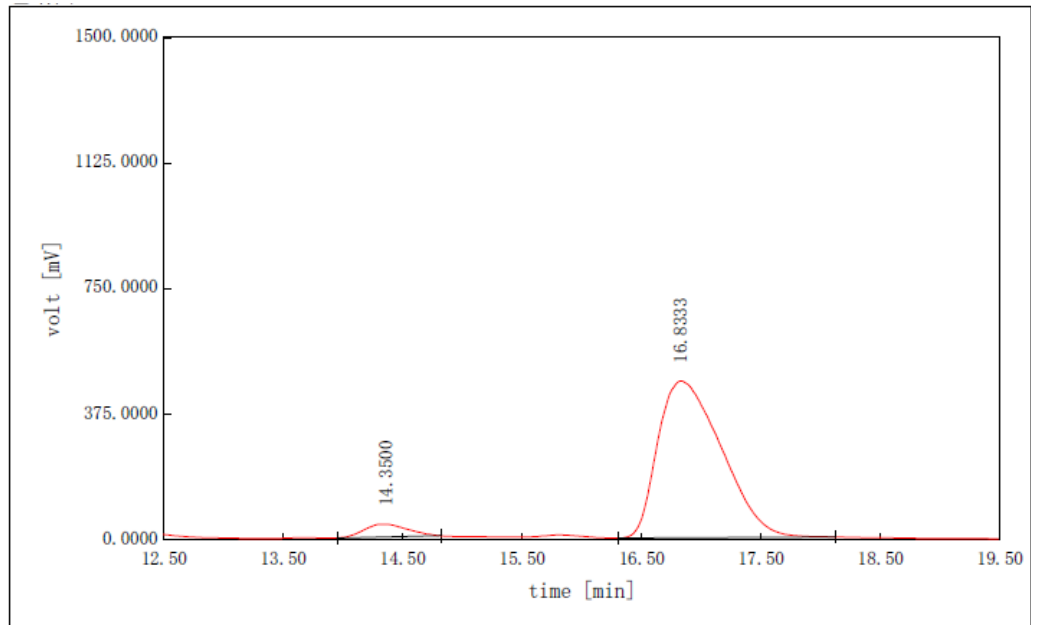

\begin{tabular}{|l|l|l|l|l|}
\hline Serial Number & Retention Time $[\mathrm{min}]$ & Area $[\mathrm{mAbs} * \mathrm{~s}]$ & Type & Area \% \\
\hline 1 & 14.3500 & 961.3801 & BB & 5.2434 \\
\hline 2 & 16.8333 & 17373.6706 & VB & 94.7566 \\
\hline The Total & & 18335.0507 & & \\
\hline
\end{tabular}

The $3^{\text {rd }}$ run

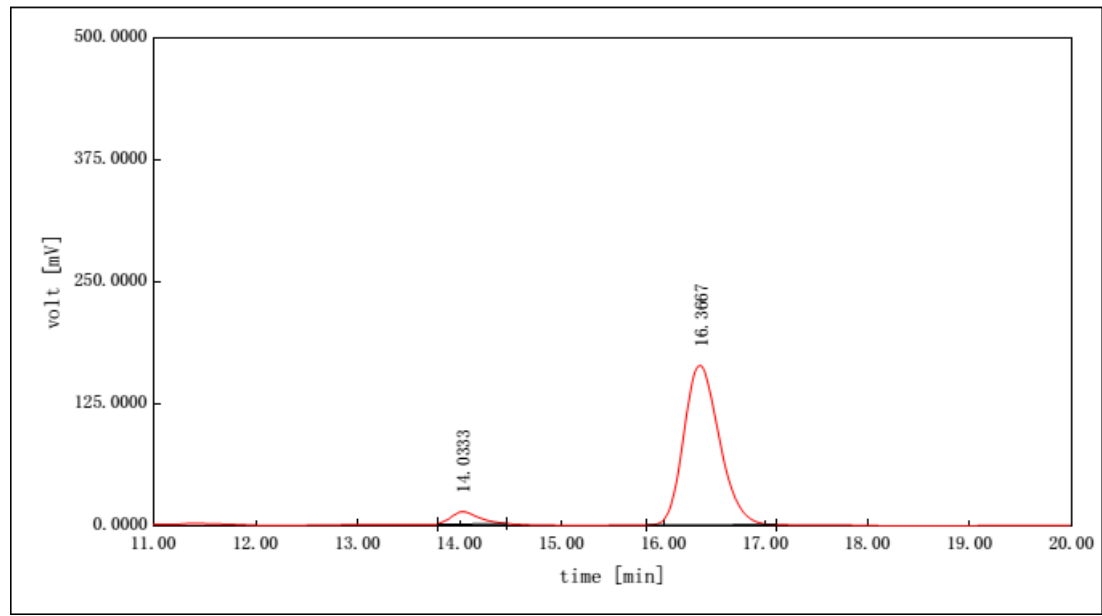

\begin{tabular}{|l|l|l|l|l|}
\hline Serial Number & Retention Time [min] & Area [mAbs*s] & Type & Area \% \\
\hline 1 & 14.0333 & 241.7211 & BB & 5.7494 \\
\hline 2 & 16.3667 & 3962.5339 & BB & 94.2506 \\
\hline The Total & & 4204.2550 & & \\
\hline
\end{tabular}

The $4^{\text {th }}$ run 


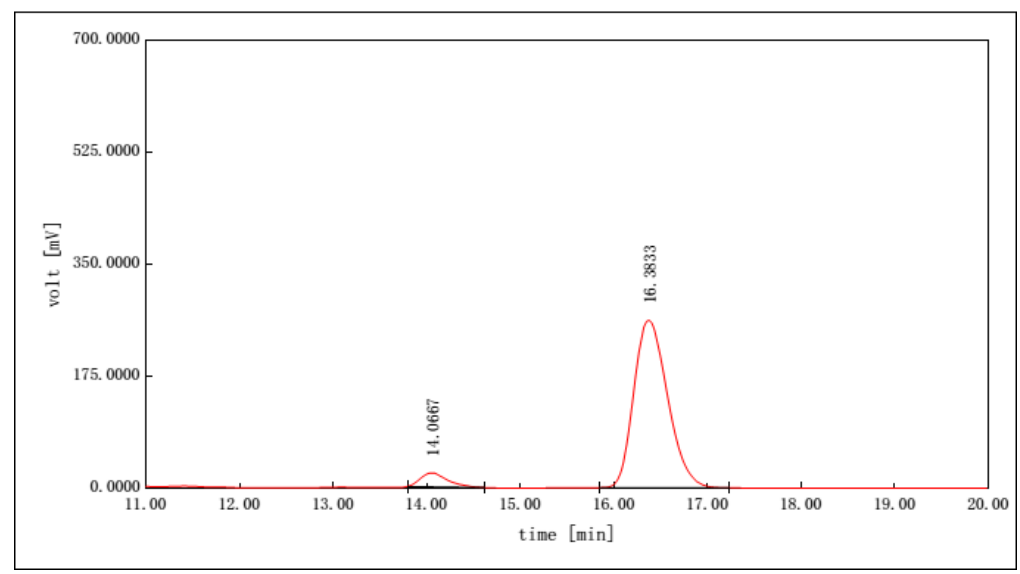

\begin{tabular}{|l|l|l|l|l|}
\hline Serial Number & Retention Time [min] & Area [mAbs*s] & Type & Area \% \\
\hline 1 & 14.0667 & 432.8235 & BB & 6.1663 \\
\hline 2 & 16.3833 & 6586.3377 & BB & 93.8337 \\
\hline The Total & & 7019.1613 & & \\
\hline
\end{tabular}

The $5^{\text {th }}$ run

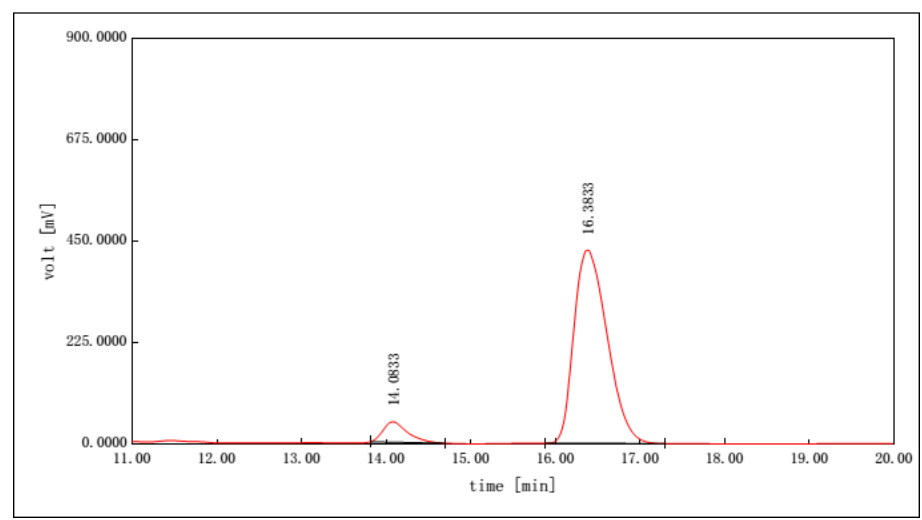

\begin{tabular}{|l|l|l|l|l|}
\hline Serial Number & Retention Time [min] & Area [mAbs*s] & Type & Area \% \\
\hline 1 & 14.0833 & 786.6278 & BB & 6.2457 \\
\hline 2 & 16.3833 & 11808.0913 & BB & 93.7543 \\
\hline The Total & & 12594.7191 & & \\
\hline
\end{tabular}

\subsection{Catalytic recycling results by CCOF-2/Ti}<smiles>CCC(O)c1ccccc1</smiles>

(S)-1-phenylpropan-1-ol: Optical purity is determined by HPLC with a ChiralCel OD-H column: $25^{\circ} \mathrm{C}$; hexane $/ \mathrm{PrOH}=98 / 2$; flow rate $=1.0 \mathrm{~mL} / \mathrm{min}$.

\section{The $1^{\text {st }}$ run}




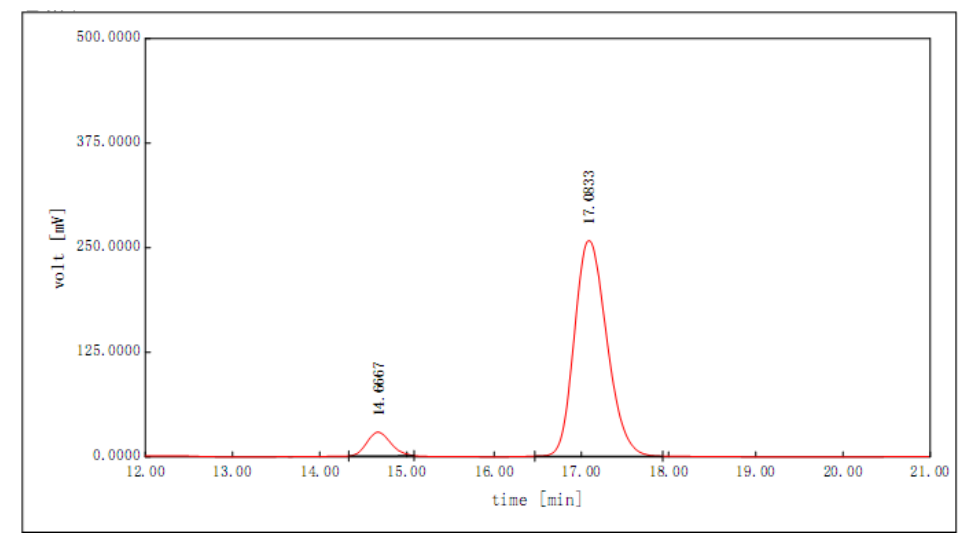

\begin{tabular}{|l|l|l|l|l|}
\hline Serial Number & Retention Time [min] & Area $[\mathrm{mAbs} * \mathrm{~s}]$ & Type & Area \% \\
\hline 1 & 14.6667 & 533.3625 & BB & 7.4201 \\
\hline 2 & 17.0833 & 6654.7521 & VB & 92.5799 \\
\hline The Total & & 7188.1145 & & \\
\hline
\end{tabular}

\section{The $2^{\text {nd }}$ run}

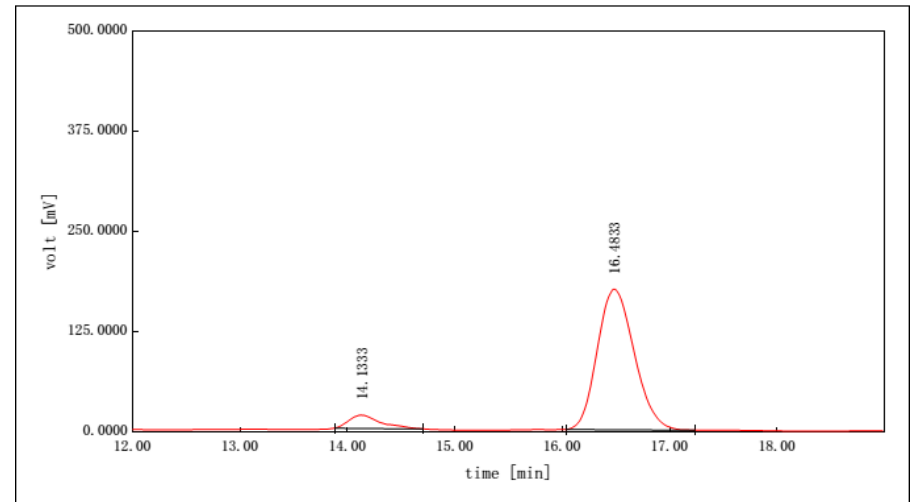

\begin{tabular}{|l|l|l|l|l|}
\hline Serial Number & Retention Time [min] & Area [mAbs*s] & Type & Area \% \\
\hline 1 & 14.1333 & 344.6776 & BB & 7.5981 \\
\hline 2 & 16.4833 & 4191.6951 & BB & 92.4019 \\
\hline The Total & & 4536.3727 & & \\
\hline
\end{tabular}

The $3^{\text {rd }}$ run

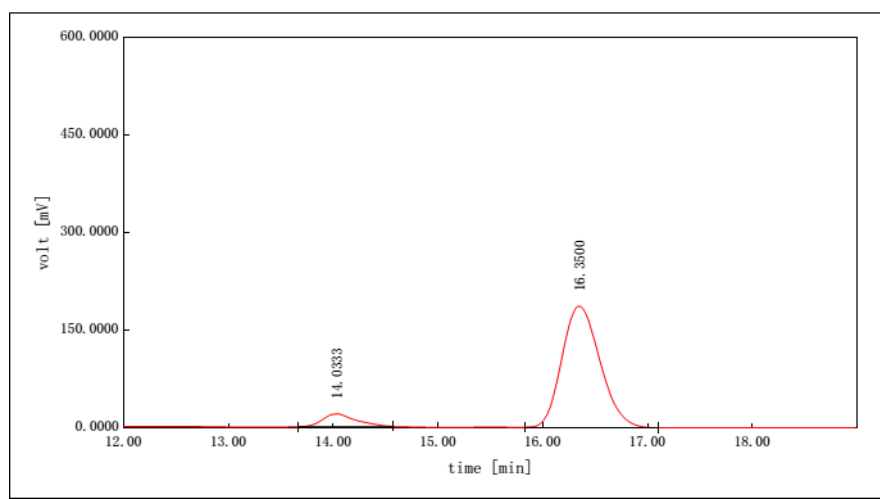

\begin{tabular}{|l|l|l|l|l|}
\hline Serial Number & Retention Time $[\mathrm{min}]$ & Area $[\mathrm{mAbs*}$ ) & Type & Area \% \\
\hline 1 & 14.0333 & 432.8188 & BB & 8.4019 \\
\hline 2 & 16.3500 & 4718.6250 & BB & 91.5981 \\
\hline The Total & & 5151.4438 & & \\
\hline
\end{tabular}


The $4^{\text {th }}$ run

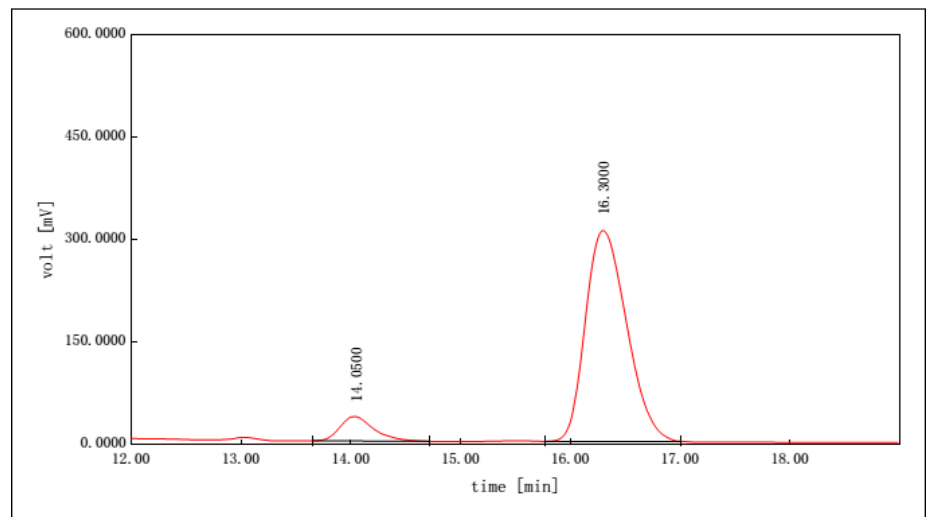

\begin{tabular}{|l|l|l|l|l|}
\hline Serial Number & Retention Time $[\mathrm{min}]$ & Area $[\mathrm{mAbs*}$ s] & Type & Area \% \\
\hline 1 & 14.0500 & 764.4358 & BB & 8.6677 \\
\hline 2 & 16.3000 & 8054.8893 & BB & 91.3323 \\
\hline The Total & & 8819.3251 & & \\
\hline
\end{tabular}

\section{The $5^{\text {th }}$ run}

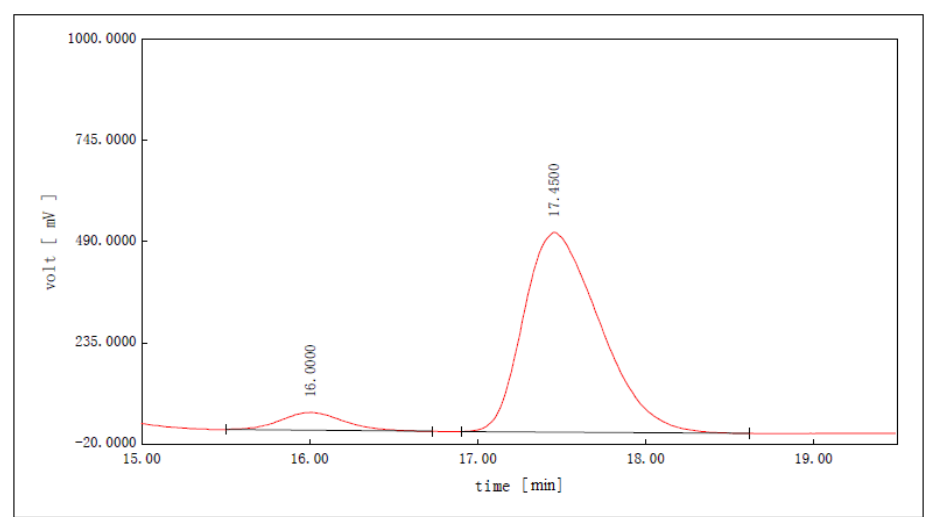

\begin{tabular}{|l|l|l|l|l|}
\hline Serial Number & Retention Time [min] & Area [mAbs*s] & Type & Area \% \\
\hline 1 & 15.591 & 109828 & BB & 8.715 \\
\hline 2 & 17.382 & 1150384 & BB & 91.285 \\
\hline The Total & & 1260212 & & \\
\hline
\end{tabular}


16 Figure S15. Calculated space filling model of different aromatic aldehydes.

The space-filling models were calculated by Materials Studio (7.0), and were fully optimized using MS Forcite molecular dynamics module (universal force fields, Ewald summations) method.
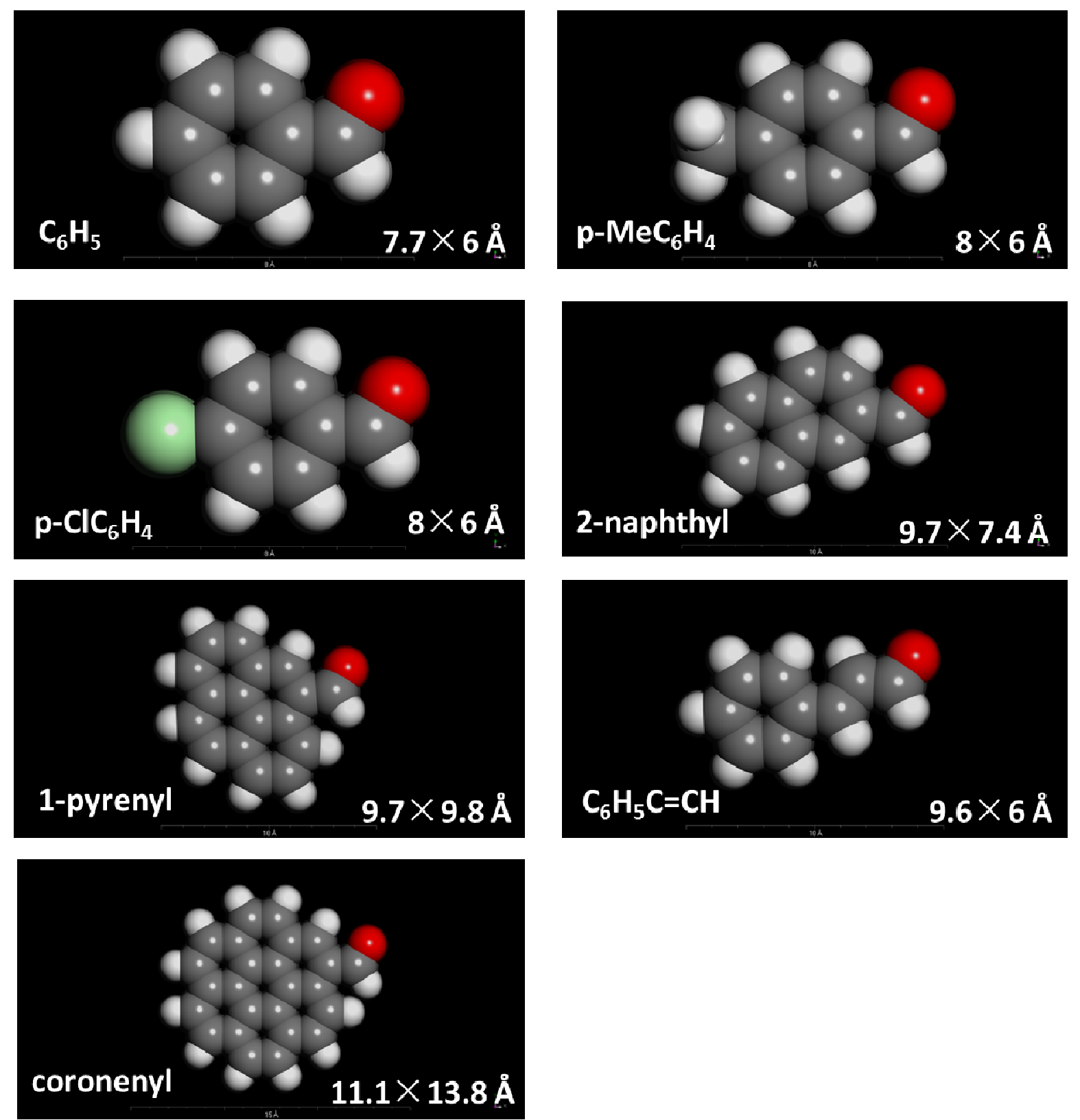